UNIVERSIDAD POLITÉCNICA DE MADRID

Escuela Técnica Superior de Ingenieros Industriales

\title{
Numerical Methods for the simulation of high velocity impact
}

DOCTORAL THESIS

Author:

Daniel DEL POZO

Ingeniero Industrial

November 26, 2018 



\section{DEPARTAMENTO DE INGENIERÍA MECÁNICA}

\section{Numerical Methods for the simulation of high velocity impact}

DOCTORAL THESIS

Author:

Daniel DEL Pozo

Ingeniero Industrial

Director:

Ignacio Romero Olleros

Doctor Ingeniero Industrial

November 26, 2018
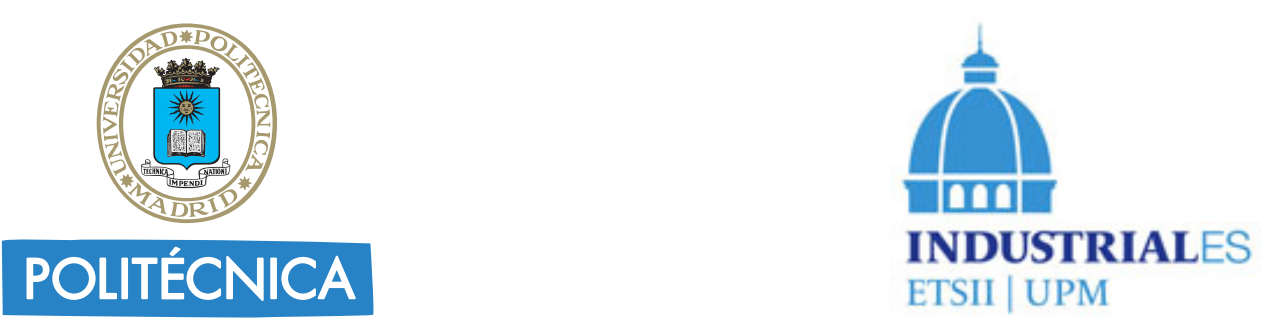

Tribunal nombrado por el Magfco. y Excmo. Sr. Rector de la Universidad Politécnica de Madrid, el día . . . . . . de . . . . . . de 2018.

- Presidente: D

- Vocal: D.

- Vocal: D.

- Vocal: D.

- Secretario: D.

Realizado el acto de defensa y lectura de la tesis el día. . . . . . de . . . . . . de 2018, en la Escuela Técnica Superior de Ingenieros Industriales, de la Universidad Politécnica de Madrid.

Calificación: 

"There are men who struggle for a day and they are good. There are men who struggle for a year and they are better. There are men who struggle many years, and they are better still. But there are those who struggle all their lives: These are the indispensable ones."

Bertolt Bretch 



\title{
Universidad Politécnica de Madrid
}

\author{
Abstract \\ Escuela Técnica Superior de Ingenieros Industriales \\ Imdea Materials
}

\section{Numerical Methods for the simulation of high velocity impact}

by Daniel DEL POZO

This work studies the phenomena of high speed impact in aeronautics and its consequences from a computational perspective. We consider within the wide range of impacting bodies only those considered to have low strength compared to the target, namely ice and bird. This choice implies that the discretised bodies will undergo large distortions during impact as well as the mesh. Meshless approximation are very well suited for this kind of impact problems and are adopted in this dissertation. Also a contact algorithm has been developed to perform meshless and FEM interaction. Due to the wide variety of impacting velocities and the possible material dependence to this variation, a class of visco elasto-plastic constitutive models based on the notion of Duvaut-Lions viscoplasticity have been developed. This constitutive model ensures favorable dissipation and gives a wide variety of different behaviours for different kinematic convex potentials. All of this will be applied finally to a more complex case assessing the damage produced by the impact at different conditions.

Este documento estudia el fenómeno del impacto a alta velocidad en la aeronáutica y sus consecuencias desde la perspectiva de la mecánica computacional. Hemos considerado dado la gran variedad de objetos impactantes, solo aquellos que son considerados de baja resistencia con respecto a la estructura que es impactada, por ejemplo hielo y pájaro. Esta elección implica que los cuerpos discretizados sufrirán importantes distorsiones durante el impacto, lo que implica también distorsión del mallado. Los métodos sin malla son ideales para este tipo de problemas de impacto y por eso serán utilizados en este trabajo. También se ha desarrollado un algoritmo de contacto para la interacción de los métodos sin malla y MEF. Dado la variedad de velocidades de impacto y la posible dependencia material a esta variación, se ha desarrollado una clase de modelos constitutivos visco-elasto-plásticos basados en la formulación de Duvaut-Lions. El modelo desarrollado asegura una correcta disipación y da una gran variedad de comportamientos distintos en función de la elección del potencial cinemático que ha de ser convexo. Todo esto se aplicará finalemente a un caso más complejo evaluando el daño ocasionado por el impacto en diferentes condiciones. 



\section{Acknowledgements}

Este trabajo concluye una etapa de 4 años que me ha permitido crecer como investigador y persona pero esto no hubiera sido posible sin una serie de personas a las que le quiero dedicar un agradecimiento especial.

En primer lugar quiero dar las gracias a mi director de tesis Ignacio Romero, por toda la ayuda y apoyo durante este periodo, por su coherencia y actitud que propician que el trabajo sea bueno y que uno se esfuerce por dar lo mejor y por último por su capacidad y sabiduría siempre guardando esa humildad que le caracteriza, convirtiéndose en una referencia para mi.

También quiero agradecer a mis compañeros y amigos Eva, con su infinita bondad, y David. Con ellos he compartido momentos de alegría y complicidad, estando siempre dispuestos a ayudarme.

Por último quería agradecer a mi familia por su educación y amor que me ha enseñado a vivir de una manera coherente siendo el centro de gravedad en mi vida. 



\section{Contents}

Abstract vii

\begin{tabular}{|l|l}
\hline Acknowledgements & ix \\
\hline &
\end{tabular}

\begin{tabular}{llr}
\hline & Introduction & 1
\end{tabular}

1.1 Motivation . . . . . . . . . . . . . . . . . . . 1

1.1 .1 Ice impact . . . . . . . . . . . . . . . . . . . . . 3

1.1 .2 Bird Impact . . . . . . . . . . . . . . . . . . . . . . . . 4

1.2 State of the $\operatorname{art} \ldots \ldots \ldots \ldots \ldots \ldots$

Lagrangian finite element approach . . . . . . . . . . . 6

Arbitrary Lagrangian-Eulerian approach . . . . . . . . . . . . . 8

Smoothed particle hydrodynamics approach . . . . . . . . . 9

Concluding remarks on available spatial discretisations. . . . . 9

1.3 Main lines of work $\ldots \ldots \ldots \ldots$. . . . . . . . . . . 9

1.3 .1 Constitutive models . . . . . . . . . . . . . . . . . . . . 11

1.3.2 Interfaces for material subroutines . . . . . . . . . . . . . . . 11

1.3.3 Spatial discretisation methods . . . . . . . . . . . . . . . . 11

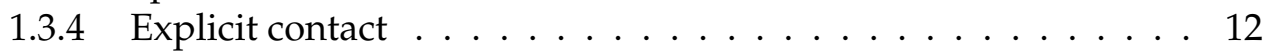

\begin{tabular}{|lll}
\hline 2 & Rate dependent materials & 13
\end{tabular}

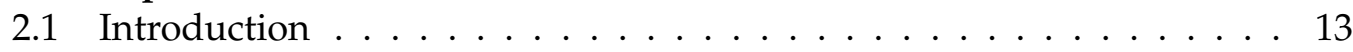

2.2 Plasticity and viscoplasticity models for non-smooth yield criteria . . . 14

$2.2 .1 \quad$ Thermodynamic setting $\ldots \ldots \ldots \ldots \ldots 14$

2.2 .2 Plasticity $\ldots \ldots \ldots \ldots \ldots \ldots$

$2.2 .3 \quad$ Viscoplasticity . . . . . . . . . . . . . . . . . . . . . . . 17

2.3 Integration algorithms for general elasto-visco-plasticity . . . . . . . . 19

2.3 .1 General structure . . . . . . . . . . . . . . . . . . . . . 19

2.3 .2 The abstract rate-independent problem . . . . . . . . . . . . 20

$2.3 .3 \quad$ The projection problem $\ldots \ldots \ldots \ldots$. . . . . . . . . . . 20

$2.3 .4 \quad$ Local stability estimate . . . . . . . . . . . . . . . . . . 22

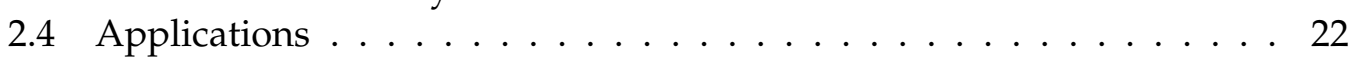

2.4 .1 Tresca's viscoplasticity . . . . . . . . . . . . . . . . 23

2.4 .2 Drucker-Prager's viscoplasticity . . . . . . . . . . . . . . . . . . . 24

$2.4 .3 \quad$ Numerical examples . . . . . . . . . . . . . . . . . . . . 25

Strip footing . . . . . . . . . . . . . . . . . . 25

Traction test . . . . . . . . . . . . . . . . . 27

Perforated plate . . . . . . . . . . . . . . . . . . . 29

Taylor bar . . . . . . . . . . . . . . . . . 30

2.5 Summary of main results . . . . . . . . . . . . . . . . . . . . . 30

2.6 MUESLI $\ldots \ldots \ldots \ldots \ldots \ldots$

2.6 .1 Reliable . . . . . . . . . . . . . . . . . . 32

2.6 .2 Extendable . . . . . . . . . . . . . . . . . . . 33

2.6 .3 Linkable . . . . . . . . . . . . . . . . . . . 33 
3 Meshless methods for high-speed impact $\quad 35$

3.1 SPH method . . . . . . . . . . . . . . . . . . 37

3.1 .1 SPH interpolation . . . . . . . . . . . . . . . . . . 37

3.1 .2 SPH instabilities and artificial viscosity $\ldots \ldots$. . . . . . . 37

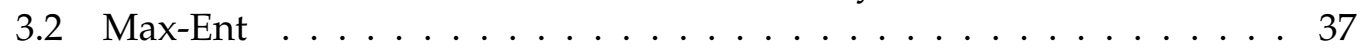

3.2 .1 Weak Kronecker-delta property . . . . . . . . . . . . . . . . . . 38

3.2 .2 Log-distance . . . . . . . . . . . . . . . . . . . . . . . . . . . 39

3.2 .3 Remeshing . . . . . . . . . . . . . . . . . . . 40

3.3 Meshless Analysis of High-Speed Impact/Contact Problem. . . . . . . 41

3.4 Explicit Contact $\ldots \ldots \ldots \ldots \ldots \ldots$. . . . . . . . . . . . . . . . . . . . . . . . .

3.4 .1 Contact detection . . . . . . . . . . . . . . . . . . . . . 45

3.4 .2 Correction geometry . . . . . . . . . . . . . . . . 46

3.4 .3 Correction forces . . . . . . . . . . . . . . . . . . . . . 47

3.4 .4 Velocity solution algorithm . . . . . . . . . . . . . . . . . . . . . . . . . . . . . . . . . . .

3.4 .5 Inelastic collisions $\ldots \ldots \ldots \ldots$. . . . . . . . . . . . 50

3.4 .6 Friction models . . . . . . . . . . . . . . . . . . . . 51

3.4 .7 Relation of the proposed algorithm with existing methods . . . 54

3.4 .8 Contact benchmarks . . . . . . . . . . . . . . . 55

Double elastic bar impact . . . . . . . . . . . . . . . 55

High velocity impact of a sphere onto a deformable plate . . . 56

Sliding block on an inclined plane $\ldots \ldots \ldots . \ldots 57$

Multi-body impact . . . . . . . . . . . . . . . . . 58

Taylor's bar impact . . . . . . . . . . . . . . . . . . . . . 59

3.4 .9 Oblique elastoplastic impact . . . . . . . . . . . . . . 61

3.5 Concluding remarks $\ldots \ldots \ldots \ldots$. . . . . . . . . . . . . 62

\begin{tabular}{|lr|r}
\hline & Simulations & 67
\end{tabular}

4.1 Ice impact onto leading edge $\ldots \ldots \ldots$. . . . . . . . . . . 67

$4.1 .1 \quad$ Projectile mass $\ldots \ldots \ldots \ldots \ldots$. . . . . . . . . . . . . . . . . . . . . . . . . . . .

4.1 .2 Projectile speed . . . . . . . . . . . . . . . . . 74

$4.1 .3 \quad$ Projectile strength . . . . . . . . . . . . . . . . . . . . . . . 75

4.1 .4 Projectile impact position . . . . . . . . . . . . . . . 77

4.2 Concluding remarks $\ldots \ldots \ldots \ldots$. . . . . . . . . . . 78

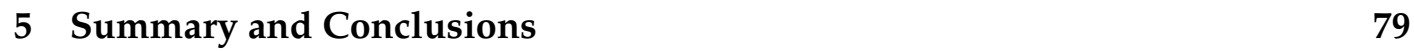

\begin{tabular}{|lr|}
\hline A Appendices & 83
\end{tabular}

A.1 Derivation of consistent tangent tensors . . . . . . . . . . . . . . . . . . 83

A.1.1 Tresca . . . . . . . . . . . . . . . . . . . 84

A.1.2 Drucker-Prager . . . . . . . . . . . . . . . . 85

\begin{tabular}{lr}
\hline Bibliography & 87
\end{tabular} 


\section{List of Figures}

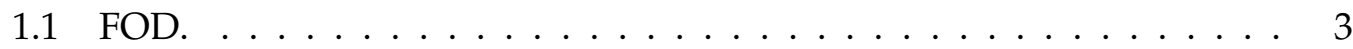

1.2 Hailstone impact on aircraft. . . . . . . . . . . . . . . . . 4

1.3 Largest hailstone found in Vivian, South Dakota, $20 \mathrm{~cm}$ in diameter. . . 4

1.4 Diverted flight to El Paso after severe damage in hailstorm, 3rd June

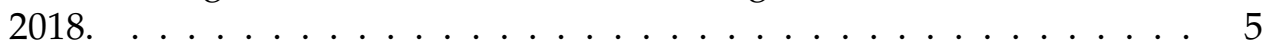

1.5 Bird-strike on aircraft. $\ldots \ldots \ldots \ldots \ldots \ldots$

1.6 Deformation of hailstone during impact. . . . . . . . . . . . . . . 7

1.8 Severe distortion in Lagrangian mesh. . . . . . . . . . . . . . . . . . 8

1.7 Hailstone impact with erosion. $\ldots \ldots \ldots$. . . . . . . . . . . . 8

1.9 SPH impact sphere with failure criteria. . . . . . . . . . . . . . . 10

2.1 Tresca yield surface (left) and Drucker-Prager's (right) in the space of principal stresses for $q=0$. The axis of hydrostatic stresses is shown

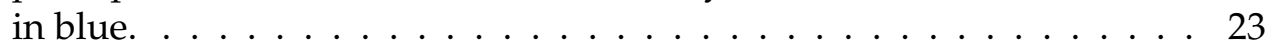

2.2 Strip footing geometry, boundary conditions, and loading. . . . . . . . 25

2.3 Forces on footing for different exponent values with Drucker-Prager

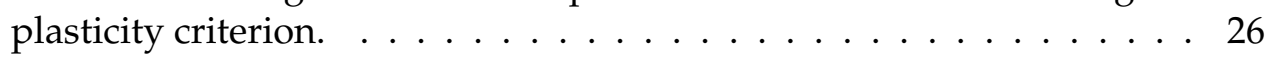

2.4 Strip footing simulation. Pressure to cohesion ratio as a function of time. Pressure in the strip footing and theoretical solution for Tresca

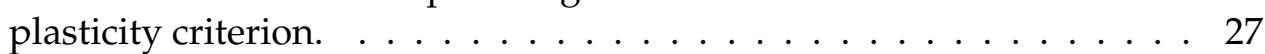

2.5 Dissipation of the strip footing problem performed with tresca plasticity model and mesh with $80^{2}$ elements. This dissipation is the volume integral over the body of the dissipated energy at each integration point. 27

2.6 Forces for different values of relaxation time in cylinder traction test..$~ 28$

2.7 Forces for different strain rates in cylinder tensile test for $\tau=5 \cdot 10^{-5} \mathrm{~ms}$. 28

2.8 Effective plastic strain in perforated plate. From left to right, top to bottom, solutions at time $t=78,145,278$ and $331 \mathrm{~ms}$ for test with $n=5.29$

2.9 Forces for different values of exponent $n$ in the material of the perfo-

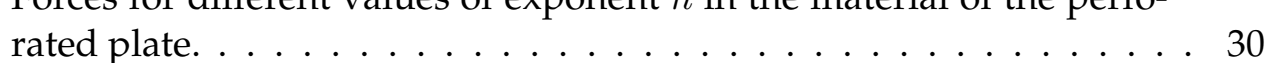

2.10 Forces for Taylor's bar impact . . . . . . . . . . . . . . . . . . . 31

2.11 Effective plastic strain in Taylor's bar impact. From left to right, top to bottom, solutions at time $t=0,8,12,16 \mu \mathrm{s} . \ldots \ldots \ldots 32$

2.12 Neohookean sphere impacting onto a rigid plate, form left to right using LS-DYNA and MUESLI constitutive material respectively. . . . . 33

3.1 DEM quadratic shape functions in interval $[0,1]$ for 11 nodes. . . . . . . 36

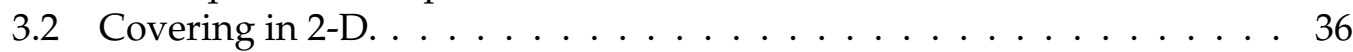

3.3 Non-convex domain . . . . . . . . . . . . . . . . . . . . . . . 39

3.4 Ball for node search. . . . . . . . . . . . . . . . . . . . . 41

3.5 The two types of bodies considered are meshed (left) and meshfree (right). Bodies with a mesh have a well-defined skin of elements intersecting the boundary. . . . . . . . . . . . . . . 46

3.6 Two types of skin penetration in plane problems. . . . . . . . . . . . . 47 
$3.7 \quad$ Flowchart for the contact algorithm. . . . . . . . . . . . . . . 56

3.8 Double Taylor bar impact, Von Mises stress (Pa). From left to right, solutions at time $t=1,5,9,12,16,21 \mu \mathrm{s} . \ldots \ldots \ldots \ldots 7$

3.9 Von Mises stress (Pa) of the sphere impact onto deformable plate. From left to right, top to bottom, solutions at time $t=5,32,80,132,200,302$

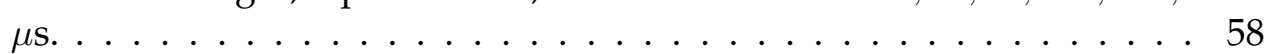

3.10 Normal force exerted on the plate. . . . . . . . . . . . . . . . . 59

3.11 Meshfree cube on inclined plane. . . . . . . . . . . . . . . . . . . . . . 59

3.12 Kinetic energy of the sliding cube on an inclined plane. . . . . . . . . . 60

3.13 Arrangement of finite element / meshfree cubes. . . . . . . . . . . . . . 60

3.14 Linear momenta evolution for multi-body impact. . . . . . . . . . . . . 61

3.15 Total energy evolution for multi-body impact. . . . . . . . . . . . . . 61

3.16 Von Mises stress (Pa) for multi-body impact. From left to right, top to bottom, solutions at time $t=19,49,79,128,295,376 \mu \mathrm{s}$. . . . . . . . 63

3.17 Taylor's anvil test. From left to right, top to bottom, deformed configurations at time $t \sim 11.2 k \quad \mu \mathrm{s}$, with $k=0,1, \ldots, 5$, showing the

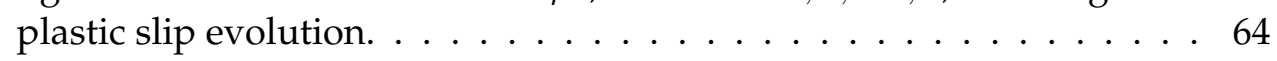

3.18 Elastoplastic of a sphere on plate. Initial configuration, showing the finite element mesh and meshless body. . . . . . . . . . . 65

3.19 Impact of elastoplastic sphere. From left to right, top to bottom, deformed configurations at time $t \sim 4 \cdot k \quad \mu \mathrm{s}$, with $k=0,1, \ldots, 5$, showing the plastic slip evolution and cut by the plane of symmetry. $\ldots .65$

3.20 Linear momentum components of the sphere during the impact of

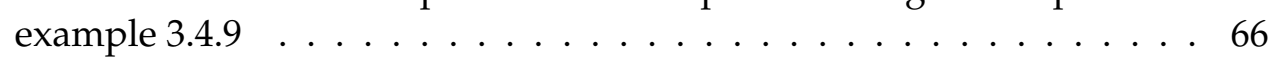

3.21 Total energy of the sphere during the impact of example 3.4 .9 . . . . . 66

4.1 Leading edge. . . . . . . . . . . . . . . . . . . . . . 6 . . . . . . . . 67

$4.2 \quad$ Discretised leading edge. . . . . . . . . . . . . . . . . . . . . 68

4.3 Discretised leading edge. . . . . . . . . . . . . . . . . . . 68

$4.4 \quad$ von Mises stress evolution in Reference 1 from left to right top to bottom at 36, 50,75 and $200 \mu \mathrm{s},(\mathrm{Pa}) . \ldots \ldots \ldots . \ldots . \ldots 70$

4.5 Plastic slip evolution in Reference 1 from left to right top to bottom at $40,100,160$ and $200 \mu \mathrm{s} . \ldots \ldots \ldots \ldots \ldots \ldots \ldots$

$\begin{array}{ll}4.6 & \text { Force modulus exerted onto both constraints of the leading edge in } \\ & \text { Reference 1. } \ldots \ldots \ldots \ldots \ldots \ldots \ldots \ldots \ldots \ldots \ldots \ldots \ldots\end{array}$

$4.7 \quad$ Ice mesh distortion evolution from left to right, top to bottom at 46, 90, 150 and $200 \mu \mathrm{s}$ in Reference 1. . . . . . . . . . . . . . . . 72

$4.8 \quad$ Force modulus on one of the constraints Reference 1, Reference 2 and

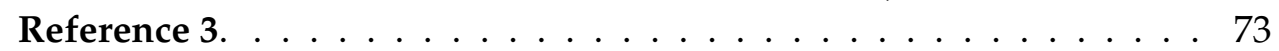

4.9 Plastic strain comparison, from left to right Reference 1, Reference 2 and Reference 3. . . . . . . . . . . . . . . . . 73

4.10 Plastic damage comparison. . . . . . . . . . . . . . . . . . 74

4.11 Force modulus on one of the constraints Reference 1, Reference 4 and Reference 5. . . . . . . . . . . . . . . . . 74

4.12 Plastic strain comparison, from left to right Reference 1, Reference 4 and Reference $5 . \ldots \ldots \ldots \ldots \ldots 75$

4.13 Plastic damage comparison. . . . . . . . . . . . . . . . . 75

\begin{tabular}{|l}
4.14 Force modulus on one of the constraints Reference 1, Reference 6 and \\
Reference $7 . \ldots \ldots \ldots \ldots \ldots$
\end{tabular}

4.15 Plastic strain comparison, from left to right Reference 1, Reference 6
and Reference $7 . \ldots \ldots \ldots \ldots \ldots \ldots \ldots \ldots \ldots \ldots \ldots \ldots \ldots \ldots \ldots$ 
4.16 Plastic damage comparison. . . . . . . . . . . . . . . . . . . . . 77

4.17 Force modulus on one of the constraints Reference 1, Reference 8 and

Reference 9. . . . . . . . . . . . . . . . 77

4.18 Plastic strain comparison, from left to right Reference 1, Reference 8

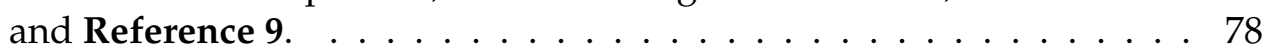

4.19 Plastic damage comparison. . . . . . . . . . . . . . . . 78

A.1 On the left, cross section of the yield surface of Tresca's model in principal stress space, as viewed from the hydrostatic axis, and identification of one of the six sextants that partition the space. On the right, on a generic sextant, the three possible projections of the stress onto the yield surface (left vertex, center plane, and right vertex). For simplicity, no isotropic hardening is considered. . . . . . . . . . . . . . . 84 



\section{List of Tables}

2.1 Material parameters for viscoplastic Drucker-Prager model for strip

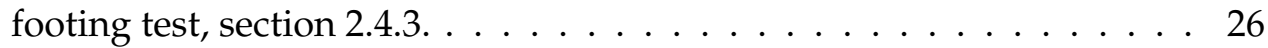

2.2 Material parameters for viscoplastic Tresca model in strip footing test,

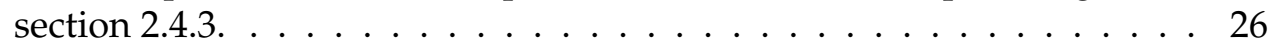

2.3 Material parameters for viscoplastic Tresca model in traction test, sec-

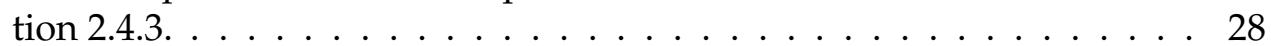

2.4 Material parameters for viscoplastic Tresca model in perforated plate, Sec.|2.4.3| . . . . . . . . . . . . . . . . . . . . . . 29

2.5 Material parameters for viscoplastic Tresca model in Taylor bar, sec-

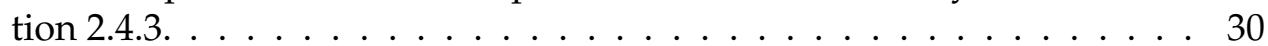

3.1 Johnson-Cook constants for copper. . . . . . . . . . . . . . . . 60

3.2 Johnson-Cook constants for steels. . . . . . . . . . . . . . . . . . 62

4.1 Tresca viscoplastic model for leading edge. . . . . . . . . . . . . . . . . 69

4.2 Drucker-Prager viscoplastic model for ice sphere. . . . . . . . . . . . . 69 



\title{
List of Abbreviations
}

\author{
ALE Arbitrary Lagrangian Eularian \\ DEM Diffuse Element Method \\ EOS Equation Of State \\ FEM Finite Element Method \\ FOD Foreign Object Damage \\ LME Local Max Ent \\ MEF Método de los Elementos Finitos \\ NEM Natural Element Method \\ PUM Partition of Unity Method \\ RKPM Reproducing Kernel Particle Method \\ SPH Smoothed Particle Hydrodynamics
}



Dedicated to my parents and sister who are the most precious thing in my life. 



\section{Chapter 1}

\section{Introduction}

\subsection{Motivation}

High velocity impact phenomena can be found in many engineering situations. For instance, in aeronautics, aircrafts can encounter foreign objects such as birds and hailstone during their operation. Usually, these events are more likely to happen during take-off and landing, where velocities are at maximum $80 \mathrm{~m} / \mathrm{s}$. Also within the domain of aeronautics, rockets may encounter this event but at higher velocities in launch and re-entry stages where velocities may reach some thousands of $\mathrm{m} / \mathrm{s}$ as well as satellites in orbit might be impacted by space debris at velocities around $500 \mathrm{~m} / \mathrm{s}$. The same event can be found in ballistics where velocities can attain in modern projectiles $1,200 \mathrm{~m} / \mathrm{s}$. However one may find that the definition of high speed velocity is vague and the range of what is defined as high velocity seems to be very wide. When high velocity impact is used, it means that the impact velocity is either approximately of the same order or greater than the wave speed of the impacted material. This quantity is defined as:

$$
c=\sqrt{\frac{E}{\rho}},
$$

where $E$ is the Young's modulus and $\rho$ is the density of the impacted material, respectively. The consequences of these impacts can be devastating, causing severe damage in the impacted elements. For this reason, in the last decades there has been a major and increasing concern, fostered to a certain extent by:

- A widespread worldwide flight network which according to the International Air Transport Association has around 37.5 million flights per year, meaning that the odds of having impacts of any nature are high, e.g. the emergency landing in Hudson river caused by a flock of Canada geese, 2009.

- Ballistics in modern warfare.

- The restart of the Space Race with new commercial space companies as SpaceX and an increasingly interest of planetary exploration.

- The increasing amount of space junk in orbit around Earth, making this region collision-prone for new space launches.

This concern is based on the economical costs involved in the events mentioned above. Without forgetting the possible human cost as a consequence of impact, leading for instance to a sudden stop of aircraft engines during its operation, forcing risky landings. The structural damage known as FOD is any damage attributed to a foreign object (i.e. any object that is not part of the vehicle) that can be expressed 
in physical or economic terms and may or may not degrade the product's required safety or performance characteristics [1]. Aircraft turbine engines routinely experience the ingestion of debris resulting in FOD [2]. Failures associated with this kind of damage have been estimated to cost the aerospace industry $\$ 4$ billion per year. Often, its consequences do not lead to sudden catastrophic failure, yet such damage can dramatically reduce the life of components subjected to cyclic fatigue stresses. Turbine blades, for example, are susceptible to debris strikes and also experience significant fatigue loading (see Fig. 1.1). Examples of FOD include:

- Aircraft parts, rocks, broken pavement, ramp equipment.

- Parts from ground vehicles

- Garbage, maintenance tools, etc. mistakenly or purposely deposited on tarmac and/or runway surfaces.

- Hail.

- Ice on the wings, propellers, or engine intakes

- Dust or ash clogging the air intakes (as in sandstorms in desert operating conditions or ash clouds in volcanic eruptions).

- Tools, bolts, metal shavings, lockwire, etc. mistakenly left behind inside aircraft during the manufacturing process or maintenance.

Given the wide range of debris, impact locations and velocities, a complete experimental test or even a whole numerical analysis of the different impacting bodies would be prohibitively expensive and cannot be accomplished in a timely manner. Therefore, it was deemed to focus this work on the two most common foreign objects i.e. ice and bird strike. Thus, the understanding of the possible loads to which structural parts might be subjected during high-velocity impact becomes essential in order to assess the possible structural damage and be able to mitigate it. This implies an economical effort to set expensive experimental tests, for instance the use of gas reservoirs and long gas canons to accelerate the projectiles. Moreover due to the limitations of a certain experimental set-up the number of possible test and its variety is restricted. Therefore the study of this phenomena from a numerical perspective by means of computational mechanics opens up numberless possibilites and conditions to be tested with an insignificant extra cost. In this regard, the work of this dissertation starts within the context of the project SIMUFOING, entitled: "Development and validation of simulation methods for ice and bird ingestion in airplane engines". This project between IMDEA Materials and Industria de Turbopropulsores (ITP) is aimed at the development of new simulation techniques that can be used to predict damage on aircraft engines due to the ingestion of hailstones and birds. The numerical methods will be validated with experimental data provided by ITP, and should be accurate enough to estimate structural failure of engine parts at a wide range of ingestion velocities. The Computational Solid Mechanics group at IMDEA Materials will be responsible for the dynamic simulations and the material model implementations to understand from a numerical approach the impact of foreign objects in the first stage of a jet turbine. 


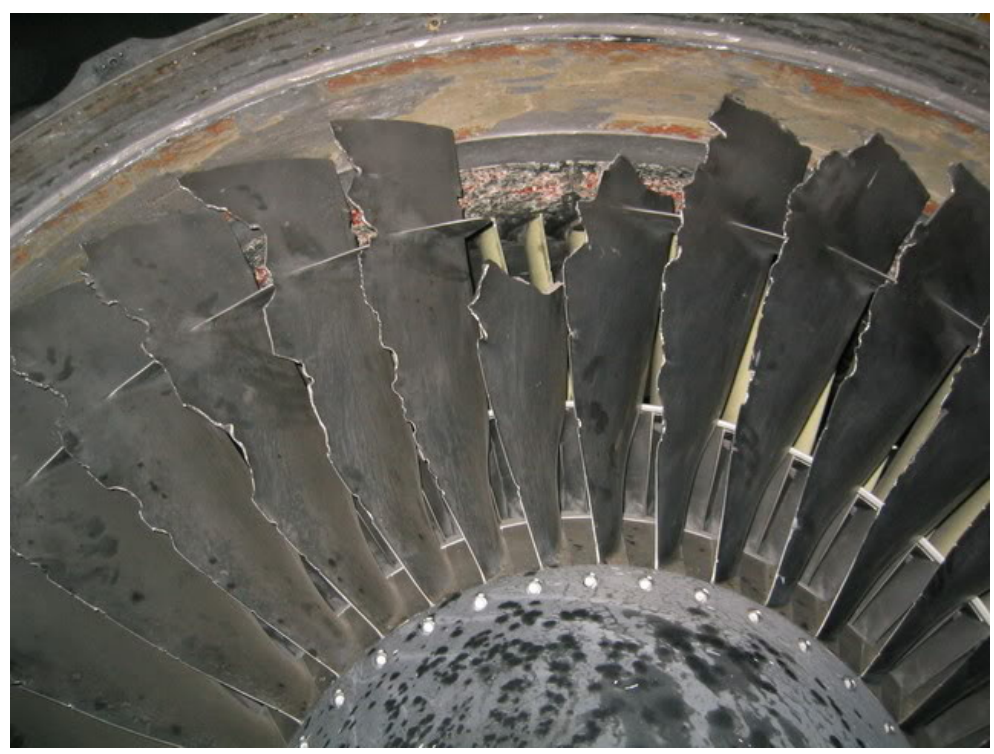

FIGURE 1.1: FOD.

\subsubsection{Ice impact}

Hailstone impact is still nowadays a threat for the integrity of aircraft structures such as the airframe, engine nacelles, fan blades and so forth. Therefore, it becomes important to know how high-velocity hail impacts behave and can damage the structure during operation. Hail impacts threaten the structural integrity of the aircraft, since impacts can cause serious damage to structures, such as abrasions, dents and, in some cases, perforations, see Fig. 1.2. This concern dates back to the beginnings of aerospace engineering, since the early 1950s [3]. Some cases have been reported with significant damage, e.g. [4], a storm that dropped hailstones of a size of 100 $\mathrm{mm}$ diameter some larger examples were found in Vivian, South Dakota during a thunderstorm on 23th July 2010, see Fig. 1.3. As a very recent accident we mention the flight heading to Phoenix from San Antonio, Texas, that was forced to land in El Paso due to damage from a hail storm the 3rd June 2018, see Fig. 1.4. This has motivated to conduct a plethora of experimental tests to understand the failure and survival of metal and composite structures under hail impact in order to assist redesign of structural parts improving their performance in these situations.

There is a large literature that describes the structure and mechanical behaviour of ice, as a main reference, we can mention Schulson et al. [5]-[7]. Ice should be regarded as a class of materials rather than a single material with well defined properties. There are thirteen different crystal structures and two amorphous states. The ordinary ice is called ice Ih and has a hexagonal crystal structure that forms at ambient pressures. Like many brittle materials, the strength in compression is stronger than in tension, although unlike most brittle materials, it exhibits its brittle behaviour up to the melting point. It has been reported compressive strengths of $14.8 \pm 2.3 \mathrm{MPa}$ in its single crystal form at $-10^{\circ} \mathrm{C}$ and tensile strengths with a wide scatter from 0.7 to 3.1 $\mathrm{MPa}$ over a temperature range of $-10^{\circ} \mathrm{C}$ to $-20^{\circ} \mathrm{C}[5],[8]$. The strength of ice is dependent on the crystal structure and also on pressure, with an increase in strength as confinement increases. Also, a remarkable property of ice, is being a strain rate dependent material in compression [9], showing a ductile behaviour for low strain rates and a brittle behaviour for higher strain rates [10]. This ductile to brittle transition occurs at a strain rate on the order of $10^{-3} \mathrm{~s}^{-1}$ at temperatures on the order 
of $-10^{\circ} \mathrm{C}$. Within the range of impact velocities, several studies have been made providing insight about ice behaviour in these conditions [11]-[14]. Since strain rates in impact are significantly higher than the ductile to brittle transition strain rate, ice failure is brittle [15], with an increase in compressive strength as strain rate increases. Regarding tensile behaviour, experimental results [8], show brittle failure and a negligible influence of strain rate in the strength of ice.

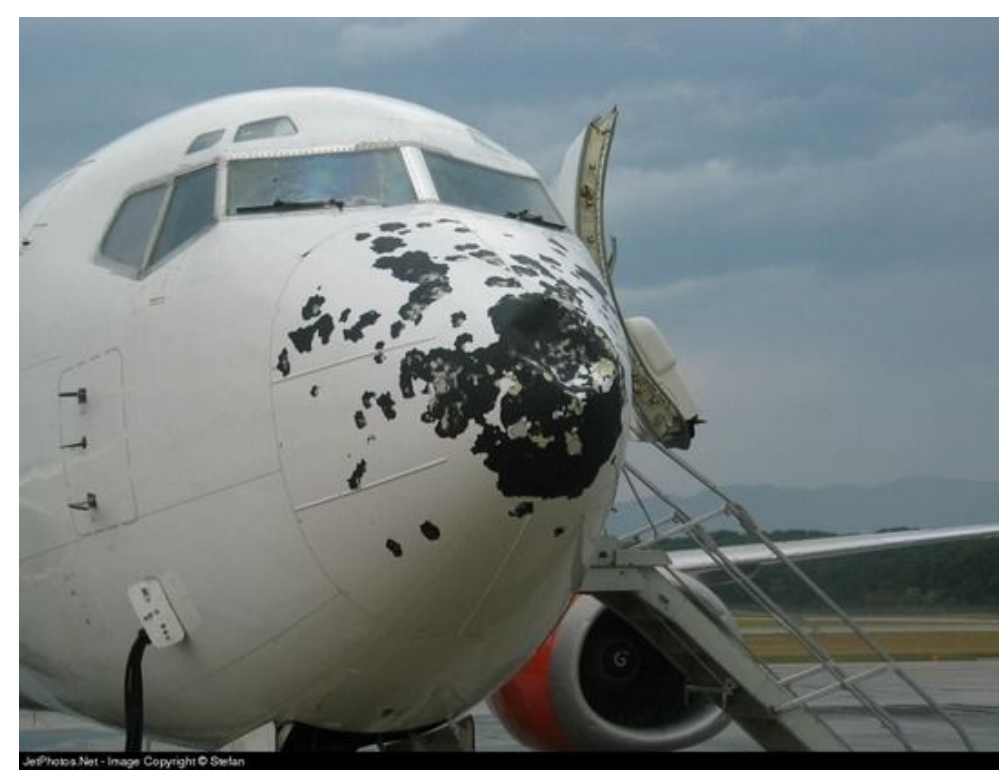

FIGURE 1.2: Hailstone impact on aircraft.

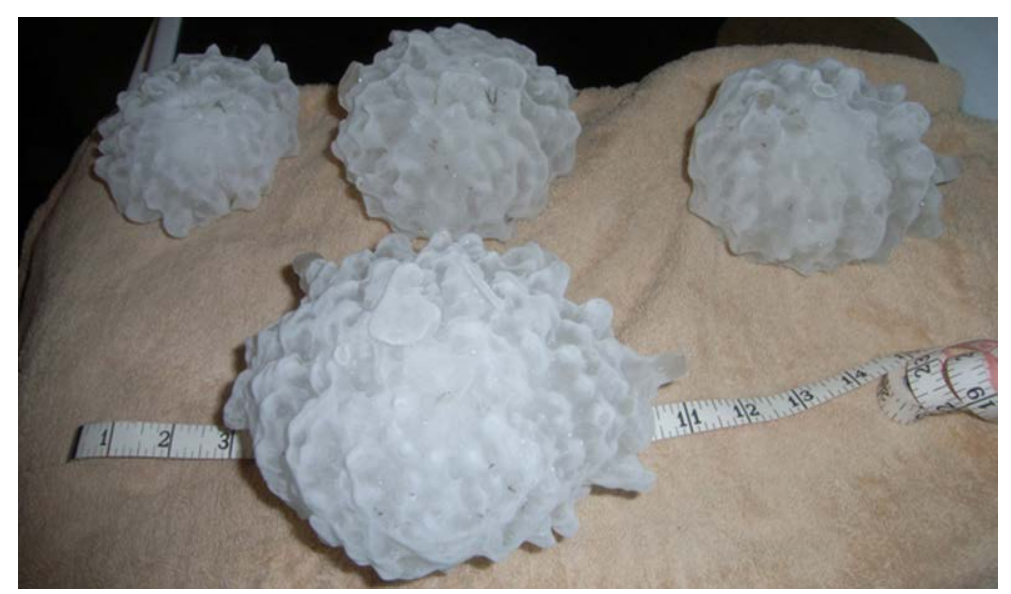

FiguRE 1.3: Largest hailstone found in Vivian, South Dakota, $20 \mathrm{~cm}$ in diameter.

\subsubsection{Bird Impact}

Bird strike in aeronautics is any contact between an aircraft and any flying living creature, see Fig. 1.5. The first bird strike dates back to 1905, today the Federal Aviation Administration receives more than 10,000 reports of bird strike each year, however they estimate that this is only a $50 \%$ of all strikes that occur. This has an estimated cost of $\$ 600$ millon to civil aviation each year. Furthermore, more than 250 people have lost their life as a consequence of bird strike since 1988 [16]. The results 


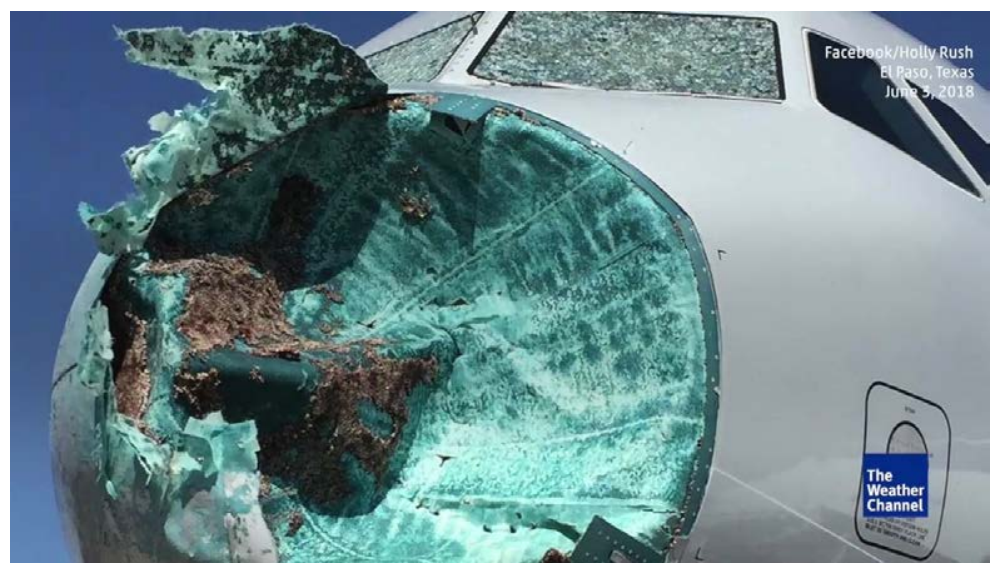

FIGURE 1.4: Diverted flight to El Paso after severe damage in hailstorm, 3rd June 2018.

of such impact can be catastrophic leading to indentations or even small holes in the frame. All forward facing components of the aircraft can be subjected to bird strike, for instance, the engine fan blades, the windshield, the leading edges of the wings, etc [17]. Therefore bird impact is a major threat to safety in aeronautics, in fact, more than $90 \%$ of FOD is due to bird strike [18]. This has promoted a wide study of the phenomena with experimental tests trying to reproduce the event, theoretical works an numerical investigations. Initially, bird projectiles in experimental tests were represented with dead chickens, however it was demonstrated that equivalent results could be obtained using gelatine [19], [20], which leads to less dispersion and more homogeneous results, avoiding at the same time the difficulties involved in dead animal handling. To conclude, we could highlight some existing reviews [17] and monographs [21] that thoroughly analyse the issue of bird strike.

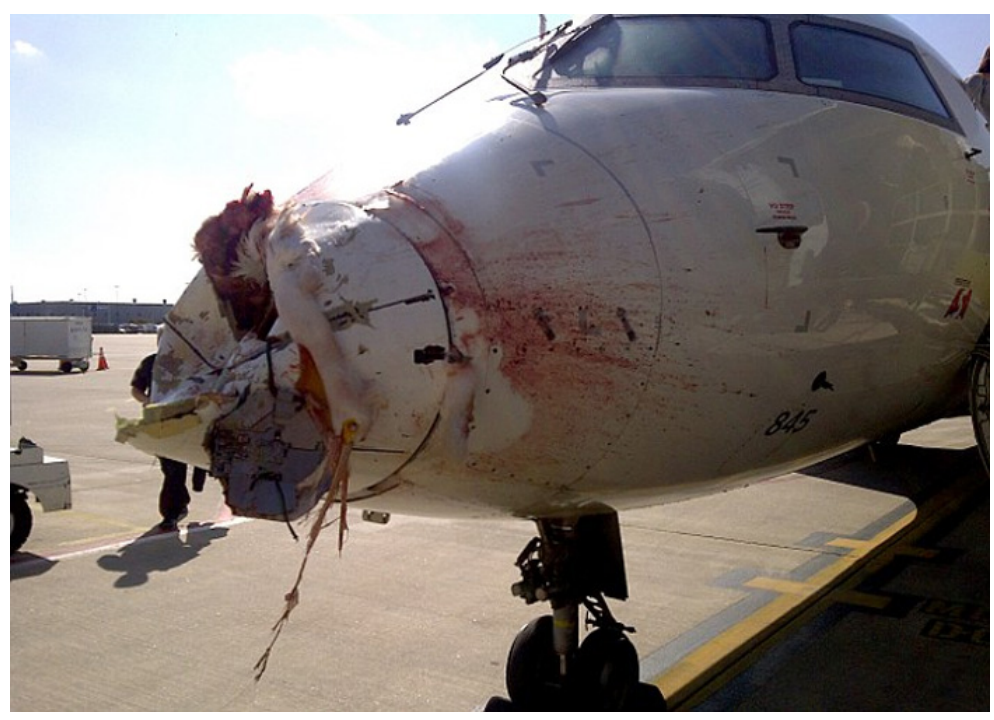

FIGURE 1.5: Bird-strike on aircraft.

\subsection{State of the art}

As mentioned in Section 1.1, this work takes a numerical perspective on the study the issue of ice and bird strike, involving the use of different parts from the body of 
knowledge that is computational mechanics. Let us briefly sum up the tools needed to simulate this event:

- Contact: There exists a physical interaction between the impacting body and the structural parts.

- Failure criteria: The common feature between bird and ice as impacting objects is that they are ranged within a wider family coined as low-strength projectiles. When a projectile of this type impacts, the particles on the impacting surface are instantaneously brought to relative rest to the impacted target, this generates a shock wave with very high pressure. This pressure gradient causes the particles to accelerate radially (perpendicular to impact direction) causing shear stress, if at any time the stress state is such that the material strength is exceeded, as expected with low strength materials, the material will flow thus after failure they might be considered to behave as fluids [19]. Thus, a certain failure criteria has to be included that accounts for an stress state threshold from where the stress tensor is modified to behave as a fluid, i.e. no tensile stress state. Their implementation for explicit schemes is relatively easy. There is a wide variety of other options for this threshold, e.g. pressure, strain energy and others [22]. An alternative is the so-called eigenerosion method [23] which is an erosion criteria based on the restriction of eigendeformations in a binary sense.

- Explicit integration schemes: Since elapsed times during these events are very short and dynamic response is dominant, explicit integration schemes are the standard choice.

- Constitutive model: as mentioned before, ice is a rate dependent material, and since the range of impact velocities might vary a general class of materials with a time dependent response seems to be the best choice, this hints to us that elasto-viscoplastic models will might be good constitutives laws for simulate the response of ice and bird.

- Spatial discretisation methods: Due to the hydrodynamic behaviour just after impact, large distortions in the impacting bodies are expected. Ideally, the best choice for spatial discretisations are those that do not suffer from bad accuracy when the initial configuration is severely distorted.

The literature for modelling high velocity impacts is, to a certain extent, small. This is not surprising considering that only recently the computational power for this type of simulation is available and the market for this field of research is a narrow segment in the aerospace industry. Most of the existing literature modelling this phenomena uses LS-DYNA [24]. Thus, given the available methods in LS-DYNA, three approaches for modelling ice and bird impact can be found in literature. These are FEM (Lagrangian approach), ALE, and SPH [17], [25].

\section{Lagrangian finite element approach}

The Lagrangian FE approach is typical for continuum mechanics and strongly recommendable for the analysis of impact events. This approach is extremely efficient when considering non-linear problems, though it has its weak point in the excessive mesh distortions, which are rather typical events featuring soft-bodies or fluid-like materials as in the case considered. Following a Lagrangian approach, if the material undergoes large distortions, since the mesh follows the material movement, it 
will be distorted too, see Fig. 1.6. Since the accuracy of FEM is related to the element aspect ratio, large distortions of the mesh might cause an unacceptable loss in accuracy. Moreover, in order to fulfil the CFL condition if the element length decreases, the timestep will decrease, increasing the required CPU time. This drawback can be overcome by an erosion criterion which means that when an element fails fulfilling a given failure criteria, the element is deleted automatically. However, it produces damage far from the evidences collected after real impacts, see Fig. 1.7. Moreover element deletion produces a mass reduction which might spoil the truthfulness of the results. Using this approach in ice simulation we can mention the work of Kim et al. [26] to simulate high-velocity ice impact. The work of Kim et al. was also applied for the study of damage in composite joints [27]. Subsequently a more realistic model was developed by Carney et al. [28], [29]. They use an Eulerian approach instead of a Lagrangian mesh which avoids mesh distortion after impact. The interactions between target and ice are done through a penalty contact method, with a strain rate dependent ice material. The authors use a failure criteria based on a pressure cut-off, after which an EOS is used to determine the behavior of the crushed ice. Other works use a damage model to reproduce brittle cracking during impact like in [30]. For bird impact Airoldi et al. [31] used a Lagrangian approach with a trial-and-error procedure for the elimination of the excessively distorted elements.
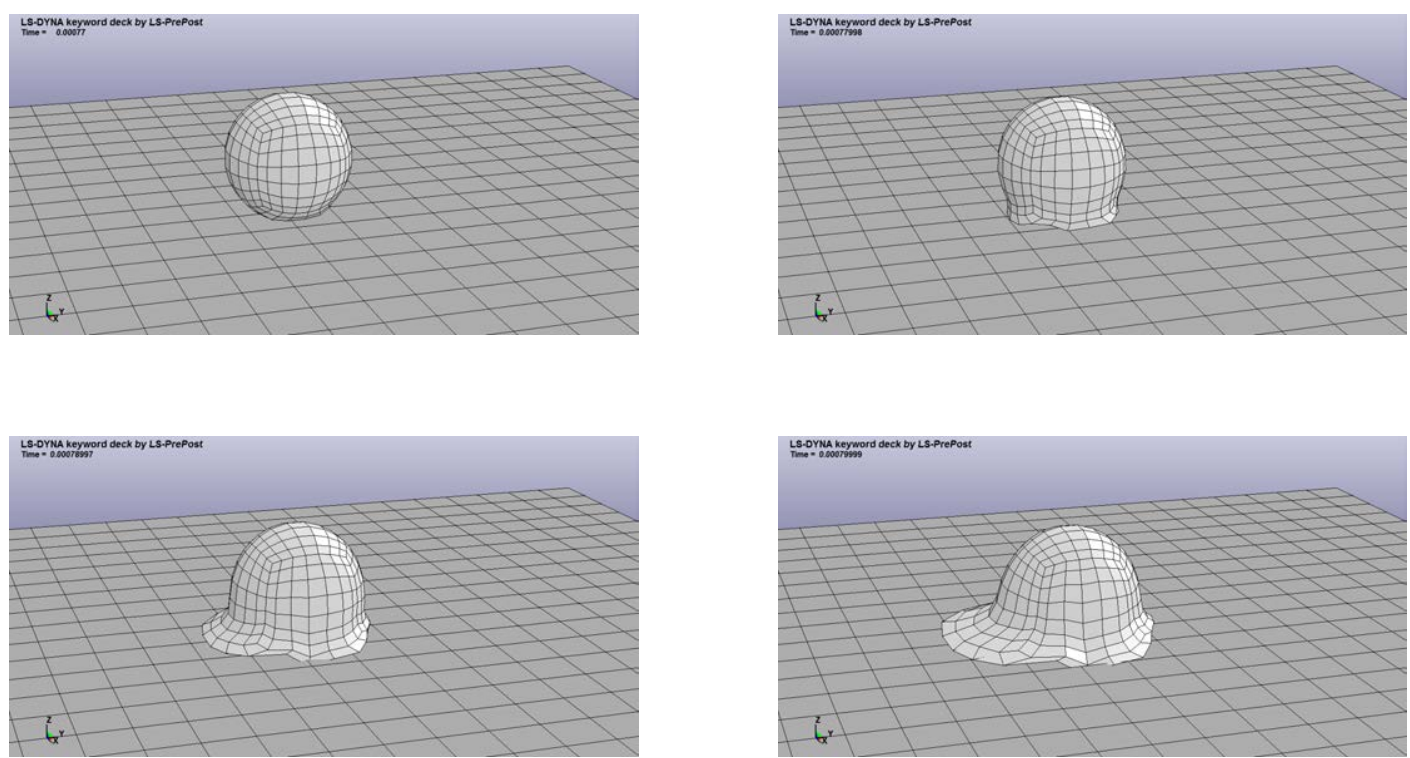

FIGURE 1.6: Deformation of hailstone during impact. 


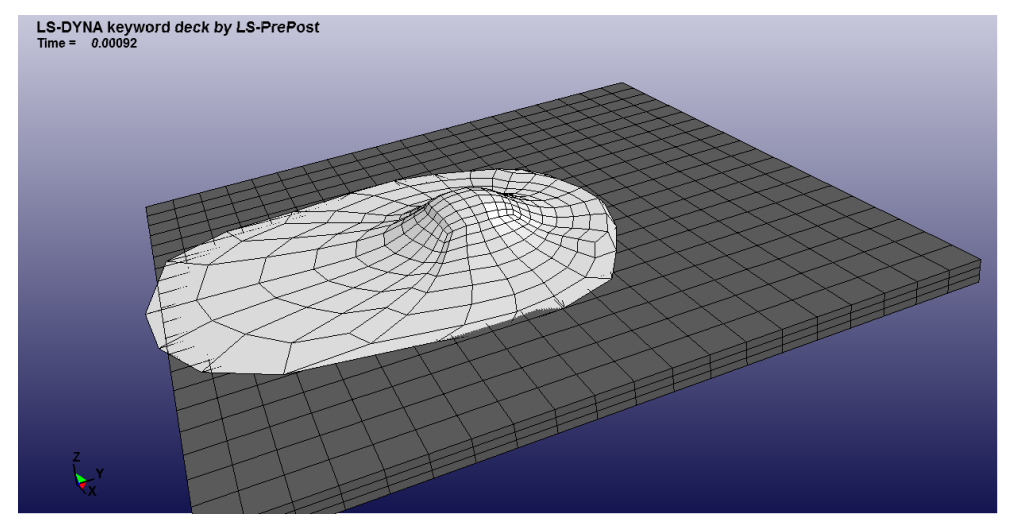

FIGURE 1.8: Severe distortion in Lagrangian mesh.
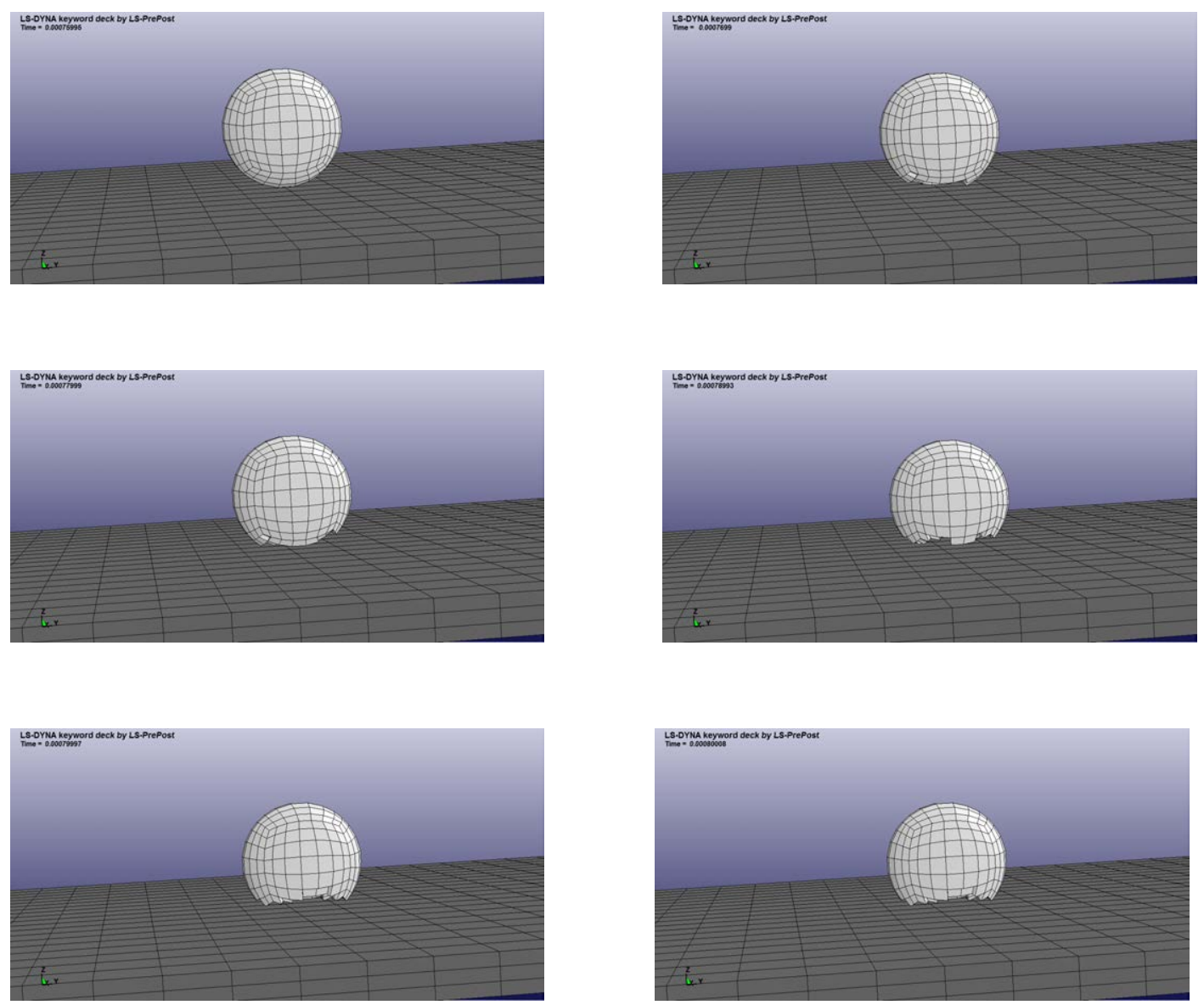

FIGURE 1.7: Hailstone impact with erosion.

\section{Arbitrary Lagrangian-Eulerian approach}

The ALE approach is meant to combine the advantages of both Lagrangian and Eulerian approximation. Following an Eulerian approach, the material flows through a mesh fixed in the space. The ALE method differs in that the Eulerian mesh can move arbitrarily and independently on the motion of the material. Consequently, it has an advantage over the pure Eulerian approach when the motion of the material covers a wide region of the space. Some of the works that have employed this method for 
ice impact are [25], [32], in the case of bird impact simulation we can cite [20] and the work of Hanssen et al. [33] that employs a subroutine defined in LS-DYNA.

\section{Smoothed particle hydrodynamics approach}

The SPH method was initially developed, see [34], [35], to study astrophysical and cosmological phenomena. Subsequently, it was extended to typical problems of computational fluid dynamics (CFD) and finally to continuum mechanics. The main difference between SPH and FE methods is related to the discretisation of the continuum. SPH is a meshless method, thus the mesh is replaced with a set of particles endowed with a mass and no direct connectivity exists among them, see Fig. 1.9. Some of the works that have been done to reproduce the impact of brittle materials are [36] that uses the PINBALL contact algorithm [37]. We can cite other works like [38] where ice is modeled with a constitutive model included in LS-DYNA and [32] that uses a Drucker-Prager plasticity criteria and a compressive yield stress that varies with the strain rate which makes the model pressure dependent, as ice behaviour. For bird impact we can cite [20] and also [39], where the authors use a Hugoniot equation of state (EOS) for the bird, having a fluid-like behaviour.

\section{Concluding remarks on available spatial discretisations}

In particular, the FE model proves to be suitable to reproduce only the early stages of the impact when the distortion of the mesh is not such to cause inaccuracy, drastic drops in time-step size or premature analysis termination. An erosion criterion could be a solution to avoid excessive mesh distortion but still the mass reduction is a drawback, therefore this approach can be discarded. In contrast, the time step when using the ALE model remains roughly constant throughout all the simulation. Unfortunately, it suffers from the typical problems when coupling solvers based on different approaches, namely advection introduced errors. The SPH model proves to be the most effective. In fact, this model grants a close numerical-experimental correlation and the required CPU-time is considerably smaller than the one required by the two other models. Furthermore, the SPH model effectively reproduces the hailstone behavior also when the hailstone cracks after impact. However SPH as meshless method is well known for its instabilities as well as it lacks from an underlying convergence theory. Thus, although meshless methods are the best choice for low-strength projectiles, other options must be investigated. In this context we can cite the work of [40], [41] that uses other meshfree methods known as Max-ent functions and were also applied to the simulation of high speed impact.

\subsection{Main lines of work}

This work has identified several lines of improvement in the state of the art for ice and bird impact. This led to several lines of work which are the development of more general elasto-viscoplastic constitutive models for non-smooth plasticity, prepare interfaces thar are amenable to link user subroutines with FEM solvers, set the tools for the implementation of a proper meshless method and finally the implementation of a better conditioned contact algorithm. With this roadmap we expect to achieve a robust methodology for high speed impact. Finally we thoroughly develop in the following paragraphs the lines of work. 

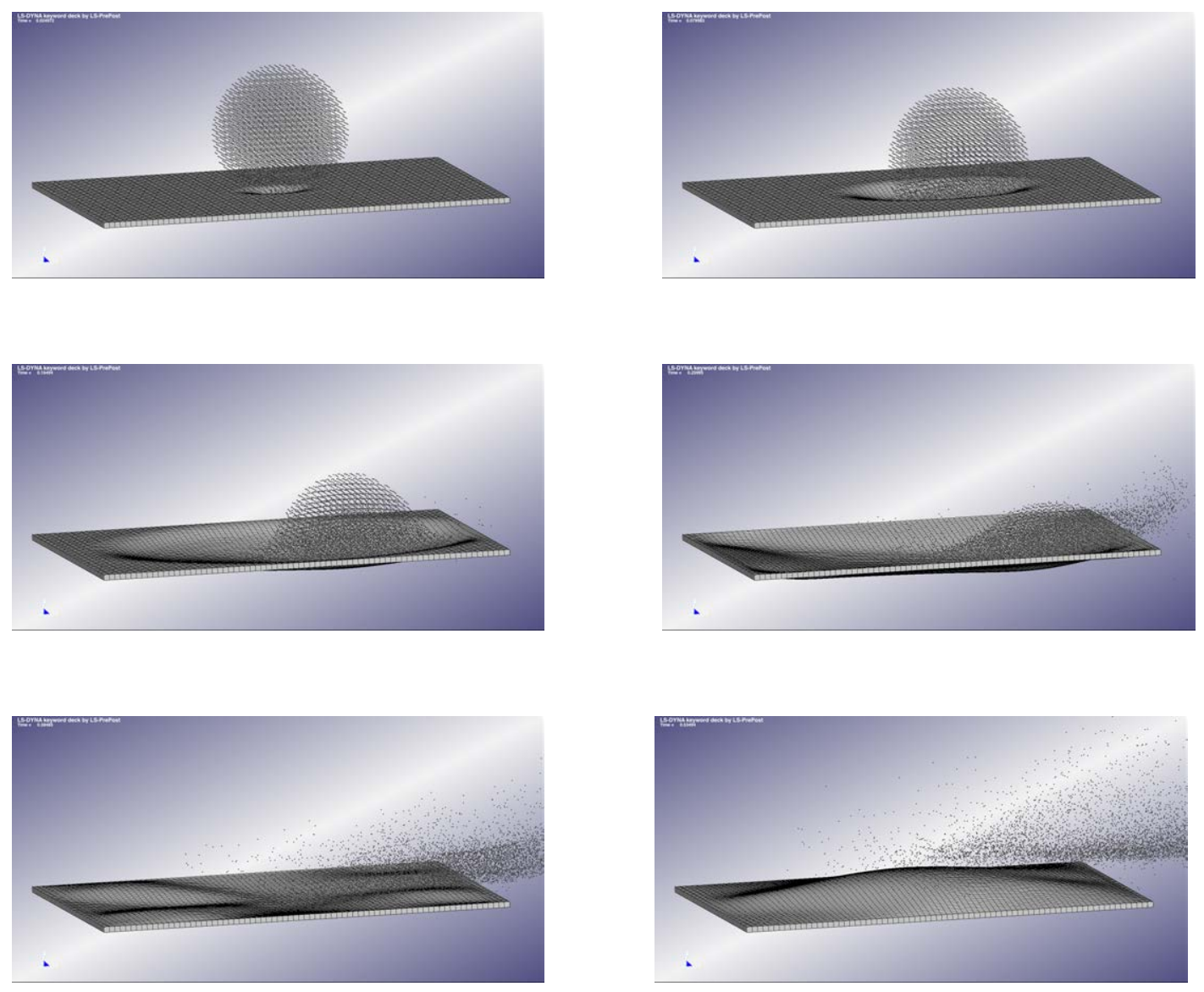

FIGURE 1.9: SPH impact sphere with failure criteria. 


\subsubsection{Constitutive models}

As mentioned in Section 1.2, the use of rate dependent materials that are based on a certain plasticity criteria seems to be a good choice for several reasons:

- Elasto-viscoplastic materials are general classes of materials that embody other smaller classes like elastic and plastic constitutive models, thus its use is amenable to reproduce a wider variety of behaviours.

- As seen in section 1.1.1 ice is a rate dependent material.

- Impact implies a wide range of velocities, it would be expected to have different results at different velocities, this is to a certain extent possible with rate dependent materials.

However, to the author's knowledge, the rate dependent materials seen in the literature for ice and bird impact are based on flow stress models which are yield functions that may vary with the strain rate, e.g. Johnson-Cook. This yields a pseudoviscoplastic behaviour, since in fact it still relies on a plastic model, the yield function is never greater than zero. This seems to the author a not consistent approach since it does not rely on an energy principle. However, there is another approach that uses a penalty formulation for the principle of maximum plastic dissipation, the socalled viscoplastic regularisation. With a proper choice of the penalty function, it can be proved mathematically that the second law of thermodynamics is preserved. As a consequence of this approach, the stress state can lie outside the yield function. We will address this issue in a very extensive and thorough way in Chapter 2, giving the mathematical setting of viscoplasticity and introducing further concepts, such us non-smooth viscoplasticity. Later we will address non-smooth viscoplasticity using two non-smooth plasticity criteria, i.e Tresca and Drucker-Prager. Finally we will provide benchmarks to show its performance.

\subsubsection{Interfaces for material subroutines}

Due to the widespread use of commercial codes and mainly the use of LS-DYNA to simulate ice and bird impact we developed a series of interfaces in $\mathrm{C}++$ to link the constitutive models. Later, we realised the necessity in the computational mechanics community of a material library that would able to link to open source finite element codes or commercial codes like Abaqus and LS-DYNA a wide variety of constitutive models. This, led to the development of MUESLI which embodies materials laws for elasticity and plasticity in small strain and finite strain theory, thermoelastic, viscoelastic materials and so on. Moreover MUESLI has other remarkable features, one of the major advantages is that due to the use of an object oriented programming structure the implementation of new materials is very easy. We should also mention there exists a series of buit tests to check the correctness of the subroutines implemented. We will address in a more extensive way the features of MUESLI in Section 2.6.

\subsubsection{Spatial discretisation methods}

Regarding the spatial discretisations we concluded that for high speed impact of low strength projectiles, a large distortion of the body is expected, thus particle or meshless methods are the proper choice. However, the use of SPH will be discarded due to its pathological properties. Other options relying on a convergence theory 
will be investigated in Chapter 3 to conclude that meshless methods with Kronecker delta property are the best choice, since these methods facilitate the imposition of essential boundary conditions and in the case of contact, which is a cornerstone in impact, it will let an straightforward manipulation of nodal kinematic quantities.

\subsubsection{Explicit contact}

For explicit contact Lagrangian multipliers cannot be directly employed and the formulation has to be adapted. Thus, the most extended contact method is the penalty method. Nevertheless, it suffers from certain drawbacks that in our opinion are sufficiently important to consider other options for contact. Mainly these drawbacks are:

- Impenetrability is not exactly enforced and penetration might occur.

- Small time-step size restriction.

- There are estimators for the choice of a proper penalty parameter yet there is no a common agreement for all contact conditions.

These are the reasons that motivated the study of other options, leading us to the development of a contact algorithm based on a predictor-corrector approach, that will be addressed in Section 3.4. This section will also include some benchmarks that show the performance of the algorithm. 


\section{Chapter 2}

\section{Rate dependent materials}

\subsection{Introduction}

The numerical solution of boundary value problems with solids of elasto-viscoplastic materials has been extensively studied and numerous methods already exist that, for many failure criteria, are robust and accurate both for the small and finite strain range [42]-[45]. Among these formulations, the simplest ones involve elastic domains which are convex and smooth. In these cases, viscoplastic models can be obtained simply extending the rate-independent formulations using the so-called Perzyna regularization [44], [46]. In them, the yield function itself, which is used in rate-independent plasticity to define the elastic domain, plays the role of a distance in stress space, and might be used to modulate the strength of the generalized forces that drive the stresses lying outside the elastic region towards this set.

Elasto-visco-plastic models based on yield functions that are convex, but nonsmooth, are significantly more complicated. The "distance" of a stress state from the elastic region remains as driving force, pulling it towards the yield surface as dictated by kinetic equations. In these situations, however, the yield function can no longer be used as a proxy for the distance, and Perzyna-type formulations cannot be employed. As a result, in the non-smooth case, the inviscid limit of Perzyna models does not coincide with the rate-independent theory [47], [48].

In both smooth and non-smooth viscoplastic formulations, the so-called DuvautLions viscoplastic models is valid as long as the elastic domain remains convex. In this context, the generalized forces that pull the viscoplastic stresses towards the yield surface are a function of their relative distance, the latter can be defined after obtaining the projection of the stress onto the convex yield surface, a point that is well-defined and is unique. This alternate formulation of viscoplasticity, was proposed by Duvant and Lions [49] and later numerically exploited in Simo, Ju, Pister, et al. [47] and Simo, Kennedy, and Govindjee [48]. It is in some way more complex than the Perzyna models but more general and can be applied, as indicated, to smooth and non-smooth yield functions. The complexity of the formulation has been restricted so far in all the published results to models with linear kinetic laws, a constraint that precludes modeling of more complex rate-dependent phenomena that require more that one fitting constant.

In this chapter we introduce new integration methods for viscoplastic models based on smooth and non-smooth yield criterion. Employing the ideas of the DuvautLions theory, we write the evolution problem making use of convex analysis, that allows for a holistic description of both smooth and non-smooth problems taking the geometrical concepts as a guide for the algorithmic design. Comparing with previous works on numerical modelling of viscoplasticity, the kinetic laws considered might be nonlinear, allowing for greater flexibility in their definition. To simplify the exposition we will focus on associative models with isotropic hardening. The novel 
ideas presented can also be extended to the non-associate case, but complicate the exposition of the theory.

The numerical implementation of non-smooth plastic and viscoplastic schemes is not straightforward, and especially the derivation of the consistent tangent operator [50], [51]. We consider Tresca's and Drucker-Prager's plasticity models, both non-smooth, giving the details of the implementation of the proposed integration algorithms, including the derivation of their consistent tangents. In principal stress space, Tresca's yield function is an hexagonal prism and Drucker-Prager's is a cone. In the former case the non-smoothness is concentrated in the edges of the prism and in the latter, in the apex. The generality of the results obtained provide a framework for the implementation of other smooth and non-smooth models.

In addition to fully describing the numerical implementation of the new schemes, we will show that they are unconditionally stable. With this we mean that the reduced dissipation inequality is satisfied pointwise for all converged solutions. This result holds for a wide class of elasto-visco-plastic models, based on possibly non-smooth yield functions, with nonlinear kinetic laws, making the integration algorithms particularly suitable for large scale computations.

Numerical simulations will be performed to illustrate the performance of the proposed schemes for elasto-visco-plastic problems. In them, finite element solutions are obtained for standard benchmarks using material models based on Tresca's and Drucker-Prager's yield functions.

An outline of the chapter is as follows: In section 2.2 we set the theory for smooth and non-smooth elasto-visco-plasticity, focusing on the thermodynamic framework and the geometrical viewpoint of general models. In section 2.3 we will develop integration algorithms for the abstract models described before, proving a dissipation stability estimate for all of them. Their specific implementation for the case of Tresca's and Drucker-Prager's yield function is investigated in section 2.4. where numerical examples are also provided, illustrating the behaviour of the algorithms. The main results of the chapter are summarized in section 2.5. For completeness, the detailed derivation of the consistent tangent tensor for the two models presented in section 2.4 is given in the appendix.

\subsection{Plasticity and viscoplasticity models for non-smooth yield criteria}

We will introduce this section by summarizing the basic equations of plasticity and viscoplasticity for small strain kinematics. The equations are shown in a way that they are valid both for smooth and non-smooth models. For that, we will make extensive use of concepts of convex geometry and analysis as presented, for instance, in the monograph by Han and Reddy [52].

\subsubsection{Thermodynamic setting}

In this work we put our focus on the constitutive response of a single material point on a deformable solid. In particular, isothermal elastoplastic and viscoplastic materials are described, first, by a stored energy function $\psi$ of the form

$$
\psi=\psi\left(\varepsilon-\varepsilon^{p},\{\chi\}\right),
$$

where $\varepsilon$ is the tensor of infinitesimal strain, $\varepsilon^{p}$ is the inelastic strain, and $\{\chi\}$ is an array of hardening variables. Both $\varepsilon$ and $\varepsilon^{p}$ belong to $\mathbb{S}^{3 \times 3}$, the space of symmetric 
$3 \times 3$ tensors. In what follows, and for concreteness, we will limit our exposition to the case where $\{\chi\}=\{\xi\}$ with $\xi \in[0, \infty)$ being the isotropic hardening. Moreover, we assume that the stored energy function splits as in

$$
\psi=\psi^{e}\left(\varepsilon-\varepsilon^{p}\right)+\psi^{h}(\xi)
$$

with $\psi^{e}$ modeling the energy stored in the elastic part of the deformation and $\psi^{h}$, the energy stored by work hardening. Under these assumptions, the stress tensor and the thermodynamic conjugates of the internal variables (thermodynamic forces) are defined as:

$$
\boldsymbol{\sigma}=\frac{\partial \psi}{\partial \boldsymbol{\varepsilon}}=\frac{\partial \psi^{e}}{\partial \boldsymbol{\varepsilon}}, \quad q=-\frac{\partial \psi}{\partial \xi}=-\frac{\partial \psi^{h}}{\partial \xi} .
$$

For convenience, we define the (generalized) stress as

$$
\boldsymbol{\Sigma}=(\boldsymbol{\sigma}, q)
$$

with $\boldsymbol{\Sigma} \in \mathcal{S}=\left\{\mathbb{S}^{3 \times 3} \times \mathbb{R}\right\}$, as well as the generalized total and inelastic strains as

$$
\boldsymbol{E}=(\varepsilon, 0), \quad \boldsymbol{E}^{p}=\left(\varepsilon^{p}, \xi\right) .
$$

A standard calculation shows that at every instant of any motion, the dissipation at the material point under study can be calculated as

$$
\mathcal{D}=\boldsymbol{\Sigma} \cdot \dot{\boldsymbol{E}}-\dot{\psi}=\boldsymbol{\Sigma} \cdot \dot{\boldsymbol{E}}^{p}=\boldsymbol{\sigma} \cdot \dot{\boldsymbol{\varepsilon}}^{p}+q \cdot \dot{\xi},
$$

where we have used a dot to indicate the inner product between tensors or scalars of the same order and the notation $\dot{f}$ to indicate the time derivative of any scalar, vector, or tensor function $f$.

The second law of thermodynamics implies that the dissipation $\mathcal{D}$ must be nonnegative for arbitrary processes. A simple and convenient way to enforce this constraint is to use models for which there exists a convex kinetic potential $\Xi=\Xi(\boldsymbol{\Sigma})$ such that

$$
\dot{\boldsymbol{E}}^{p} \in \partial \Xi(\boldsymbol{\Sigma})
$$

where $\partial$ indicates the sub-differential operator to cope with non-differentiable functions [see, e.g., 52]. This approach will be exploited below to formulate thermodynamically consistent models that comply with the second law of thermodynamics.

An additional ingredient of plasticity and viscoplasticity theories is the existence of a certain convex, although not necessarily smooth, closed set $\mathbb{E} \subset \mathcal{S}$, containing the zero stress, and referred to as the elastic domain. In plasticity models, the stress state $\boldsymbol{\Sigma}$ is constrained at all times to the closure of $\mathbb{E}$; in viscoplasticy models, however, the stress state can lie inside or outside of the closure of $\mathbb{E}$, but there will be a "force" driving it towards the elastic domain which increases with the "distance" between the stress state and $\mathbb{E}$. A proper way to describe the elastic domain is by means of a function $F: \mathcal{S} \rightarrow \mathbb{R}$, the yield function, such that

$$
\mathbb{E}=\{\boldsymbol{\Sigma}, F(\boldsymbol{\Sigma}) \leq 0\} .
$$

For rate-independent plasticity, in particular, the kinetic potential can be chosen to be $\Xi=F$, in which case the model is called associative. This choice is very convenient from the modeling and computation standpoints because it reduces the amount of parameters to be fitted. From the theoretical point of view, in addition, it guarantees that for a given stress state, the strain rate is always such that the dissipation (3.53) 
is maximum [52].

To close this section, we mention that often the stored energy function is chosen to be of the form

$$
\psi=\frac{1}{2}\left(\boldsymbol{E}-\boldsymbol{E}^{p}\right) \cdot \mathbb{G} \cdot\left(\boldsymbol{E}-\boldsymbol{E}^{p}\right),
$$

where $\mathbb{G}$ is a constant, block-diagonal matrix of the form

$$
\mathbb{G}=\operatorname{DIAG}\left[\mathbb{C}, H_{i s o}\right] .
$$

In this expression, $\mathbb{C}$ and $H_{i s o}$ are, respectively, the fourth order tensors of elasticities and isotropic hardening modulus. If, in particular, the elastic response is isotropic, the tensor $\mathbb{C}$ can be expressed as

$$
\mathbb{C}=\kappa \boldsymbol{I} \otimes \boldsymbol{I}+2 \mu \mathbb{I}^{\text {dev }}
$$

with $\boldsymbol{I}$ being the second order identity tensor, $\otimes$ the dyadic product operator, $\mathbb{I}^{\text {dev }}$ the identity fourth order tensor on symmetric deviatoric tensors, and $\kappa, \mu$ the bulk and shear moduli, respectively. We assume the hardening modulus to be constant and positive. For a non-hardening model we could just use a model with zero hardening modulus, making $\xi$ to play no role at all. In this case, all the derivations of the article would simplify, remaining essentially identical.

\subsubsection{Plasticity}

As mentioned in the previous section, the main feature of classical, rate-independent plasticity models is that they constrain the stress state $\boldsymbol{\Sigma}$ to lie within the closure of $\mathbb{E}$. In particular, associative plasticity defines the kinetic potential $\Xi$, that drives the evolution of the internal variables, to be the indicator function of the elastic domain, denoted $I_{\mathbb{E}}$, and thus the evolution equations of the internal variables become simply

$$
\dot{\boldsymbol{E}}^{p} \in \partial I_{\mathbb{E}}(\boldsymbol{\Sigma}) \equiv N_{\mathbb{E}}(\boldsymbol{\Sigma})
$$

where $N_{\mathbb{E}}$ is the normal cone to the elastic domain at $\boldsymbol{\Sigma}$. The formulation of the evolution of internal variables as in Eq. (2.12) has the advantage that unifies all possible flow rules, including in particular those derived from non-smooth elastic regions.

Let us define the dissipation $\Delta$ as the function on the plastic strain rates given by

$$
\Delta\left(\dot{\boldsymbol{E}}^{p}\right)=\max _{\boldsymbol{T} \in \mathbb{E}} \boldsymbol{T} \cdot \dot{\boldsymbol{E}}^{p} .
$$

It follows that this function is the Legendre dual of $I_{\mathbb{E}}$ and thus, by duality, Eq. (2.12) is equivalent to

$$
\boldsymbol{\Sigma} \in \partial \Delta\left(\dot{\boldsymbol{E}}^{p}\right)
$$

which is an abstract statement of the principle of maximum dissipation.

When the elastic region is given as in Eq. (2.8), the flow rule (2.12) can be written as

$$
\dot{\boldsymbol{E}}^{p} \in \dot{\lambda} \partial_{\boldsymbol{\Sigma}} F(\boldsymbol{\Sigma}),
$$

where $\dot{\lambda} \geq 0$ is referred to as the plastic multiplier. In certain applications, it might be more convenient to move away from the associative framework and choose a dissipation potential $\phi \neq I_{\mathbb{E}}$, which could be non-smooth as well. Then, the flow of the inelastic variables (2.12) is replaced by the alternative

$$
\dot{\boldsymbol{E}}^{p} \in \dot{\lambda} \partial_{\boldsymbol{\Sigma}} \phi(\boldsymbol{\Sigma}) .
$$


This latter formulation gives more flexibility to the evolution equations, at the expense of loosing some of the duality relations identified before and giving a constitutive behaviour that does not verify the principle of maximum dissipation. In both the associated and non-associated cases, the subdifferential of the kinetic potential defines the admissible directions for the plastic flow.

\subsubsection{Viscoplasticity}

We now extend next the concepts of the previous section to the viscoplastic case, whose main difference with the rate independent or so-called inviscid theory is that the generalized stress $\Sigma$ can now lie outside the elastic region. When this is the case, classical viscoplasticity theory states that there is a "force" driving the stress state onto the yield surface. In general the greater the force, the farther the stress state is from the elastic surface. Different models replace the flow rule (2.12) with a kinetic equation that defines the strength and direction of this "force" based on how far the stress is from the elastic region.

A simple way to formulate a viscoplastic kinetic equation is to use Perzyna-type models [46]. In these models, the scalar $F(\boldsymbol{\Sigma})$ is used as a measure of the distance of $\boldsymbol{\Sigma}$ to the elastic region and the direction of the force driving the stress state to $\mathbb{E}$ is proportional to the gradient of $F$. More precisely, given a viscosity $\eta$, the evolution equation for the inelastic strains is given by

$$
\dot{\boldsymbol{E}}^{p}=\frac{\langle F(\boldsymbol{\Sigma})\rangle}{\eta} \partial_{\boldsymbol{\Sigma}} F(\boldsymbol{\Sigma})
$$

where $\langle\cdot\rangle$ is the Macaulay bracket. The Perzyna model can be also obtained by regularizing the rate-independent problem where the plastic multiplier is replaced by $\langle F(\boldsymbol{\Sigma})\rangle / \eta$.

Perzyna's formulation yields a straightforward extension of the rate-independent theory and thus it has been often employed for the formulation of numerical methods [42], [44]. Its main disadvantage is that relies on the fact that the yield function $F$ must define a distance function from points in stress space to the elastic domain. In classical von Mises plasticity, for example, this is the case. In so-called multi-surface plasticity, however, it can be easily shown that the Perzyna model predicts an incorrect inviscid limit due precisely to the non-smoothness of the elastic domain, which in turn implies that there exist regions in the space of generalized stresses where the yield function does not define correctly the distance to the elastic domain [48].

A viscoplastic model could be formulated for arbitrary elastic domains provided a distance function to this set were known explicitly. However, since the elastic domain is convex, by assumption, the distance function can always be defined. Assuming that the generalized stress space has a norm \|\|$\cdot\|\|: \mathcal{S} \rightarrow[0, \infty)$, the distance of a generalized stress $\boldsymbol{\Sigma}$ to $\mathbb{E}$ can always be defined to be

$$
d_{\mathbb{E}}(\boldsymbol{\Sigma})=\min _{\boldsymbol{T} \in \mathbb{E}}|\|\boldsymbol{\Sigma}-\boldsymbol{T} \mid\| .
$$

A convenient norm in stress space is afforded by the generalized moduli $\mathbb{G}$. More precisely, the norm

$$
\||\boldsymbol{T}|\|=\left(\boldsymbol{T} \cdot \tilde{\mathbb{G}}^{-1} \boldsymbol{T}\right)^{1 / 2}
$$

can be used to define the distance employed in Eq. (2.18), where we have introduced the nondimensional moduli

$$
\tilde{\mathbb{G}}=\delta^{-1} \mathbb{G},
$$


and $\delta$ is some characteristic stiffness of the material, such as Young's modulus of the elastic response. Using the notion of distance to a set, the unique closest projection of a stress state onto the elastic region can be defined as

$$
\overline{\boldsymbol{\Sigma}}=\underset{\boldsymbol{T} \in \mathbb{E}}{\arg \min }|\|\boldsymbol{\Sigma}-\boldsymbol{T}\||
$$

Duvaut-Lions viscoplastic models employ these ideas and define formulations that are more general than Perzyna's formulation. For smooth or non-smooth elastic domains, viscoplastic kinetic models can always be defined to be of the form

$$
\dot{\boldsymbol{E}}^{p}=\frac{1}{\tau} \mathbb{G}^{-1}(\boldsymbol{\Sigma}-\overline{\boldsymbol{\Sigma}})
$$

where $\tau$ is a characteristic relaxation time. In view of the uniqueness of $\bar{\Sigma}$ for smooth or non-smooth yield surfaces, Eq. (2.22) is always well-defined, thereby making Duvaut-Lions a more general approach for viscoplasticity.

In fact, Duvaut-Lions viscoplasticity can be conveniently recast using the concepts of Section 2.2.1. For that, consider the kinetic potential

$$
\Xi(\boldsymbol{\Sigma})=\frac{d_{\mathbb{E}}^{2}(\boldsymbol{\Sigma})}{2 \tau \delta}
$$

with $d_{\mathbb{E}}$ being the distance function defined in Eq. (2.18). The subdifferential of the distance to a convex set is always well-defined [53], and can be evaluated to be

$$
\partial d_{\mathbb{E}}(\boldsymbol{\Sigma})= \begin{cases}\frac{1}{d_{\mathbb{E}}(\boldsymbol{\Sigma})} \tilde{\mathbb{G}}^{-1}(\boldsymbol{\Sigma}-\overline{\boldsymbol{\Sigma}}), & \text { when } d_{\mathbb{E}}(\boldsymbol{\Sigma})>0 \\ \emptyset & \text { when } d_{\mathbb{E}}(\boldsymbol{\Sigma})=0\end{cases}
$$

hence it follows that the general evolution equation (2.7) corresponds, in the present case, to Eq. 2.22). In fact, for any differentiable, convex function $J: \mathbb{R}^{+} \cup\{0\} \rightarrow \mathbb{R}$ with $J(0)=0$ one could consider the more general class of potentials

$$
\Xi(\boldsymbol{\Sigma})=\frac{\delta}{\tau} J\left(d_{\mathbb{E}}(\boldsymbol{\Sigma}) / \delta\right)
$$

leading to the evolution equation

$$
\dot{\boldsymbol{E}}^{p}=\frac{1}{\tau d_{\mathbb{E}}(\boldsymbol{\Sigma})} J^{\prime}\left(d_{\mathbb{E}}(\boldsymbol{\Sigma}) / \delta\right) \tilde{\mathbb{G}}^{-1}(\boldsymbol{\Sigma}-\overline{\boldsymbol{\Sigma}}),
$$

with the limit case $\dot{\boldsymbol{E}}^{p}=0$ when $d_{\mathbb{E}} \boldsymbol{\Sigma}=0$. The choice $J(x)=x^{2} / 2$ reduces the previous expression to Eq. (2.22).

The potential (2.25) defines, via Eq. (2.7), a general class of kinetic equations of the Duvaut-Lions type suitable for models defined on non-smooth elastic domains, such as Drucker-Prager's. Moreover, since the function $\Xi$ is convex, the mechanical dissipation is guaranteed to be non-negative for all of them.

The specific modelling of the viscoplastic effects reduces to the choice of the characteristic time $\tau$ and the functional form of the viscous response, i.e., the function $J$. This framework gives the theory much more flexibility than the simple linear model (2.22). Thus, endowing it with a wide variety of behaviors.

Finally, we note that if we take the norm in stress space to be $\|\boldsymbol{T}\|\|=\| \boldsymbol{T} \|$, i.e., the Euclidean norm, then $\tilde{\mathbb{G}}$ becomes the identity rank-four tensor and for $J(x)=x^{2} / 2$ 
the choice Eq. 2.26) reduces to

$$
\dot{\boldsymbol{E}}^{p}=\frac{1}{\tau \delta}(\boldsymbol{\Sigma}-\overline{\boldsymbol{\Sigma}})
$$

In the event that the yield function $F$ provides a true distance to the elastic domain, i.e., $d_{\mathbb{E}}(\boldsymbol{\Sigma})=F(\boldsymbol{\Sigma})$, and we define the viscosity $\eta=\tau \delta$, then Eqs. (2.17) and (2.27) coincide, which shows the equivalence between Perzyna's and Duvaut-Lions' formulations for viscoplasticity.

\subsection{Integration algorithms for general elasto-visco-plasticity}

In this section we describe the algorithmic discretization of the general viscoplastic differential equation (2.26). In particular, we identify the close relationship with the rate independent solution, its abstract geometric interpretation and finally we provide the major lines for its solution. Remarkably, the smoothness of the elastic domain is not required for any of the results presented, giving the possibility of specific implementations based on, for example, Druker-Prager's or Tresca's yield criteria.

\subsubsection{General structure}

As in standard numerical implementations for plasticity and viscoplasticity, we formulate next a strain driven method for the integration of Eq. (2.26). For that, we assume that the generalized total and inelastic strains at time $t_{n}$, denoted as $\boldsymbol{E}_{n}=$ $\left(\varepsilon_{n}, 0\right)$ and $\boldsymbol{E}_{n}^{p}=\left(\varepsilon_{n}^{p}, \xi_{n}\right)$, and the total strain at time $t_{n+1}=t_{n}+\Delta t$, denoted $\boldsymbol{E}_{n+1}=\left(\varepsilon_{n+1}, 0\right)$ are known. Here, and below, $\Delta t$ refers to the time step size, which is considered for simplicity to be independent of $n$. The goal of the integration algorithm is to compute $\boldsymbol{\Sigma}_{n+1}=\left(\boldsymbol{\sigma}_{n+1}, q_{n+1}\right)$, the generalized stress at time $t_{n+1}$, and update the inelastic strains $\boldsymbol{E}_{n+1}^{p}$.

For that, we propose to use a Backward-Euler approximation of Eq. 2.26, which reads

$$
\frac{\boldsymbol{E}_{n+1}^{p}-\boldsymbol{E}_{n}^{p}}{\Delta t}=\frac{J^{\prime}\left(d_{\mathbb{E}}\left(\boldsymbol{\Sigma}_{n+1}\right) / \delta\right)}{\tau d_{\mathbb{E}}\left(\boldsymbol{\Sigma}_{n+1}\right)} \widetilde{\mathbb{G}}^{-1}\left(\boldsymbol{\Sigma}_{n+1}-\overline{\boldsymbol{\Sigma}}_{n+1}\right),
$$

together with the elastic relations

$$
\boldsymbol{\Sigma}_{n+1}=\mathbb{G}\left(\boldsymbol{E}_{n+1}-\boldsymbol{E}_{n+1}^{p}\right) .
$$

Eqs. (2.28) and (2.29) define an implicit system of equations whose solution will be addressed next. The main challenge for its solution is the computation of $\overline{\boldsymbol{\Sigma}}_{n+1}$, that is also required for the evaluation of the distance, since it depends, in general, on the solution of the projection (2.21) which may or may not be available in closed form, depending on the form of $\mathbb{E}$.

In order to integrate the update equations, we use a predictor-corrector strategy, as it is commonly done in computational inelasticity [see, e.g. 44]. First, we define the so-called trial stress state $\boldsymbol{\Sigma}_{n+1}^{t r}$ which would be the stress at time $t_{n+1}$ if the strain increment from $t_{n}$ to $t_{n+1}$ were purely elastic, i.e.,

$$
\boldsymbol{\Sigma}_{n+1}^{t r}=\mathbb{G}\left(\boldsymbol{E}_{n+1}-\boldsymbol{E}_{n}^{p}\right) .
$$


Using this definition and Eq. (2.29), the update (2.28) can be re-written as

$$
\boldsymbol{\Sigma}_{n+1}^{t r}-\boldsymbol{\Sigma}_{n+1}=\frac{\Delta t \delta J^{\prime}\left(d_{\mathbb{E}}\left(\boldsymbol{\Sigma}_{n+1}\right) / \delta\right)}{\tau d_{\mathbb{E}}\left(\boldsymbol{\Sigma}_{n+1}\right)}\left(\boldsymbol{\Sigma}_{n+1}-\overline{\boldsymbol{\Sigma}}_{n+1}\right),
$$

\subsubsection{The abstract rate-independent problem}

Before focusing on the study of the solution to the elasto-viscoplastic evolution equation, we consider the related topic of solving numerically the rate independent limit of the viscoplastic problem.

In plasticity, the flow rule is given by Eq. (2.12). Its numerical solution follows the same steps described for rate dependent viscoplasticity: with the known state $\boldsymbol{E}_{n}, \boldsymbol{E}_{n}^{p}$, and given a new elastic strain $\boldsymbol{E}_{n+1}$ the goal is to calculate $\boldsymbol{E}_{n+1}^{p}$ that solves

$$
\frac{\boldsymbol{E}_{n+1}^{p}-\boldsymbol{E}_{n}^{p}}{\Delta t} \in \partial I_{\mathbb{E}}\left(\boldsymbol{\Sigma}_{n+1}\right)
$$

where $\boldsymbol{\Sigma}_{n+1}$ is given by relation (2.29). Due to the nature of the indicator function, the generalized stress is bound to remain in the closure of the elastic domain.

The solution of Eq. (2.32) can be obtained using, as done before, a predictorcorrector scheme based on the Backward-Euler integration method and the notion of trial state $\boldsymbol{\Sigma}_{n+1}^{t r}$ defined in Eq. (2.30). If the trial stress is within $\mathbb{E}$, then the solution $\boldsymbol{E}_{n+1}^{p} \equiv \boldsymbol{E}_{n}^{p}$, which implies $\boldsymbol{\Sigma}_{n+1} \equiv \boldsymbol{\Sigma}_{n+1}^{t r}$ satisfies Eq. (2.32) since $\partial I_{\mathbb{E}}\left(\boldsymbol{\Sigma}_{n+1}\right)=\emptyset$. On the other hand, if $\boldsymbol{\Sigma}_{n+1}^{t r}$ lies outside the elastic domain, the stress $\boldsymbol{\Sigma}_{n+1}$ can not be equal to the trial one, the plastic strain $\boldsymbol{E}_{n+1}^{p}$ must be different to $\boldsymbol{E}_{n}^{p}$, and $\boldsymbol{\Sigma}_{n+1}$ must belong to the boundary of $\mathbb{E}$ so that Eq. (2.32) is satisfied.

Postponing for the moment the actual solution of the elastoplastic problem, we recall next a classical interpretation of the numerical solution of rate-independent plasticity: if $\mathbb{E}$ is convex, the stress $\boldsymbol{\Sigma}_{n+1}$ is the closest projection onto the elastic region of $\boldsymbol{\Sigma}_{n+1}^{t r}$ with respect to the norm 2.19). Denoting by $\overline{\boldsymbol{\Sigma}}_{n+1}^{t r}$ the projection of $\Sigma_{n+1}^{t r}$ onto $\mathbb{E}$, we note that

$$
\overline{\boldsymbol{\Sigma}}_{n+1}^{t r}=\underset{\boldsymbol{T} \in \boldsymbol{E}}{\arg \min } \frac{1}{2}\left\|\left|\boldsymbol{\Sigma}_{n+1}^{t r}-\boldsymbol{T}\right|\right\|^{2}=\underset{\boldsymbol{T} \in \mathcal{S}}{\arg \min } \frac{1}{2}\left\|\left|\boldsymbol{\Sigma}_{n+1}^{t r}-\boldsymbol{T} \|\right|^{2}+I_{\mathbb{E}}(\boldsymbol{T}),\right.
$$

and thus

$$
\mathbf{0} \in \tilde{\mathbb{G}}^{-1}\left(\boldsymbol{\Sigma}_{n+1}^{t r}-\overline{\boldsymbol{\Sigma}}_{n+1}^{t r}\right)+\partial I_{\mathbb{E}}\left(\overline{\boldsymbol{\Sigma}}_{n+1}^{t r}\right) .
$$

Re-writing Eq. (2.32) as

$$
\begin{aligned}
\partial I_{\mathbb{E}}\left(\boldsymbol{\Sigma}_{n+1}\right) & \ni \mathbb{G}^{-1}\left(\mathbb{G}\left(\boldsymbol{E}_{n+1}-\boldsymbol{E}_{n}^{p}\right)+\mathbb{G}\left(\boldsymbol{E}_{n+1}^{p}-\boldsymbol{E}_{n+1}\right)\right) \\
& =\mathbb{G}^{-1}\left(\boldsymbol{\Sigma}_{n+1}^{t r}-\boldsymbol{\Sigma}_{n+1}\right),
\end{aligned}
$$

and comparing it with Eq. 2.33), we conclude that $\overline{\boldsymbol{\Sigma}}_{n+1}^{t r}=\boldsymbol{\Sigma}_{n+1}$. Remarkably, this result does not require any smoothness in $\mathbb{E}$.

\subsubsection{The projection problem}

The solution of Eq. (2.31) for $\boldsymbol{\Sigma}_{n+1}$ is not straightforward because it involves the solution of the projected stress $\overline{\boldsymbol{\Sigma}}_{n+1}$ and the non-smooth function $d_{\mathbb{E}}\left(\boldsymbol{\Sigma}_{n+1}\right)$. Nevertheless, it is evident from this equation that the three generalized stresses $\boldsymbol{\Sigma}_{n+1}, \overline{\boldsymbol{\Sigma}}_{n+1}, \boldsymbol{\Sigma}_{n+1}^{t r}$ are colinear. Using only this fact and the convexity of the elastic region we can prove the following 
Theorem 1. Let $\boldsymbol{\Sigma}_{n+1}, \boldsymbol{\Sigma}_{n+1}^{t r}$ be two generalized stresses outside of a convex elastic region $\mathbb{E}$ and let $\overline{\boldsymbol{\Sigma}}_{n+1}$ be the closest projection of $\boldsymbol{\Sigma}_{n+1}$ onto $\mathbb{E}$ with respect to the norm defined in Eq. 2.19) with $\tilde{\mathbb{G}}$ constant. Then, if $\boldsymbol{\Sigma}_{n+1}, \overline{\boldsymbol{\Sigma}}_{n+1}, \boldsymbol{\Sigma}_{n+1}^{t r}$ are colinear, the projection of $\boldsymbol{\Sigma}_{n+1}^{t r}$ onto $\mathbb{E}$, which is denoted $\overline{\mathbf{\Sigma}}_{n+1}^{t r}$ is equal to $\overline{\mathbf{\Sigma}}_{n+1}$.

Proof. The projected stress is defined as

$$
\overline{\boldsymbol{\Sigma}}_{n+1}=\left.\underset{\boldsymbol{T} \in \mathbb{E}}{\arg \min } \frac{1}{2}\left\|\left|\boldsymbol{\Sigma}_{n+1}-\boldsymbol{T}\left\|\left.\right|^{2}=\underset{\boldsymbol{T} \in \mathcal{S}}{\arg \min } \frac{1}{2}\right\|\right| \boldsymbol{\Sigma}_{n+1}-\boldsymbol{T}\right\|\right|^{2}+I_{\mathbb{E}}(\boldsymbol{T}) .
$$

Hence, for any convex set $\mathbb{E}$,

$$
\mathbf{0} \in \widetilde{\mathbb{G}}^{-1}\left(\boldsymbol{\Sigma}_{n+1}-\overline{\boldsymbol{\Sigma}}_{n+1}\right)+\partial I_{\mathbb{E}}\left(\overline{\boldsymbol{\Sigma}}_{n+1}\right) .
$$

But, since $\boldsymbol{\Sigma}_{n+1}, \overline{\boldsymbol{\Sigma}}_{n+1}, \boldsymbol{\Sigma}_{n+1}^{t r}$ are colinear, $\boldsymbol{\Sigma}_{n+1}-\overline{\boldsymbol{\Sigma}}_{n+1}$ is proportional to $\boldsymbol{\Sigma}^{t r}-\overline{\boldsymbol{\Sigma}}_{n+1}$. Thus, since $\partial I_{\mathbb{E}}\left(\overline{\boldsymbol{\Sigma}}_{n+1}\right)$ is a cone, $\alpha \partial I_{\mathbb{E}}\left(\overline{\boldsymbol{\Sigma}}_{n+1}\right)=\partial I_{\mathbb{E}}\left(\overline{\boldsymbol{\Sigma}}_{n+1}\right)$ when $\alpha>0$, and it follows that

$$
\mathbf{0} \in \tilde{\mathbb{G}}^{-1}\left(\boldsymbol{\Sigma}_{n+1}^{t r}-\overline{\boldsymbol{\Sigma}}_{n+1}\right)+\partial I_{\mathbb{E}}\left(\overline{\boldsymbol{\Sigma}}_{n+1}\right) .
$$

But this means that $\overline{\boldsymbol{\Sigma}}_{n+1}$ is the projection of $\boldsymbol{\Sigma}_{n+1}^{t r}$ onto $\mathbb{E}$, i.e., $\overline{\boldsymbol{\Sigma}}_{n+1}^{t r}=\overline{\boldsymbol{\Sigma}}_{n+1}$.

As said before, the importance of this result is that it holds irrespective of the smoothness of the elastic domain, and for every projection, not only those onto the smooth parts of $\mathbb{E}$. It should be noted that the hypotheses of the theorem include the collinearity of the generalized stresses. In particular, the collinearity of the stress tensors themselves do not suffice to guarantee that the projections $\bar{\sigma}$ and $\bar{\sigma}^{t r}$ are equal. It bears emphasis that the result, while trivially true for projections in Euclidean space, is shown to hold in stress space as long as the metric is constant.

Since for this result to hold the smoothness of $\mathbb{E}$ is not required, it follows that, for every viscoplastic model, as long as the hypotheses of Theorem 1 hold, Eq. (2.31) can be replaced with

$$
\boldsymbol{\Sigma}_{n+1}^{t r}-\boldsymbol{\Sigma}_{n+1}=\frac{\Delta t \delta J^{\prime}\left(d_{\mathbb{E}}\left(\boldsymbol{\Sigma}_{n+1}\right) / \delta\right)}{\tau d_{\mathbb{E}}\left(\boldsymbol{\Sigma}_{n+1}\right)}\left(\boldsymbol{\Sigma}_{n+1}-\overline{\boldsymbol{\Sigma}}_{n+1}^{t r}\right),
$$

From the algorithmic viewpoint, Eq. (2.39) is much easier to solve then Eq. (2.31) because $\boldsymbol{\Sigma}_{n+1}^{t r}$ is known beforehand, $\overline{\boldsymbol{\Sigma}}_{n+1}^{t r}$ is readily obtained, and the only remaining unknown is $\boldsymbol{\Sigma}_{n+1}$. We remark that this result requires that the norm $\|\Perp|\||$ uses a constant metric $\tilde{\mathbb{G}}$ or, equivalently, that the stored energy function 2.9 ) is quadratic. In fact, as shown before, $\overline{\boldsymbol{\Sigma}}_{n+1}^{t r}$ is the rate-independent solution for the data $\boldsymbol{E}_{n}^{p}, \boldsymbol{E}_{n+1}$.

The structure of the update (2.39) can be exploited to reduce the problem to the solution of a nonlinear scalar equation. To see this, first let us rewrite Eq. (2.39) as

$$
\boldsymbol{\Sigma}_{n+1}^{t r}-\overline{\boldsymbol{\Sigma}}_{n+1}^{t r}=\left(1+\frac{\Delta t}{\tau} \frac{\delta J^{\prime}\left(d_{\mathbb{E}}\left(\boldsymbol{\Sigma}_{n+1}\right) / \delta\right)}{d_{\mathbb{E}}\left(\boldsymbol{\Sigma}_{n+1}\right)}\right)\left(\boldsymbol{\Sigma}_{n+1}-\overline{\boldsymbol{\Sigma}}_{n+1}^{t r}\right) .
$$

Then, taking norms of both sides of the identity we get

$$
d_{\mathbb{E}}\left(\boldsymbol{\Sigma}_{n+1}^{t r}\right)=\left(1+\frac{\Delta t}{\tau} \frac{\delta J^{\prime}\left(d_{\mathbb{E}}\left(\boldsymbol{\Sigma}_{n+1}\right) / \delta\right)}{d_{\mathbb{E}}\left(\boldsymbol{\Sigma}_{n+1}\right)}\right) d_{\mathbb{E}}\left(\boldsymbol{\Sigma}_{n+1}\right),
$$

whose only unknown quantity is the distance $d_{\mathbb{E}}\left(\boldsymbol{\Sigma}_{n+1}\right)$. Once it is obtained, the update of the stress $\boldsymbol{\Sigma}_{n+1}$ follows trivially from Eq. (2.40). 


\subsubsection{Local stability estimate}

Continuum models of dissipative materials should satisfy the reduced dissipation inequality $\mathcal{D}=\boldsymbol{\Sigma} \cdot \dot{\boldsymbol{E}}^{p} \geq 0$. The viscoplastic models formulated in this section naturally inherit this stability property and strictly satisfy

$$
\boldsymbol{\Sigma}_{n+1} \cdot \frac{\boldsymbol{E}_{n+1}^{p}-\boldsymbol{E}_{n}^{p}}{\Delta t} \geq 0 .
$$

To prove this, simply note that Eq. 2.28) can be written as

$$
\frac{\boldsymbol{E}_{n+1}^{p}-\boldsymbol{E}_{n}^{p}}{\Delta t}=\partial_{\boldsymbol{\Sigma}_{n+1}}\left(\frac{\delta}{\tau} J\left(d_{\mathbb{E}}\left(\boldsymbol{\Sigma}_{n+1}\right) / \delta\right)\right)=\partial_{\boldsymbol{\Sigma}_{n+1}} \Xi\left(\boldsymbol{\Sigma}_{n+1}\right),
$$

Hence,

$$
\boldsymbol{\Sigma}_{n+1} \cdot \frac{\boldsymbol{E}_{n+1}^{p}-\boldsymbol{E}_{n}^{p}}{\Delta t}=\boldsymbol{\Sigma}_{n+1} \cdot \partial_{\boldsymbol{\Sigma}_{n+1}} \Xi\left(\boldsymbol{\Sigma}_{n+1}\right) \geq 0
$$

due to the convexity of the function $\Xi$.

\subsection{Applications}

The results shown in Section 2.3 are general and describe integration algorithms for a broad class of small-strain viscoplastic models. To show the performance of the presented approach, we consider next their application to the integration of two well-known, viscoplastic models based on non-smooth yield surfaces with linear isotropic hardening, namely, Tresca's and Drucker-Prager's. As stated before the addition of kinematic hardening poses no additional difficulties.

Summarizing the results of Section 2.3, the integration of viscoplastic models follows always the same steps: Given $\boldsymbol{E}_{n}, \boldsymbol{E}_{n}^{p}$, and $\boldsymbol{E}_{n+1}$,

1) Calculate the trial state $\boldsymbol{\Sigma}_{n+1}^{t r}=\mathbb{G}\left(\boldsymbol{E}_{n+1}-\boldsymbol{E}_{n}^{p}\right)$.

2) If $F\left(\boldsymbol{\Sigma}_{n+1}^{t r}\right) \leq 0$ then $\boldsymbol{\Sigma}_{n+1}=\boldsymbol{\Sigma}_{n+1}^{t r}$ and $\boldsymbol{E}_{n+1}^{p}=\boldsymbol{E}_{n}^{p}$, and the update is finished.

3) Else,

a) Project the trial stress onto the yield surface $\boldsymbol{\Sigma}_{n+1}^{t r} \mapsto \overline{\boldsymbol{\Sigma}}_{n+1}^{t r}$.

b) Solve for the distance $d_{\mathbb{E}}\left(\boldsymbol{\Sigma}_{n+1}\right)$ using the nonlinear scalar equation (2.41), namely,

$$
d_{\mathbb{E}}\left(\boldsymbol{\Sigma}_{n+1}^{t r}\right)=\left(1+\frac{\Delta t}{\tau} \frac{\delta J^{\prime}\left(d_{\mathbb{E}}\left(\boldsymbol{\Sigma}_{n+1}\right) / \delta\right)}{d_{\mathbb{E}}\left(\boldsymbol{\Sigma}_{n+1}\right)}\right) d_{\mathbb{E}}\left(\boldsymbol{\Sigma}_{n+1}\right) .
$$

c) Solve for $\Sigma_{n+1}$ employing Eq. 2.40, giving

$$
\boldsymbol{\Sigma}_{n+1}=\overline{\boldsymbol{\Sigma}}_{n+1}^{t r}+\left(1+\frac{\Delta t}{\tau} \frac{\delta J^{\prime}\left(d_{\mathbb{E}}\left(\boldsymbol{\Sigma}_{n+1}\right) / \delta\right)}{d_{\mathbb{E}}\left(\boldsymbol{\Sigma}_{n+1}\right)}\right)^{-1}\left(\boldsymbol{\Sigma}_{n+1}^{t r}-\overline{\boldsymbol{\Sigma}}_{n+1}^{t r}\right)
$$

d) Update the generalized plastic strain

$$
\boldsymbol{E}_{n+1}^{p}=\boldsymbol{E}_{n+1}-\mathbb{G}^{-1} \boldsymbol{\Sigma}_{n+1} .
$$

The steps in the previous algorithm are independent of the plasticity model, except for the projection $\Sigma_{n+1}^{t r} \mapsto \bar{\Sigma}_{n+1}^{t r}$ of step 3.a which, obviously, this step is specific 

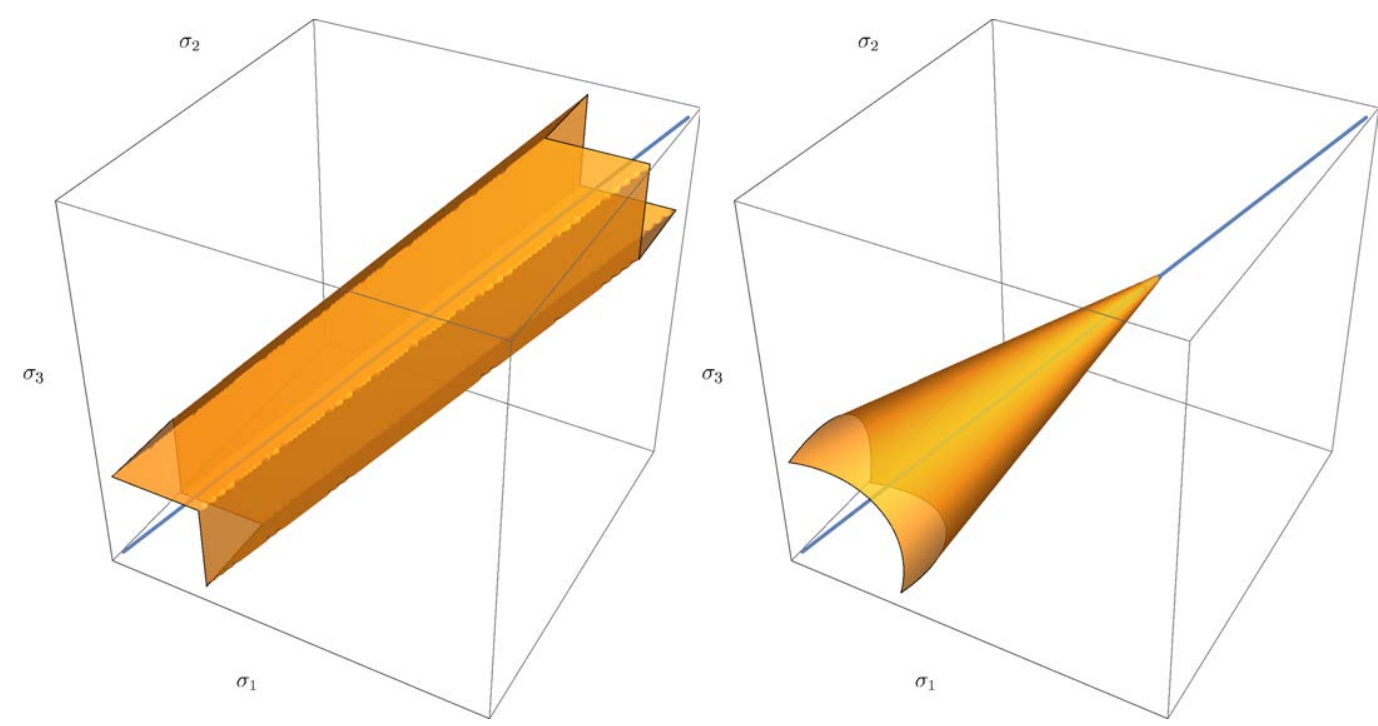

FIGURE 2.1: Tresca yield surface (left) and Drucker-Prager's (right) in the space of principal stresses for $q=0$. The axis of hydrostatic stresses is shown in blue.

for each yield surface and is in fact the inviscid solution of plasticity. We will show the performance of this integration scheme to the solution of viscoplastic models based on Tresca's and Drucker-Prager's yield criteria. For that, we review their yield functions and hint at their rate-independent solution giving, in some cases, comparisons between both the rate-dependent and rate-independent solution. Since solutions to this step are well documented in the literature, we simply provide references to existing implementations.

\subsubsection{Tresca's viscoplasticity}

Tresca's model is often used in the analysis of metal behavior. It is based on a remarkably simple yield function which, however, is non-smooth. As seen in the principal stress space its shape is an infinite hexagonal prism, see Fig. 2.1. The yield surface is non-smooth at the edges. For a material with isotropic hardening, we define as in Section 2.2 the generalized stress $\boldsymbol{\Sigma}=(\boldsymbol{\sigma}, q)$, where $\boldsymbol{\sigma}$ is the stress tensor and $q$, a scalar stress-like isotropic hardening variable. If $\left(\sigma_{1}, \sigma_{2}, \sigma_{3}\right)$ are the three principal stresses, Tresca's yield function can be written as

$$
F(\boldsymbol{\Sigma})=\max \left(\left|\sigma_{1}-\sigma_{2}\right|,\left|\sigma_{2}-\sigma_{3}\right|,\left|\sigma_{3}-\sigma_{1}\right|\right)-\left(\sigma_{0}-q\right),
$$

where $\sigma_{0}$ is the nominal yield stress. The simplicity of expression 2.48 is misleading, since the calculation of the principal stresses is a non-linear function of the argument $\boldsymbol{\Sigma}$ and the function "max" is non-smooth.

For plasticity models with isotropic hardening, the generalized inelastic strain is, according to Section 2.2, of the form $\boldsymbol{E}^{p}=\left(\varepsilon^{p}, \xi\right)$, with $\varepsilon^{p}$ being the plastic strain and $\xi$ the strain-like kinematic hardening variable. We want to limit our exposition to associative models and we will define the elastic domain $\mathbb{E}$ as in Eq. (2.8), the elastoplastic problem is closed with the flow equation for the inelastic strains, as given by Eq. (2.7).

There are different existing options for the integration of the rate-independent Tresca's model. For example, some authors choose to express the flow equations in the so-called multi-surface form [48], where the elastic domain $\mathbb{E}$ is expressed as 
the intersection of several smooth surfaces. Thus, the solution of the non-smooth problem is replaced by a sequence of solutions of smooth plasticity problems. In any approach, however, the basic problem to be solved is the projection of the trial stress $\boldsymbol{\Sigma}_{n+1}^{t r}$ onto the elastic domain $\mathbb{E}$, as indicated by Eqs. (2.33). In Souza Neto, Peric, and Owen [42], for instance, this projection is suggested to be done directly in principal stress space, using the geometrical features of the yield surface to calculate the projected stress. Since both strategies are standard, we refer to the works cited above and assume that, for Tresca's model, given $\boldsymbol{E}_{n}, \boldsymbol{E}_{n}^{p}$ and $\boldsymbol{E}_{n+1}$, the calculation of $\bar{\Sigma}_{n+1}^{t r}$, the projected trial stress, and $\mathbb{C}^{e p}$, the elastoplastic tangent, are known.

\subsubsection{Drucker-Prager's viscoplasticity}

Drucker-Prager's model is often employed to model the failure of materials, like clays, soils and ice, that might yield under deviatoric stresses but whose strength depends on the pressure. From the numerical viewpoint, its integration poses even more difficulties than Tresca's model because not only it is based on a non-smooth yield surface but this one cannot be expressed as the intersection of a finite number of smooth surfaces. Instead it is an intersection of an infinite number of planes that have and intersecting common point, the apex, where the non-smoothness appears. As a result, integration methods based on multi-surface plasticity are of no use, and the rate-independent solution has to be obtained explicitly calculating projections onto the yield surface.

In order to define the yield function of a model with isotropic hardening we define, as before, the generalized stress $\boldsymbol{\Sigma}=(\boldsymbol{\sigma}, q)$ and consider the yield function

$$
F(\boldsymbol{\Sigma})=\sqrt{J_{2}(\operatorname{dev}[\boldsymbol{\sigma}])}+\alpha \operatorname{tr}[\boldsymbol{\sigma}]-\left(\alpha+\frac{\sqrt{3}}{3}\right)\left(\sigma_{0}-q\right) .
$$

In the previous equation $\operatorname{tr}[\boldsymbol{\sigma}]=\boldsymbol{\sigma} \cdot \boldsymbol{I}$ is the trace of the stress tensor; $\boldsymbol{s}=\operatorname{dev}[\boldsymbol{\sigma}]=$ $\boldsymbol{\sigma}-\frac{\operatorname{tr}[\boldsymbol{\sigma}]}{3} \boldsymbol{I}$ is the deviatoric part of the stress; $J_{2}$ is the invariant $J_{2}(\boldsymbol{s})=\frac{1}{2} s \cdot \boldsymbol{s}, \sigma_{0}$ is the nominal yield stress, and $\alpha>0$ is a material constant.

Fig. 2.1 depicts Drucker-Prager's yield surface in the space of principal stresses $\left(\sigma_{1}, \sigma_{2}, \sigma_{3}\right)$ when the isotropic hardening variable is zero. This is a section of the yield surface in $\left(\sigma_{1}, \sigma_{2}, \sigma_{3}, q\right)$ space, which is not easy to visualize, and tries to show its non-smoothness. In this four dimensional space, $F=0$ is a hypersurface of dimension 3 and the non-smoothness is restricted to a curve at the intersection of the yield surface and the axis of hydrostatic stress, a curve defined as

$$
\mathcal{C}=\mathbb{E} \cap\{(\boldsymbol{\sigma}, q), \operatorname{tr}(\boldsymbol{\sigma})=0\},
$$

and whose projection onto principal stresses space is the apex of the cone in Fig. 2.1.

The inviscid solution of Drucker-Prager model requires explicitly calculate the projection $\Sigma^{t r} \mapsto \bar{\Sigma}^{t r}$ of a generalized stress onto the yield surface. For this particular case, the first thing to do is to identify whether the trial stress $\boldsymbol{\Sigma}^{t r}$ belongs to the normal cone to the smooth part of the yield surface or to the normal cone of the apex. Depending on this two cases, different projection formulas need to be employed. In both cases, the generalized stress $\bar{\Sigma}^{t r}$ is the unique, closest stress on the yield surface. Details of the implementation of such projection can be found, e.g., in Souza Neto, Peric, and Owen [42] and Loret and Prevost [54]. 


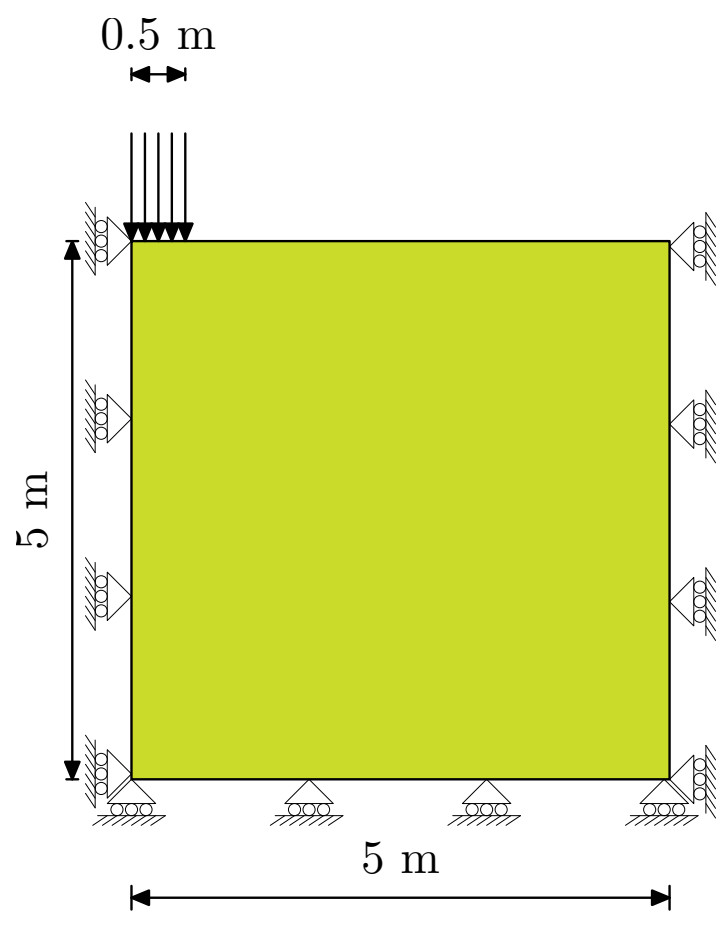

FIGURE 2.2: Strip footing geometry, boundary conditions, and loading.

\subsubsection{Numerical examples}

We show next the performance of several simulations where the integration algorithms developed previously are applied to the solution of boundary value problems. In all cases, we employ finite element discretizations with bi- or tri-linear elements and assumed strain (B-bar) formulation [55] to avoid volumetric locking. In the examples, we have employed kinetic potentials as defined in Eq. (2.25), with the function $J$ of the form

$$
J(x)=\frac{x^{n+1}}{n+1},
$$

and the parameter $\delta$ equal to the nominal yield stress $\sigma_{0}$. The convexity of these potentials and the result of Section 2.3.4 imply the pointwise non-negative dissipation the discrete solutions, which has been verified in all the examples.

\section{Strip footing}

In this example we address the classical benchmark that simulates the plastic collapse of a strip footing on a soil under plain strain conditions due to an applied load. The dimensions of the soil section are $10 \times 5 \mathrm{~m}^{2}$, but due to the symmetry of the geometry we only discretize half of the domain. See Fig. 2.2 for an illustration. In the simulation, hence, we consider a square shaped slice of the soil with an area of $5^{2} \mathrm{~m}^{2}$. On the left-top corner of the simulated domain, a downwards vertical displacement is applied, modeling the strip footing.

A $30 \times 30$ mesh of identical quads is employed first for the computations, which are carried under quasistatic conditions. Since yielding in soils is a pressure dependent phenomenon, we obtain a solution to the footing problem using viscoplastic models based on Drucker-Prager yield criterion without hardening. The constants of the model are given in Table 2.1. 


\begin{tabular}{|c|c|c|c|c|}
\hline$E$ & $\nu$ & $\alpha$ & $\tau$ & $\sigma_{0}$ \\
\hline $10 \mathrm{GPa}$ & 0.48 & 0.0016 & $0.5 \mathrm{~ms}$ & $848.7 \mathrm{kPa}$ \\
\hline
\end{tabular}

TABLE 2.1: Material parameters for viscoplastic Drucker-Prager model for strip footing test, section 2.4.3.

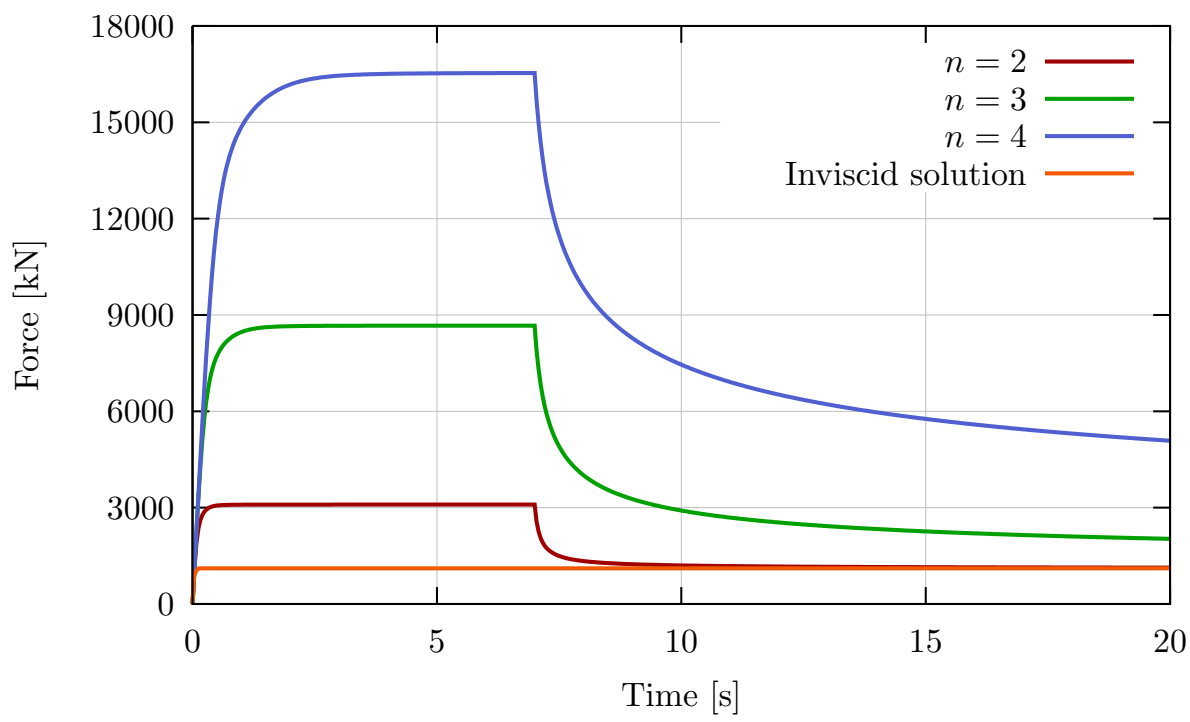

FIGURE 2.3: Forces on footing for different exponent values with Drucker-Prager plasticity criterion.

The simulation is initially driven with a controlled displacement of the footing at a constant velocity of $6.6 \mathrm{~mm} / \mathrm{s}$, during the first 7 seconds. After the loading phase, the position of the footing is held constant, allowing the soil to relax towards the inviscid (elastoplastic) solution. Fig. 2.3 shows the resultant forces measured on the footing during this process: after the loading, the resultant peaks, and then smoothly decreases as the soil relaxes. The problem is solved with three different potential laws for the kinetic function $J$, using exponents $n=2,3$, and 4 .

We repeat this simulation, now using a Tresca plasticity criteria with the data given in Table 2.2. to show explicitly that the viscoplastic solution converges to the inviscid one when the soil is allowed to relax for a long time, a solution that coincides with the bearing capacity of the footing as given by Prandtl and Hill [56]. This theoretical result shows that, for the limit load, the ratio between the pressure under the footing, $P$, and the cohesion parameter of the plasticity criteria $c$, is around 5.14. Fig. 2.4 depicts the ratio $P / c$ as a function of time for the footing benchmark and three different meshes of $30^{2}, 60^{2}$ and $80^{2}$ elements, respectively. The plots show that, as the mesh of the model is refined, the pressure approaches the reference solution. The time, $t_{L}$, in that figure is the loading time, $7 \mathrm{~s}$, used as normality factor of the simulation time.

\begin{tabular}{|c|c|c|c|c|}
\hline$E$ & $\nu$ & $\tau$ & $n$ & $\sigma_{0}$ \\
\hline $10 \mathrm{GPa}$ & 0.48 & $0.5 \mathrm{~ms}$ & 2 & $848.7 \mathrm{kPa}$ \\
\hline
\end{tabular}

TABLE 2.2: Material parameters for viscoplastic Tresca model in strip footing test, section 2.4 .3 .

Finally as we stated in Eq. 2.44, the convexity of the kinetic potential $\Xi$, ensures positive dissipation. As a check of this condition in Fig 2.5, we plot the dissipation 


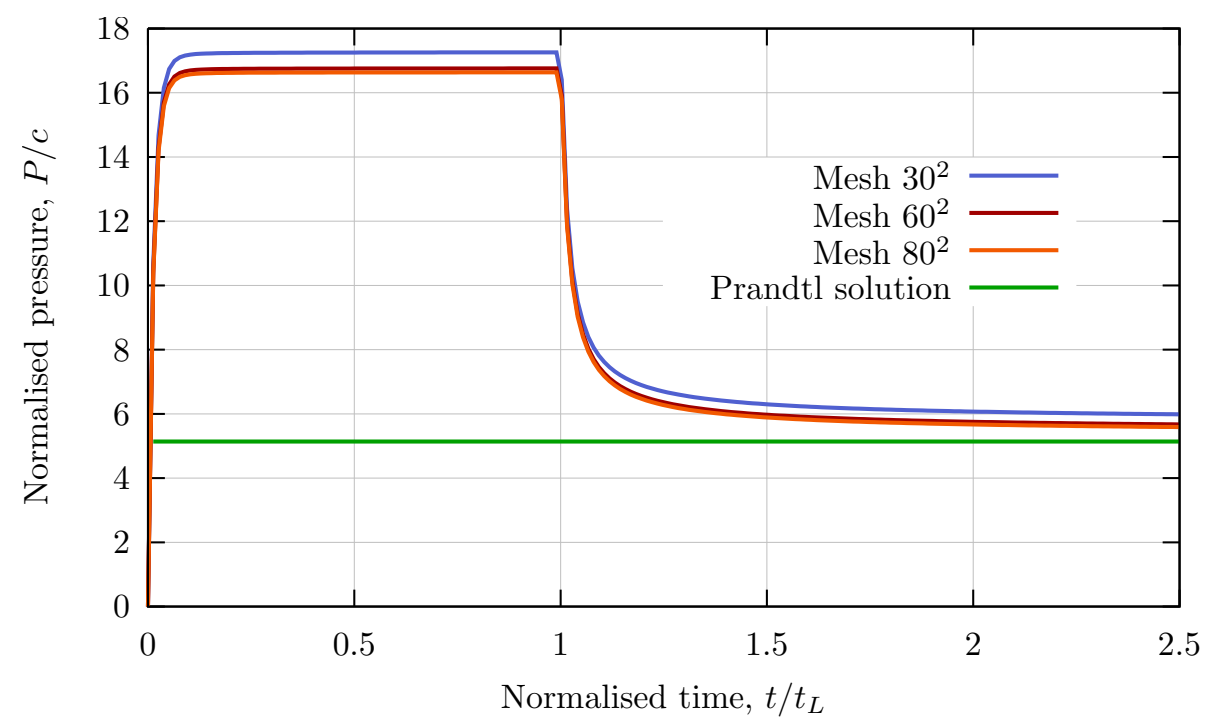

FIGURE 2.4: Strip footing simulation. Pressure to cohesion ratio as a function of time. Pressure in the strip footing and theoretical solution for Tresca plasticity criterion.

in the example performed with the material depicted in Table 2.2

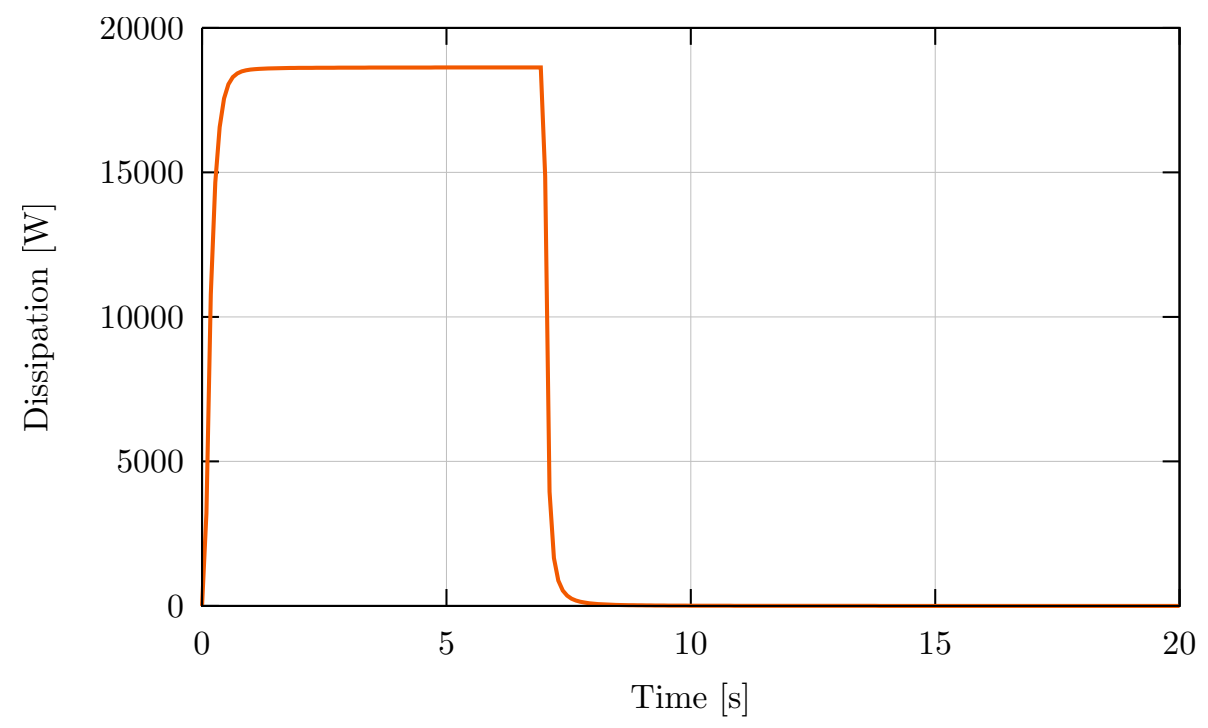

FIGURE 2.5: Dissipation of the strip footing problem performed with tresca plasticity model and mesh with $80^{2}$ elements. This dissipation is the volume integral over the body of the dissipated energy at each integration point.

\section{Traction test}

We simulate now a quasistatic tensile test on a cylindrical specimen of radius $10 \mathrm{~mm}$ and length $100 \mathrm{~mm}$, made of a material with a viscoplastic response based on Tresca's yield function. See Table 2.3 for the material properties. The numerical model consists of a finite element mesh of one eighth of the cylinder, employing 10 and 50 elements (hexahedrons) in the radial and axial directions, respectively.

In order to show the influence of the relaxation time and the dependence with the strain rate two series of simulations have been performed. In the first one, we vary 


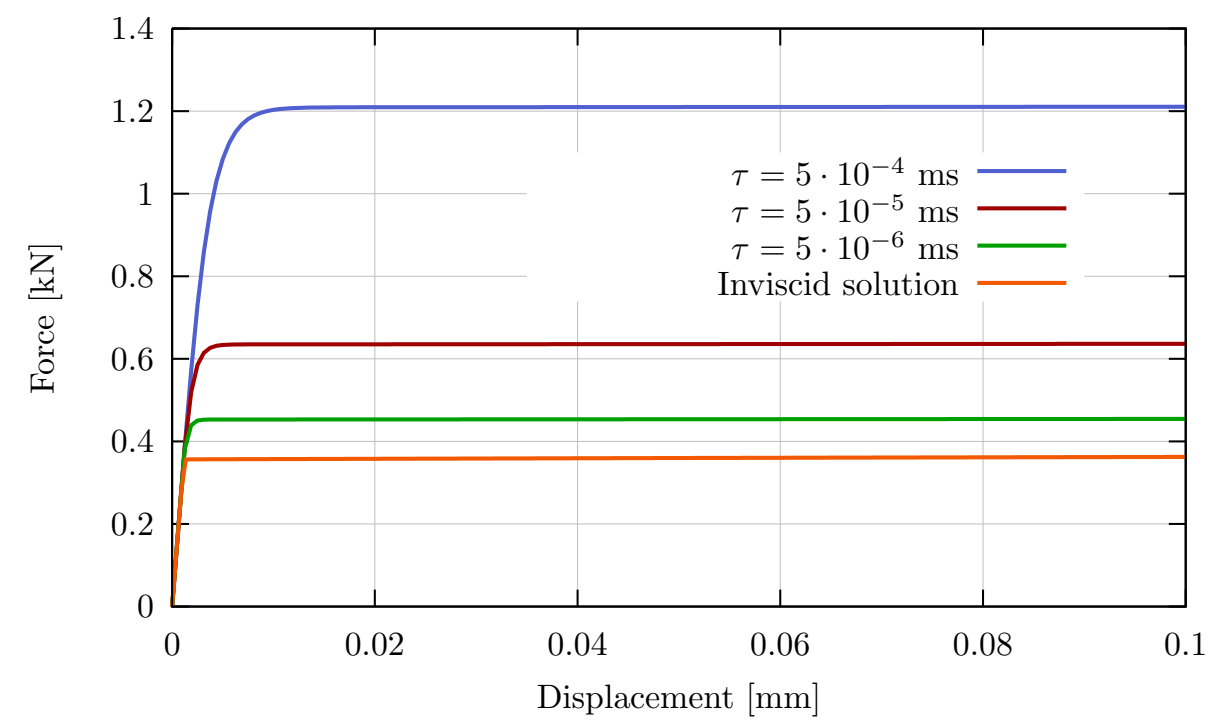

FIGURE 2.6: Forces for different values of relaxation time in cylinder traction test.

the relaxation time of the material, keeping all other material properties unchanged to see its effects on the response to a constant strain loading rate of $5 \mathrm{~s}^{-1}$. In Fig. 2.6 we observe that there is a direct relationship between the relaxation time, $\tau$, and the instant and value of the peak force.

In the second set of simulations, we study the sensibility of the original model to different loading strain rates. The solutions, depicted in Fig. 2.7 confirm that the material stiffens its response as we increase the stretching rate.

\begin{tabular}{|c|c|c|c|c|}
\hline$E$ & $\nu$ & $H_{\text {iso }}$ & $n$ & $\sigma_{0}$ \\
\hline $210 \mathrm{GPa}$ & 0.33 & $10 \mathrm{MPa}$ & 2 & $5 \mathrm{MPa}$ \\
\hline
\end{tabular}

TABLE 2.3: Material parameters for viscoplastic Tresca model in traction test, section 2.4 .3

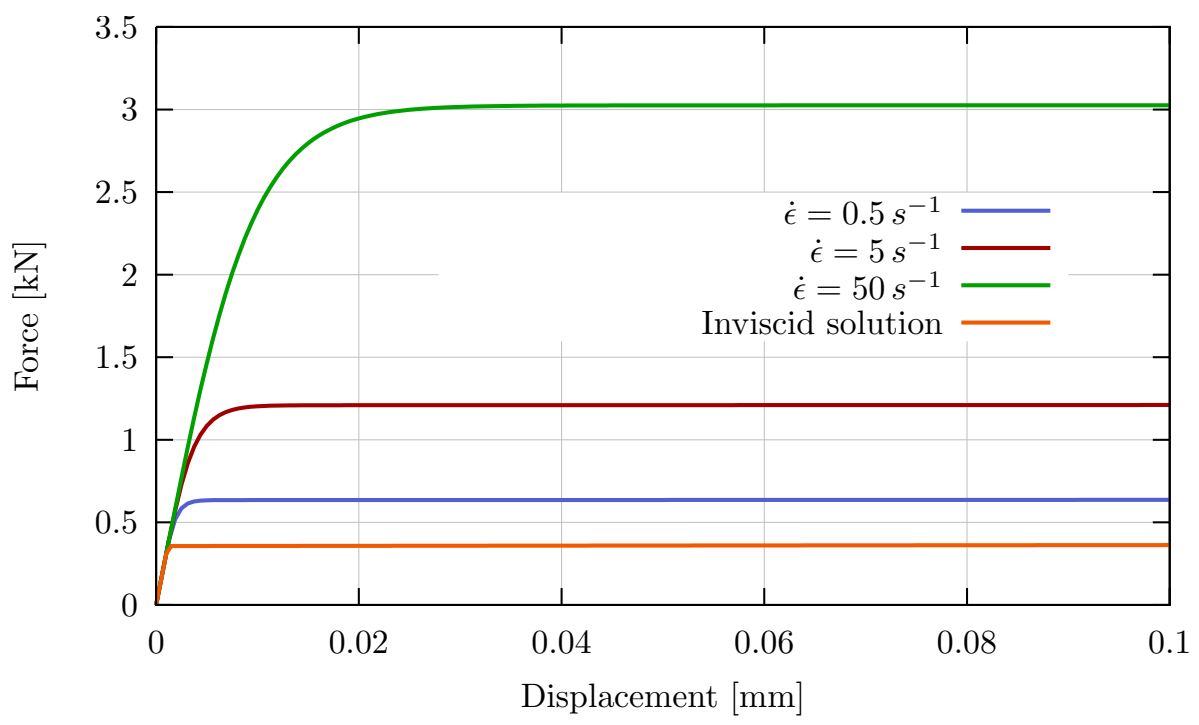

FIGURE 2.7: Forces for different strain rates in cylinder tensile test for $\tau=5 \cdot 10^{-5} \mathrm{~ms}$. 

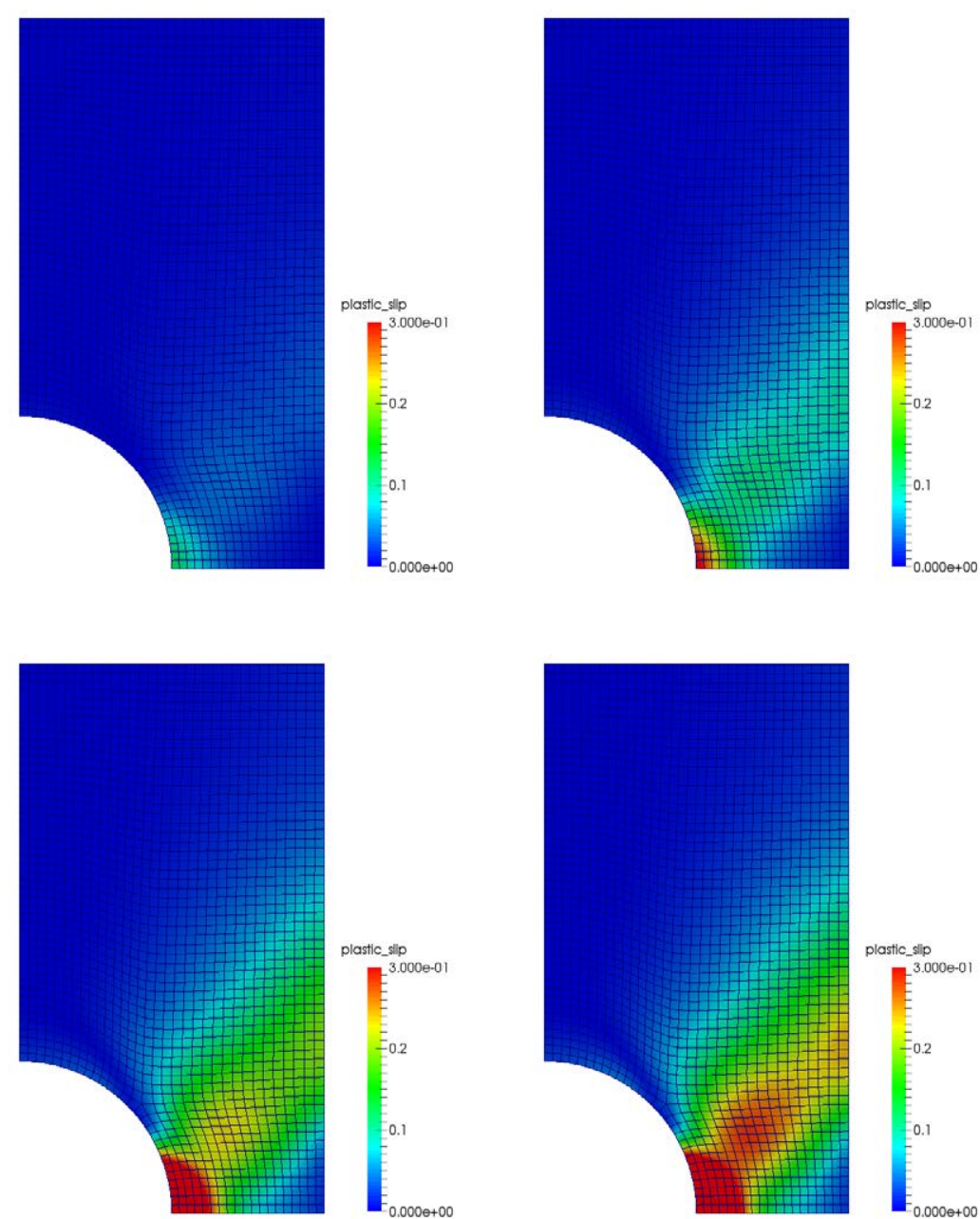

FIGURE 2.8: Effective plastic strain in perforated plate. From left to right, top to bottom, solutions at time $t=78,145,278$ and $331 \mathrm{~ms}$ for test with $n=5$.

\section{Perforated plate}

This example simulates a quasistatic tensile test, now on a viscoplastic perforated plate, considering only one quarter of its geometry (see Fig. 2.8) due to the symmetry. The plate has dimensions $20 \times 36 \times 1 \mathrm{~mm}^{3}$, the hole has radius equal to $5 \mathrm{~mm}$, and the material employed is based on a Tresca yield surface with parameters given in Table 2.4.

\begin{tabular}{|c|c|c|c|c|}
\hline$E$ & $\nu$ & $H_{\text {iso }}$ & $\tau$ & $\sigma_{0}$ \\
\hline $210 \mathrm{GPa}$ & 0.33 & $10 \mathrm{MPa}$ & $0.05 \mathrm{~ms}$ & $100 \mathrm{MPa}$ \\
\hline
\end{tabular}

TABLE 2.4: Material parameters for viscoplastic Tresca model in perforated plate, Sec. 2.4 .3 .

The plate is loaded by moving its top face at a constant velocity of $4 \mathrm{~mm} / \mathrm{s}$, and we consider several possible scenarios by varying the exponent in the viscoplastic kinetic law. The evolution of the loading forces in these tests is shown in Fig. 2.9. 


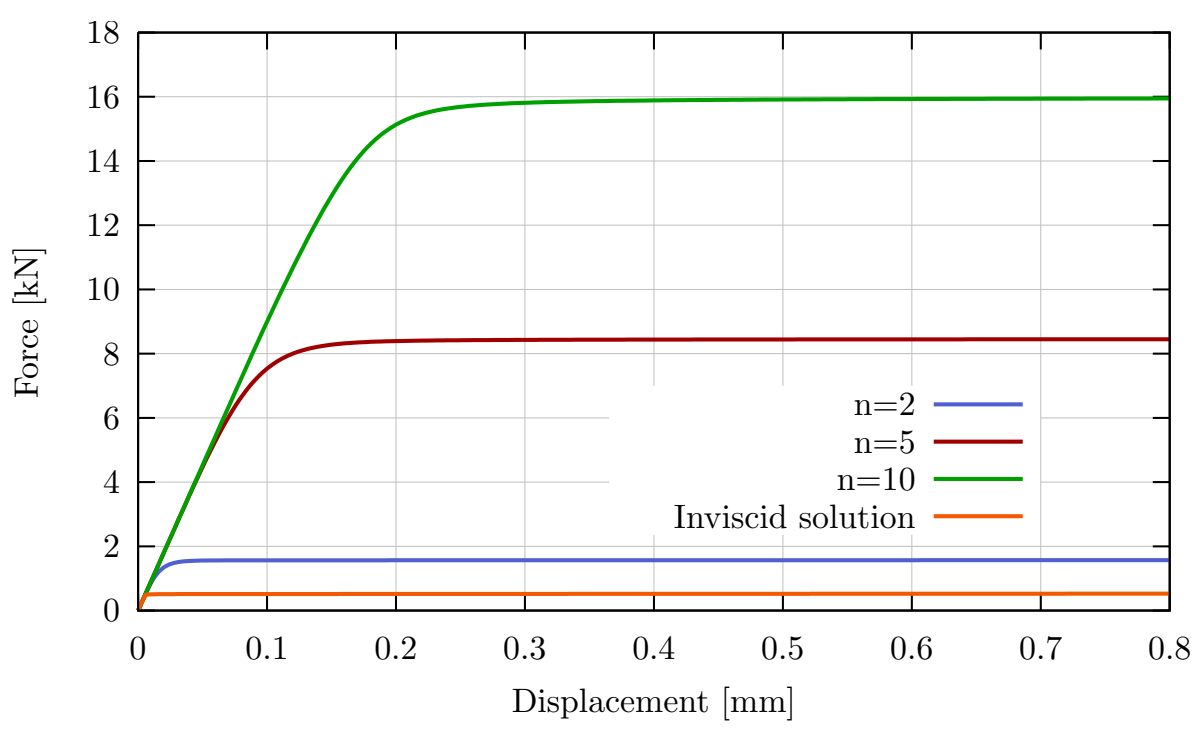

FIGURE 2.9: Forces for different values of exponent $n$ in the material of the perforated plate.

\section{Taylor bar}

In the last example we consider the Taylor bar benchmark to assess the constitutive model at high strain rates. The aim of this example is to show the viscoplastic behaviour at an order of magnitude in strain rate that is characteristic of high speed impact. In this experiment, a cylinder of height $32.4 \mathrm{~mm}$ and radius $3.2 \mathrm{~mm}$ is launched towards a rigid wall at a normal velocity of $280 \mathrm{~m} / \mathrm{s}$. The discretisation employed is based again on finite elements, using 32 and 4 hexahedral elements in the axis and radial directions, respectively. The normal displacement of the nodes on the wall is constrained to be always zero and, during the simulation, we compute the normal forces exerted by the wall onto the impacting cylinder. For this simulation we use an explicit integration scheme, a viscoplastic material model for the impacter based on Tresca's yield function with parameters given in Table 2.5. and we extend the models developed in Sec. 2.3 to the finite strain regime using the ideas of [57].

\begin{tabular}{|c|c|c|c|c|}
\hline$E$ & $\nu$ & $H_{\text {iso }}$ & $\tau$ & $\sigma_{0}$ \\
\hline $117 \mathrm{GPa}$ & 0.35 & $100 \mathrm{MPa}$ & $10 \mu \mathrm{s}$ & $400 \mathrm{MPa}$ \\
\hline
\end{tabular}

TABLE 2.5: Material parameters for viscoplastic Tresca model in Taylor bar, section 2.4 .3

Fig. 2.10 illustrates the sensibility of the reaction forces of the wall to different viscoplastic exponents in the kinetic function of the cylinder's material. While the inertia of the cylinder is being transmitted to the wall, forces show oscillations due to the multiple reflections of the shock waves. Finally in Fig. 2.11. we show the effective plastic strain of the bar during the impact for the specific case of a kinetic law with exponent $n=2$.

\subsection{Summary of main results}

This chapter presents a general class of integration algorithms for rate-dependent elasto-visco-plasticity. Their most significant features are that they are applicable for 


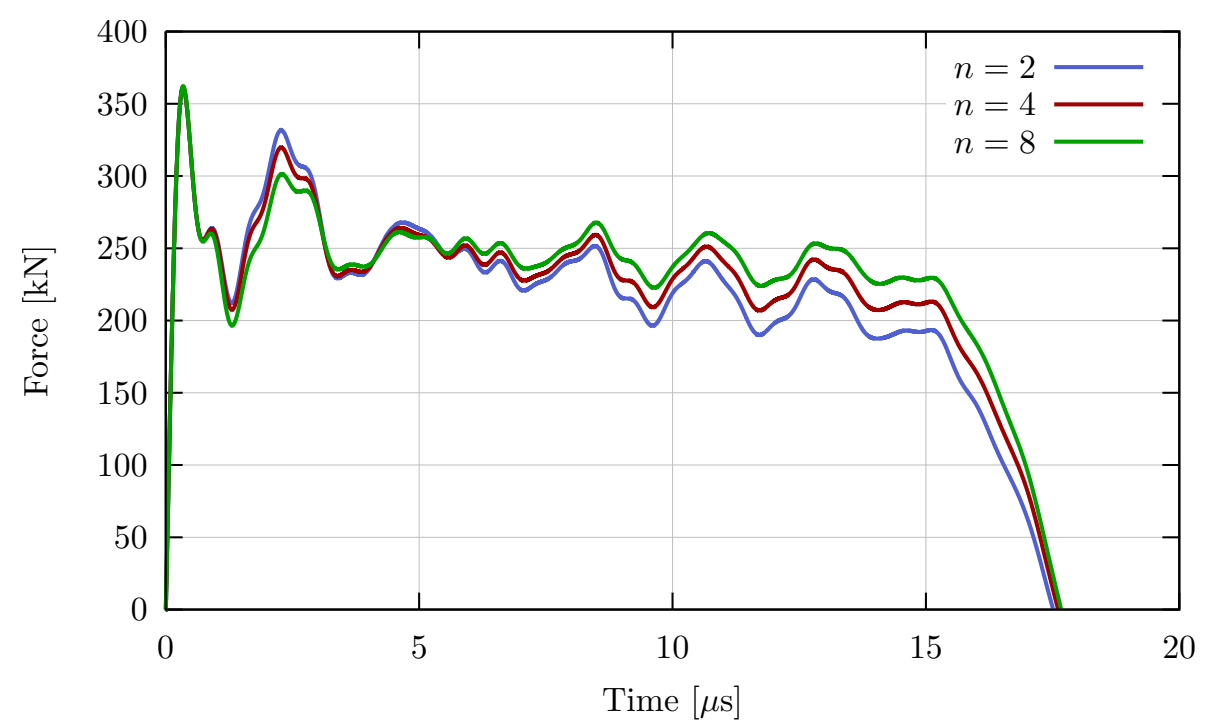

FIGURE 2.10: Forces for Taylor's bar impact

material models based on non-smooth yield functions, including hardening, and for fairly general viscoplastic flow rules.

The schemes shown in this chapter generalize existing ones in the literature, enriching their possible behaviors. Once the roles played by the rate-independent solution and the metric in generalized stress space are analyzed, we have been able to define very general expressions that can be used to model viscoplastic effects with great flexibility. Despite their generality, all the viscoplastic methods defined in this chapter, and for all smooth or non-smooth models, strictly satisfy a discrete dissipation inequality that mimics the behavior of the continuum models.

The methods that have been introduced can be used to integrate viscoplastic models based on smooth and non-smooth yield criteria, with or without isotropic and kinematic hardening. They accept a very simple structure that is very similar for all yield criteria, and based on geometric concepts such as distance and projection in the space of generalized stress. For these concepts to hold, the models are restricted to linear hardening, a limitation that can only be overcome at the expense of losing the simplicity of the solution. As particular examples, we presented the full details of the numerical schemes required to integrate viscoplastic models based on Tresca's and Druker-Prager's yield functions, with general (convex) kinetic potentials.

To conclude, all the ideas have been presented in the context of small strain kinematics. Nevertheless, once developed, they can be extended to the finite strain range using the ideas, for example, of [57].

\subsection{MUESLI}

The formulation for viscoplasticity presented along this chapter has been published [58] and also has been included and makes part of a larger compendium of constitutive models implemented in the open source library called MUESLI [59], the acronyme for Material UnivErSal LIbrary. This library is written in $\mathrm{C}++$ and encompasses a wide variety of materials with different formulations e.g. small strain kinematics, finite strain kinematics, fluids, rate dependent effects, damage, thermal effects, and so on. 

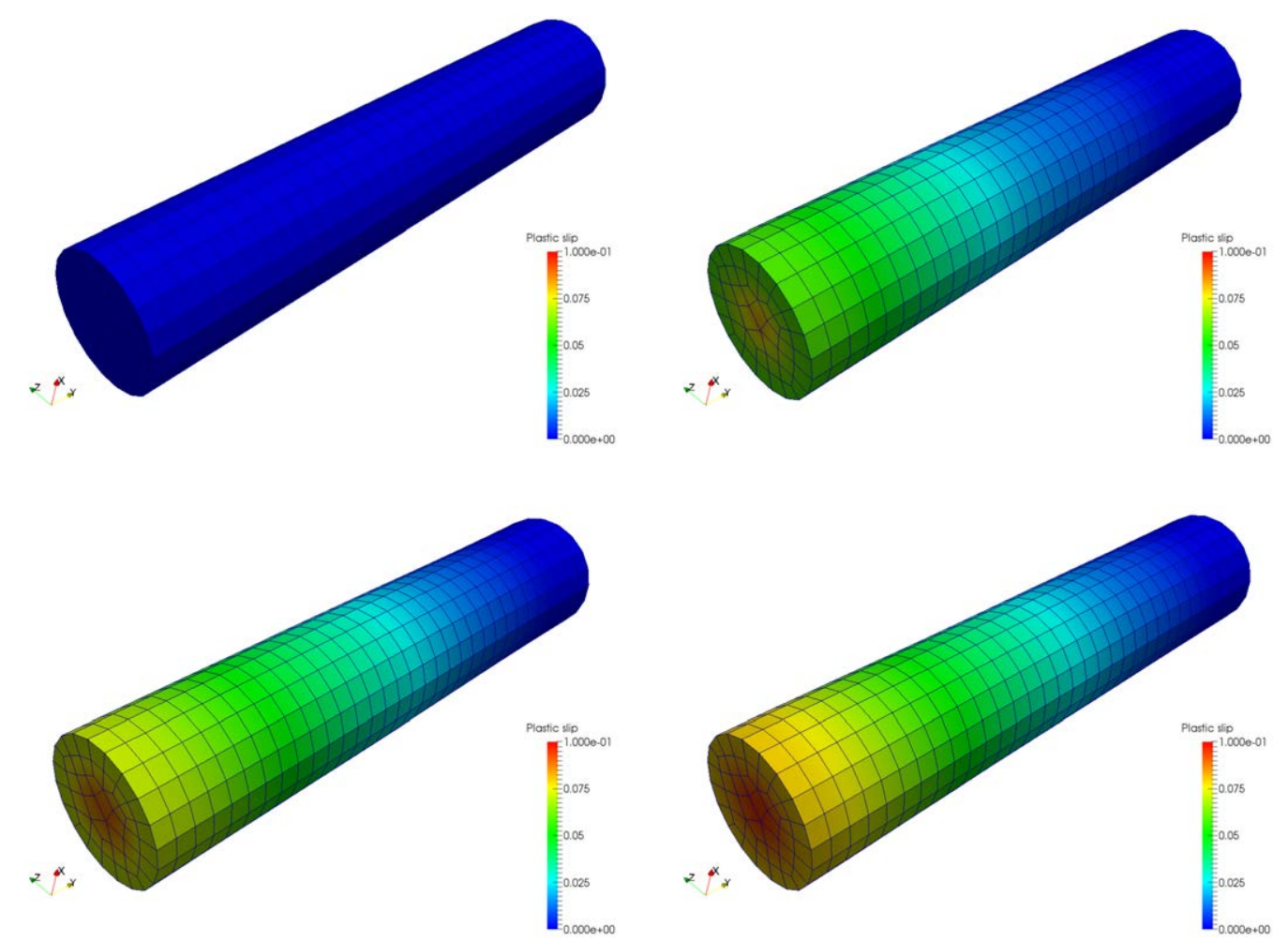

FIGURE 2.11: Effective plastic strain in Taylor's bar impact. From left to right, top to bottom, solutions at time $t=0,8,12,16 \mu \mathrm{s}$.

Material modelling is one of the cornerstones of Computational Mechanics. This body of knowledge has reached a mature state and many researchers have, at a certain point, implemented standard material models. This trend is reflected also in commercial codes and to such a degree that in most of them e.g. Abaqus, LS-DYNA, etc., there exists a section of the program called user subroutines where material models con be implemented. However, to the author's knowledge there is no attempt to provide a collection of constitutive models that is reliable, easy extendable and linkable to commercial codes. It is within this context that MUESLI has been developed. A comparison with MUESLI library implemented in the commercial code LS-DYNA is performed with an impact of a sphere with neohookean material. LS-DYNA uses first its own neohookean material and later it uses the equivalent material from the library MUESLI, as one can see the results obtained in von Mises stresses are almost equal, see 2.12. To conclude this section, the main features of MUESLI will be exposed justifying the requirements stated above.

\subsubsection{Reliable}

Muesli includes a set of tests that to a certain extent can verify the correctness of the formulations and might be very useful for debugging in complex models. These tests are based on hyperelastic formulations that assume the existence of an energy function from which the first and second derivatives give the stress and the stiffness tensor respectively. The tests numerically compute these derivatives and assert if the results are the same than in the implemented material subroutines. 

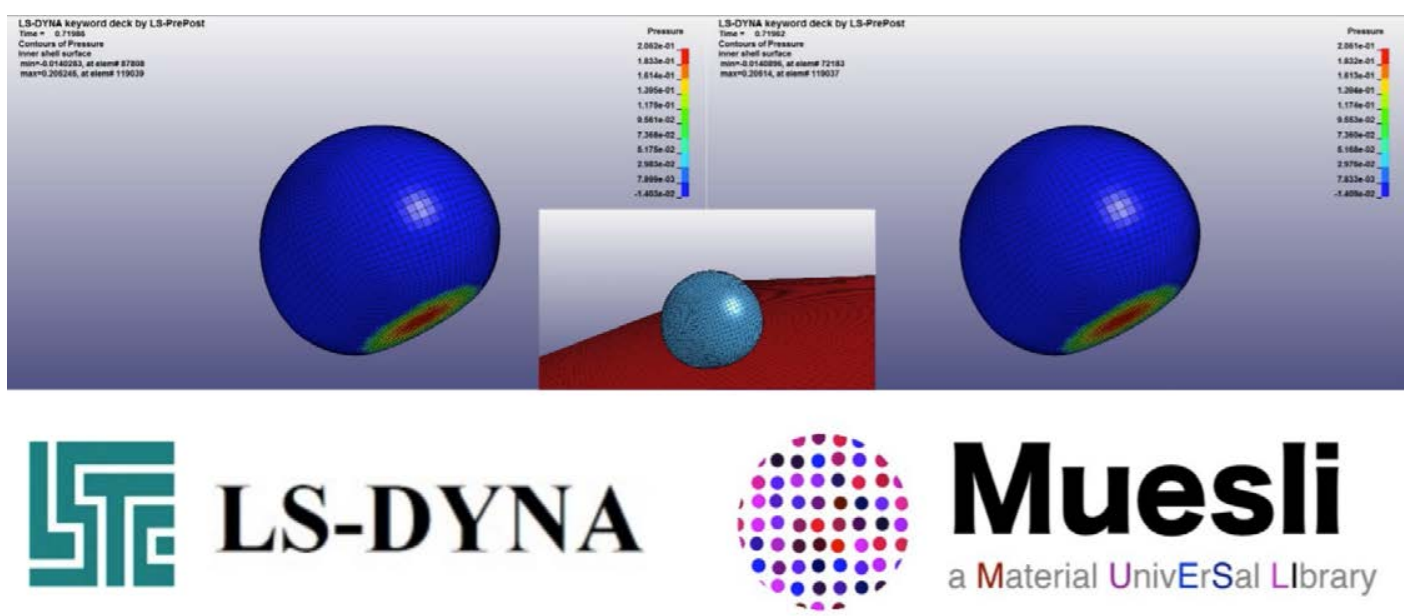

FIGURE 2.12: Neohookean sphere impacting onto a rigid plate, form left to right using LS-DYNA and MUESLI constitutive material respectively.

\subsubsection{Extendable}

From the fact that MUESLI is written in $\mathrm{C}++$ and the constitutive models have been structured in families and subfamilies, as a natural construction in object oriented languages, the inclusion of new material models becomes easy, being able to inherit features of the base material family. At the same time since languages such as $\mathrm{C}++$ possess operator overloading the implementation of material models can be done with ease because their mathematical description closely mimics the written code.

\subsubsection{Linkable}

The last feature of MUESLI that makes it appealing is that it can be employed with some comercial codes, in-built interfaces have been implemented allowing linkage with LS-DYNA and Abaqus. These interfaces take from the external code the data structure that is embedded in each material point and transfer it to MUESLI and finally the material point is updated in the external code. 



\section{Chapter 3}

\section{Meshless methods for high-speed impact}

The main characteristic of meshless method is that they are able to define the algebraic equations of the boundary value problem without using a mesh, and that it essentially relies on a set of nodes scattered within the domain to approximate the solution. The first publication in meshless methods dates back to the late seventies [34], [35] as a way to smooth continuous fields with a discrete system of particles as well as to obtain a continuous field of the variables associated to a set of discrete particle. Applied to astrophysics in its beginnings, it was applied to continuum mechanics afterwards. A great development since the nineties has been seen. Among them we can cite the diffuse element method (DEM) [60], see Fig. 3.1, as a natural extension from FEM substituting the shape function support restricted to the element boundaries with a local weighted least square approximation. Without extra hypothesis the DEM shape functions do not verify the delta Kronecker property. Later developments have been introduced as in [61], the differences with the later, are mainly the use of Lagrange multipliers to enforce boundary conditions and the use of more accurate derivatives. A cell structure which is independent of the nodes was used to integrate the weak form. Somehow equivalent methods are for example RKPM, [62], [63], that obtains kernel functions using correction functions and was also used as a correction for SPH kernel function [64].

As it seems evident from Fig 3.1 the sum of all the shape functions in their domain add up to one. This was first realised by [65] who established that one key characteristic of DEM is that the shape functions constitute a partition of unity. This will be generalised later with the partition of unity method (PUM) where the theoretical foundations of meshless methods as well as its convergence analysis was set [66], [67]. PUM relies on the existence of an open cover that contains, the body $\Omega$, see Fig. 3.2. and a set of shape functions verifying that their sum takes on the value one on each point of $\Omega$. Since in the previous method, the shape functions do not verify the Kronecker delta property, the imposition of essential boundary conditions becomes complex. However, over the next few years, some publications appeared, namely the natural element method (NEM) [68] and maximum entropy methods [69]-[71], with the PUM as cornerstone. The former is based on the Voronoi diagram associated with a set of nodes and its dual Delaunay tessellation. This generates a system of coordinates, shape functions, based on a Lebesgue measure and to a certain extent equivalent to the definition of barycentric coordinates. This endows the shape functions with nice properties such as the Kronecker delta property, nonnegativeness and as already mentioned due to its nature constitutes a partition of unity method. The latter relies on a convex optimisation for the maximisation of information obtained from the set of nodes using Jaynes' entropy [72] and the minimisation of a width defined through a certain measure to enforce locality of the shape 


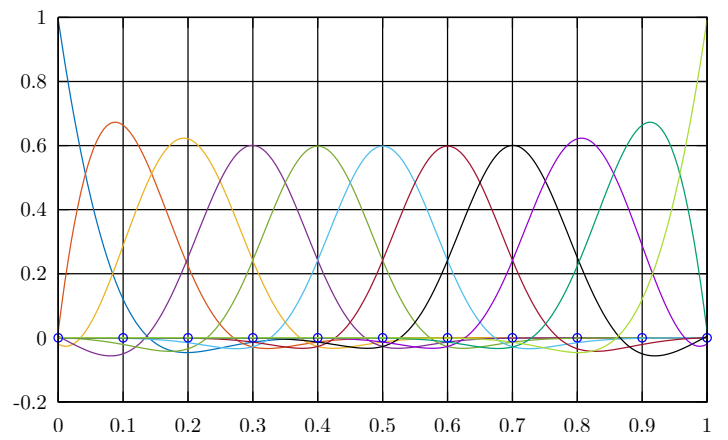

FIGURE 3.1: DEM quadratic shape functions in interval [0,1] for 11 nodes.

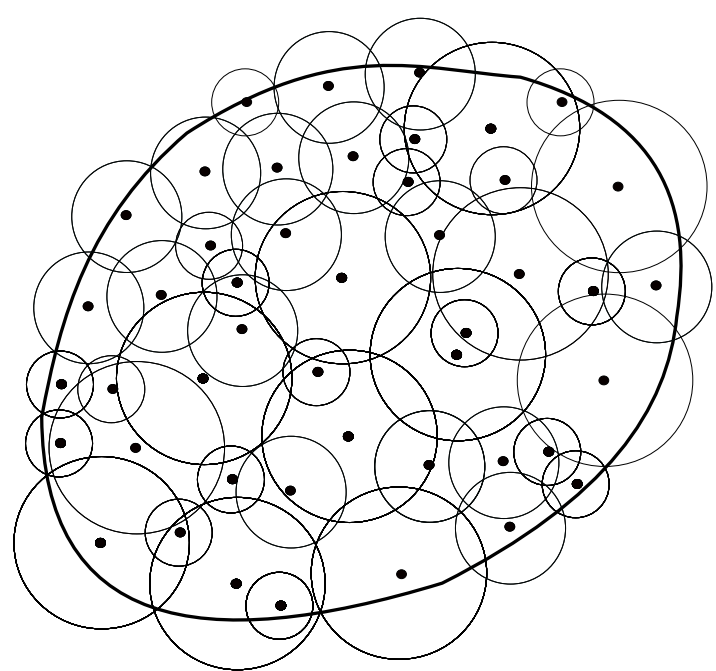

FIGURE 3.2: Covering in 2-D.

functions. Both methods have features in common, see Eq 3.7, that allow to include them in a more general group of methods called convex approximation schemes.

Another aspect of meshless method is the numerical integration. Most of them are based on the integration of the weak form using a background cell e.g. [61] or a nodal integration scheme, see [73]-[75]. However, some of them take directly the strong form and use it with a collocation approach, this makes the method very simple and fast, but suffer from instabilities [76], methods that uses this approach are e.g. SPH and radial collocation point method, see [64], [77]. Research on meshless methods has reached a mature state with extensive literature which among them we can cite the monographs [64], [76], [78], and detailed reviews of the available meshless methods [79]-[81].

In this work, the attention is mainly focused on two methods, SPH and Max-Ent addressed in Sec. 3.1 and Sec. 3.2, respectively. This choice is justified as two completely different meshless methods both with advantages and shortcomings. SPH is known for its easy implementation and reduced computational cost which for discretisations with thousands of particles this might be a key point. At the same time, it suffers from instabilities and lacks the mathematical framework to prove convergence. On the other hand Max-Ent might have the desired properties for any meshless method such as a weak Kronecker-delta property and the partition of unity of its shape functions, at the expense of being very costly computationally. 


\subsection{SPH method}

Developed in 1977 simultaneously by Lucy [34] and Gingold and Monaghan [35], in contrast with the particle discretisation methods that uses a set of points to discretise the continuum, the original application was used to obtain a continuum field from a discrete system. Some of these applications are astrophysics and quantum mechanics. However, in computational mechanics the SPH method has been adopted in the reverse way, using it to discretise the continuum.

\subsubsection{SPH interpolation}

SPH method is based on the notion of Dirac delta function that has the property

$$
F(\boldsymbol{x})=\int_{\mathbb{R}^{d}} \delta\left(\boldsymbol{x}^{\prime}-\boldsymbol{x}\right) F\left(\boldsymbol{x}^{\prime}\right) d \Omega_{\boldsymbol{x}^{\prime}}
$$

The Dirac delta function cannot be used numerically since it lacks properties such as continuity and differentiability. The SPH method tries to mimic this function by using a weight function $\Phi_{h}(\boldsymbol{x})$ and a set of nodes $I$ with coordinates $\boldsymbol{x}_{I}$ as a discretised version of the Dirac delta function that satisfies:

$$
F(\boldsymbol{x}) \approx \sum_{I} \Phi_{h}\left(\boldsymbol{x}-\boldsymbol{x}_{I}\right) F\left(\boldsymbol{x}_{I}\right) \Delta V_{I}
$$

Eq. (3.1) along with the property

$$
\int_{\mathbb{R}^{d}} \delta(\boldsymbol{x}) d \Omega_{\boldsymbol{x}}=1
$$

induce discrete counterparts that are the partition of unity and the Kronecker-delta property, and despite the fact that the weight functions do not verify the last property, they will require that

$$
\begin{aligned}
\int_{\mathbb{R}^{d}} \Phi_{h}(\boldsymbol{x}) d \boldsymbol{x} & =1, \\
\lim _{h \rightarrow 0} \Phi_{h}(\boldsymbol{x}) & =\delta(\boldsymbol{x}) .
\end{aligned}
$$

An extra feature of the weight functions is that they should be sufficiently smooth.

$$
\Phi_{h}(\boldsymbol{x}) \in C_{0}^{k}\left(\mathbb{R}^{d}\right), k \geq 1 .
$$

\subsubsection{SPH instabilities and artificial viscosity}

One of the well known shortcomings of SPH method is the so-called tension instability [82]. For a region in the discretised body under tensile stresses, any particle in that region when submitted to a perturbation will increase exponentially its velocity reaching a steady state with oscillating cluster of particles, ruining the homogeneity of the initial particle distribution. This instability is generated by the collocation method used to solve the equilibrium equations [76].

\subsection{Max-Ent}

In this chapter, we introduce the work done by [70], a meshless method that obtains the shape function through an optimisation scheme based on convex combinations. 
Therefore, the method inherits some nice results that arises directly from the nature of convex combination. Assuming that our domain $\Omega$ is represented by the convex hull of a set of $N$ nodes, $X=\left\{\boldsymbol{x}_{a}, a=1, \ldots, N\right\} \subset \mathbb{R}^{d}$ then its convex hull is expressed as

$$
\operatorname{conv} X=\left\{\boldsymbol{x} \in \mathbb{R}^{d} \mid \boldsymbol{x}=\boldsymbol{X} \boldsymbol{\lambda}, \boldsymbol{\lambda} \in \mathbb{R}_{+}^{N}, \mathbf{1} \cdot \boldsymbol{\lambda}=1\right\},
$$

where the matrix $\boldsymbol{X}$ has as columns the coordinates of the position of the points in $X$, with all the elements in $\boldsymbol{\lambda}$ greater or equal to zero. Therefore, if the values of $\boldsymbol{\lambda}$, associated with each of the nodes of $X$ and the position within the convex hull $\boldsymbol{x}$, are taken as shape functions $p_{a}(\boldsymbol{x})$, we will obtain the properties:

$$
\begin{gathered}
\sum_{a=1}^{N} p_{a}(\boldsymbol{x})=1 \quad \forall \boldsymbol{x} \in \operatorname{conv} X, \\
\sum_{a=1}^{N} p_{a}(\boldsymbol{x}) \boldsymbol{x}_{a}=\boldsymbol{x} \quad \forall \boldsymbol{x} \in \operatorname{conv} X,
\end{gathered}
$$

along with the non-negativity of $p_{a}$. In general, when $N>d+1$ the solution for $\lambda$ in Eq. 3.6 is not uniquely determined. The LME approximation scheme takes the convex combination that maximizes both the entropy, which is related with the least-biased solution, maximum influence of each node $\boldsymbol{x}_{a}$ and minimizes the width, seeking for locality in the solution. Therefore the LME problem becomes

$$
\begin{aligned}
& \text { For fixed } \boldsymbol{x} \text { minimize } \beta U(\boldsymbol{x}, \boldsymbol{p})-H(\boldsymbol{p}) \\
& \text { subjected to: } p_{a} \geq 0, a=1, \ldots, N \\
& \qquad \begin{array}{l}
\sum_{a=1}^{N} p_{a}(\boldsymbol{x})=1 \\
\sum_{a=1}^{N} p_{a}(\boldsymbol{x}) \boldsymbol{x}_{a}=\boldsymbol{x}
\end{array}
\end{aligned}
$$

which has a unique solution provided that $x \in \operatorname{conv} X$. In the problem statement Eq. 3.8, $\boldsymbol{p}$ is the vector with the shape functions $p_{a}$, the locality is obtained with $U(\boldsymbol{x}, \boldsymbol{p})$ that has the form:

$$
U(\boldsymbol{x}, \boldsymbol{p})=\sum_{a=1}^{N} p_{a} d^{2}\left(\boldsymbol{x}, \boldsymbol{x}_{a}\right),
$$

where $d(\cdot, \cdot)$ is a distance and the parameter $\beta \in(0,+\infty)$ tunes the steeper decay of shape functions. Finally, the least-biased information inferred from nodes is reached by the term $H(\boldsymbol{p})$ expressed as:

$$
H(\boldsymbol{p})=-\sum_{a=1}^{N} p_{a} \log p_{a} .
$$

\subsubsection{Weak Kronecker-delta property}

Assuming, as stated at the beginning of this chapter, that $\Omega=\operatorname{conv} X$, all convex approximations exhibit the so-called weak Kronecker-delta property which positions them in an advantageous situation compared with others meshfree approximations. This property means that for a convex approximation $\boldsymbol{p}:=\left\{p_{b}(\boldsymbol{x})\right\}$ in $\Omega$, given $F$ a 


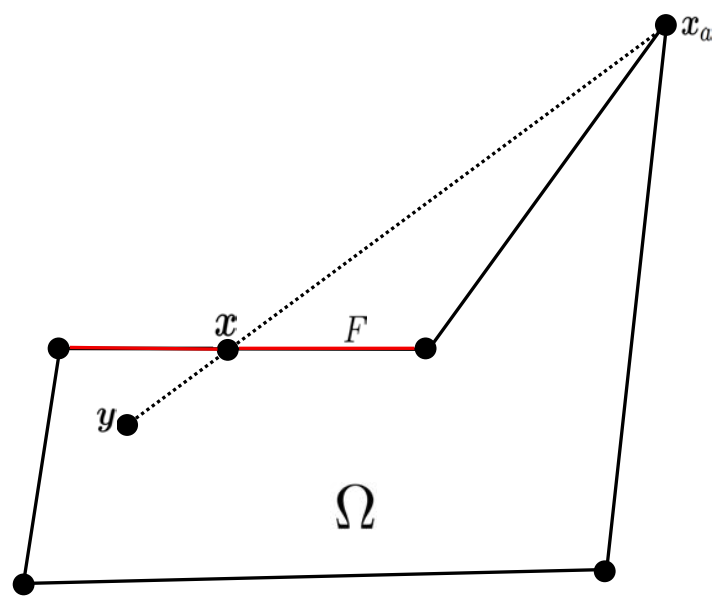

FIGURE 3.3: Non-convex domain.

face of $\Omega$, and $\boldsymbol{x}_{a} \notin F$, then $p_{a}=0$ on $F$. As shown in [70], the fact that there is a closed line contained in $\Omega$ that also contains $x \in F$, such that

$$
\boldsymbol{x}=\sum_{b} p_{b}(\boldsymbol{x}) \boldsymbol{x}_{b}
$$

where the closed line is defined as

$$
t \in[0,1] \rightarrow \boldsymbol{y} t+(1-t) \boldsymbol{x}_{a}
$$

with

$$
\boldsymbol{y}=\frac{1}{\sum_{b \neq a} p_{b}(\boldsymbol{x})} \sum_{b \neq a} p_{b}(\boldsymbol{x}) \boldsymbol{x}_{b}
$$

implies $\boldsymbol{x}_{a} \in F$, thus weak Kronecker-delta property has to be verified. However, when the domain $\Omega$ is not convex, there might exist a point $x$ in the interior of the closed line such that the line itself is not contained in $\Omega$, see Fig. 3.3. In other words, $\boldsymbol{x}_{a} \notin F$ and it might be that $p_{a} \neq 0$ on $F$.

\subsubsection{Log-distance}

Another variations of Eq. 3.9 are to consider $U(\cdot, \cdot)$ not depending quadratically on the distance having as result other decay behaviours. Even distances based on the logarithm function could be used, redefining $U(\boldsymbol{x}, \boldsymbol{p})$ as

$$
\sum_{a=1}^{N} p_{a} \log \left(\frac{\left|\boldsymbol{x}-\boldsymbol{x}_{a}\right|}{h_{a}}\right) .
$$

$h_{a}$ is the parameter that gives fraction dimensionless, avoiding at the same time numerical issues with the formulation. In this work this parameter represents the least inter-nodal distance seen by $\boldsymbol{x}_{a}$. Using this distance, the parameter $\beta$ has to be dimensionless, therefore we refer to it as $\gamma$. The solution to the convex optimisation in Eq. 3.8 as shown in [70] with the logarithmic measure in Eq. 3.14 gives an expression for the shape functions as written below

$$
p_{a}(\boldsymbol{x})=\frac{1}{Z\left(\boldsymbol{x}, \boldsymbol{\lambda}^{*}\right)} \exp \left[-\gamma \log \left(\frac{\left|\boldsymbol{x}-\boldsymbol{x}_{a}\right|}{h_{a}}\right)+\boldsymbol{\lambda}^{*} \cdot\left(\boldsymbol{x}-\boldsymbol{x}_{a}\right)\right],
$$


where $Z\left(\boldsymbol{x}, \boldsymbol{\lambda}^{*}\right)$ is

$$
\sum_{a=1}^{N} \exp \left[-\gamma \log \left(\frac{\left|\boldsymbol{x}-\boldsymbol{x}_{a}\right|}{h_{a}}\right)+\boldsymbol{\lambda}^{*} \cdot\left(\boldsymbol{x}-\boldsymbol{x}_{a}\right)\right],
$$

and $\boldsymbol{\lambda}^{*} \in \mathbb{R}^{d}$ is

$$
\boldsymbol{\lambda}^{*}=\arg \min _{\lambda \in \mathbb{R}^{d}} \log Z(\boldsymbol{x}, \boldsymbol{\lambda}) .
$$

As explained in $[70]$, this locality measure yields interpolation functions that verify the Kronecker-delta property. This can be easily verified by noticing that the function $Z\left(\boldsymbol{x}, \boldsymbol{\lambda}^{*}\right)$, when $\boldsymbol{x}$ tends to one of the nodes $\boldsymbol{x}_{a}$, is infinite

$$
\lim _{\boldsymbol{x} \rightarrow \boldsymbol{x}_{a}} Z\left(\boldsymbol{x}, \boldsymbol{\lambda}^{*}\right)=\lim _{\boldsymbol{x} \rightarrow \boldsymbol{x}_{a}} \exp \left[-\gamma \log \left(\frac{\left|\boldsymbol{x}-\boldsymbol{x}_{a}\right|}{h_{a}}\right)+\boldsymbol{\lambda}^{*} \cdot\left(\boldsymbol{x}-\boldsymbol{x}_{a}\right)\right]=\infty,
$$

since all the terms are bounded above in $Z\left(\boldsymbol{x}, \lambda^{*}\right)$ except the term that is common in numerator and denominator in Eq. 3.15, it follows

$$
\lim _{\boldsymbol{x} \rightarrow \boldsymbol{x}_{a}} p_{a}(\boldsymbol{x})=1,
$$

and

$$
\lim _{\boldsymbol{x} \rightarrow \boldsymbol{x}_{b}} p_{a}(\boldsymbol{x})=0,
$$

and by continuity we define

$$
p_{a}\left(\boldsymbol{x}_{b}\right)=0, \quad p_{a}\left(\boldsymbol{x}_{a}\right)=1 .
$$

\subsubsection{Remeshing}

The solution to the convex optimisation Eq. 3.8) is equivalent to the solution of Eq. (3.17) which can be done through a Newton-Raphson method and in some cases it might be useful to use a line search algorithm to improve convergency. However, solving problem (3.17) is very demanding from the computational viewpoint since the problem has to be solved for each of the Gauss points. Nevertheless, due to the rapid decay of their support one can define a radius $r$ and the ball $B_{r}(\boldsymbol{x})=\{\boldsymbol{y} \in$ conv $X:|\boldsymbol{y}-\boldsymbol{x}|<r\}$ centered at each Gauss point $\boldsymbol{x}$, from which the contribution of nodes $\boldsymbol{x}_{a}$ that are outside this ball is negligible, see Fig. 3.4 , therefore reducing the computational cost. In the phenomena of impact, soft bodies discretised with meshless methods might be sujected to large distortion. The discretising nodes $\boldsymbol{x}_{a}$ during this distortion could leave the domain $B_{r}(\boldsymbol{x})$ and others could enter the domain. Therefore, a reassignment of nodes $\boldsymbol{x}_{a}$ within $B_{r}(\boldsymbol{x})$ has to be done which implies recomputing the shape functions. Thus, for great distortions remeshing is necessary, wich is done by means of nearest neighbours algorithm [83].

In the paragraphs below the process of remeshing is explained. Before starting, let us define two sets, the set of all Gauss points $\mathcal{G}$ and the set of all nodes $\mathcal{N}$. Let us define the set $\mathcal{B}(\boldsymbol{x}) \subset \mathcal{N}$ as the nodes that are within $B_{r}(\boldsymbol{x})$ and finally the map that associates each $\boldsymbol{x} \in \mathcal{G}$ with $\mathcal{B}(\boldsymbol{x})$ is defined as $\mathcal{M}$. Thus, as summarised in Algorithm 1, at the beginning the reference coordinates of the Gauss points (coordinates in the initial configuration) are set to its current coordinates and the value of the gradient tensor $\boldsymbol{F}$ on each point is stored. Then the remeshing procedure uses a query algorithm that looks for neighbours within the $B_{r}(\boldsymbol{x})$ of each Gauss point $\boldsymbol{x}$, as result $\mathcal{M}$ is returned. Now, the shape functions can be computed for each set 


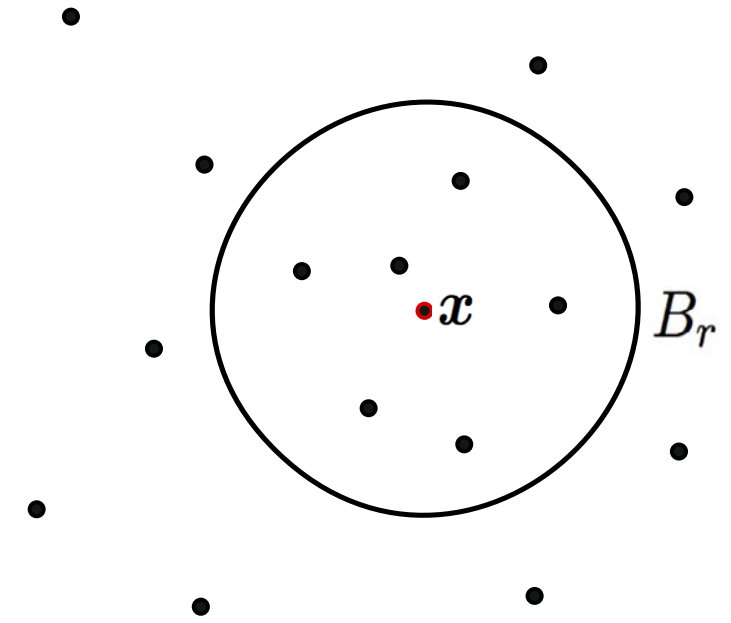

FIGURE 3.4: Ball for node search.

of nodes $\mathcal{B}(\boldsymbol{x})$. Finally the reference positions of the nodes $\boldsymbol{x}_{a}$ are set to the current coordinates and the displacement is set to zero.

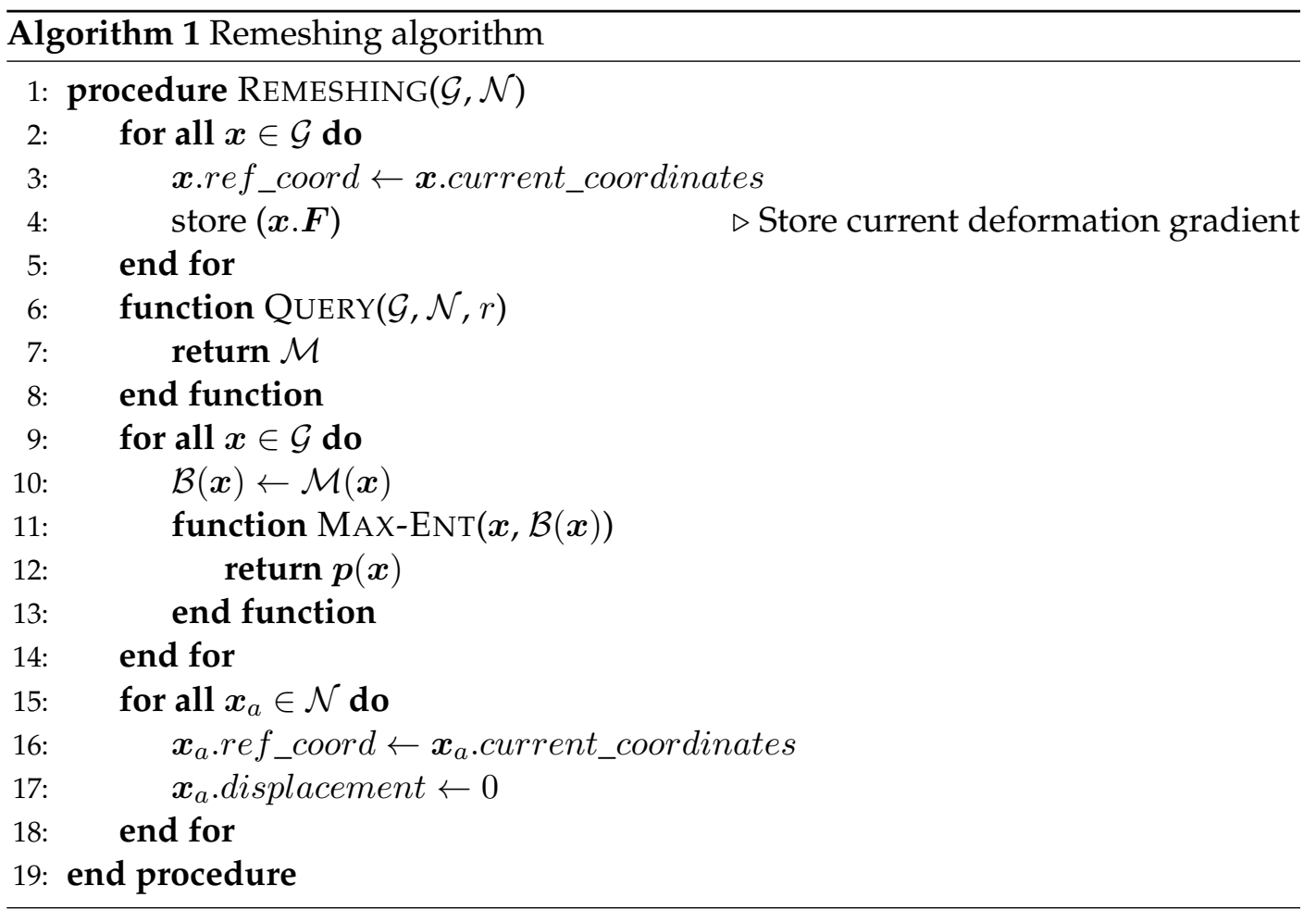

\subsection{Meshless Analysis of High-Speed Impact/Contact Prob- lem}

High speed impact often involves a large distortion of the bodies meeting in the event. For these cases FEM suffers from inaccuracies due to the distortion of the mesh. It is within this kind of applications that meshless methods show their great potential, since their numerical accuracy does not rely on a mesh that might be subjected to distortion. Meshless methods involved in contact can be either through interactions meshless-meshless bodies or meshless-FEM bodies. For the former, some 
ideas can be found in the optimal transportion meshfree method [40] which has common features with other material-point methods [84]. In these methods contact is accounted for through updates of the local neighbourhoods of material points, see Algorithm 1, that track the relative motion of colliding nodes from different bodies, leading to a cancelation of linear momentum wich ensures impenetrability.

Elapsed times in high speed impact are short, at most in the order of milliseconds. In such case, it is advisable to use explicit integration schemes, which are a standard choice. Now, let us briefly introduce the problem for $N$ deformable bodies, with no interaction among them through contact, referred to as $\mathcal{B}_{i}$ with $i=1, \ldots, N$ and $\mathcal{B}_{i}$ corresponding to an open subset of $\mathbb{R}^{3}$ with boundary $\partial \mathcal{B}_{i}$. Points on any of the bodies are denoted as $\boldsymbol{X}$ and we assume the existence of $N$ deformations $\varphi_{i}: \mathcal{B}_{i} \times[0, T] \rightarrow \mathbb{R}^{3}$. In addition, the boundary of each body $\partial \mathcal{B}_{i}$ is partitioned into disjoint subsets $\Gamma_{i}^{t}, \Gamma_{i}^{\varphi}$ referring to the boundary with Neumann conditions and Dirichlet conditions, respectively which satisfy

$$
\partial \mathcal{B}_{i}=\overline{\Gamma_{i}^{t} \cup \Gamma_{i}^{\varphi}} .
$$

The key classification of the contacting bodies is thus between those that possess a mesh and those that not, and we refer to these sets, respectively, as $\mathcal{F}$ and $\mathcal{M}$. We briefly describe the key features of the two types of spatial discretizations considered in the current work:

- Finite element discretisation: A body $\mathcal{B}_{i} \in \mathcal{F}$ is discretized with finite elements by means of a node set $\mathcal{N}_{i}$ and a triangulation $\mathcal{T}_{i}$ of tetrahedra or hexahedra. This triangulation partitions the domain into disjoint elements $e_{i}^{\alpha} \subset \mathcal{B}_{i}$ with vertices $n_{i}^{\alpha} \in \mathcal{N}_{i}$ and allows to construct piecewise polynomial functions $N_{i, \alpha}$ with the property $N_{i, \alpha}\left(n_{i}^{\gamma}\right)=\delta_{\alpha \gamma}$, where $\delta_{\alpha \gamma}$ refers to the Kronecker delta. The support of the function $N_{i, \alpha}$ is the set $\cup_{e_{i}^{\beta} \ni n_{i}^{\alpha}} e_{i}^{\beta}$.

- Meshfree discretization: Let $\mathcal{B}_{i} \in \mathcal{M}$, and define a finite node set $\mathcal{N}_{i}=\left\{n_{i}^{\alpha}\right\}$ on this domain. By definition, there might be a mesh defined on $\mathcal{B}_{i}$ or not, but we will never make use of it. The only condition on the discretization of this body is that there exists a collection of approximation functions $N_{i, \alpha}: \mathcal{B}_{i} \rightarrow \mathbb{R}$ such that linear combinations of the type (3.25) can be employed to approximate the deformation and velocity of the body. In what follows, we refer to bodies in $\mathcal{M}$ as meshfree bodies to indicate that there is no need for a mesh to be defined on them, although they might have it. There is much freedom in the choice of these functions. As stated before, they might be finite element shape functions if the body has a mesh, but can also be any meshfree function including those employed in the element free Galerkin method, the natural neighbor method, or any other of this sort (see, e.g. [64], for a review). In particular, and without any loss of generality, the simulations of Section 3.4.8 will make use of local maximum entropy approximation functions [70].

The nature of high-speed impact is governed by dynamic large-strain elastic plastic problems [76], within this context we can write the linear momentum conservation in its strong form, as follows:

$$
\begin{aligned}
\operatorname{Div} \boldsymbol{P}+\rho_{0} \boldsymbol{B} & =\rho_{0} \ddot{\boldsymbol{\varphi}}_{i} & & \text { in } \mathcal{B}_{i} \\
\boldsymbol{\varphi}_{i} & =\overline{\boldsymbol{\varphi}}_{i} & & \text { on } \Gamma_{i}^{\varphi} \\
\boldsymbol{P} \boldsymbol{N}_{i} & =\overline{\boldsymbol{T}}_{i} & & \text { on } \Gamma_{i}^{t}
\end{aligned}
$$


where Div is the material divergence, $\boldsymbol{P}$ the first Piola-Kirchhoff stress tensor, $\rho_{0}$ the reference density, $\boldsymbol{B}$ the volumetric force, $\bar{\varphi}_{i}$ a surface field of known deformations, $\overline{\boldsymbol{T}}_{i}$ the known tractions. Therefore, the Galerkin method applied to the conservation of linear momentum for a given body $\mathcal{B}_{i}$ can be written as

$$
\int_{\mathcal{B}_{i}} \rho_{0} \ddot{\boldsymbol{\varphi}}_{i}^{h} \cdot \boldsymbol{\eta}^{h} \mathrm{~d} V+\int_{\mathcal{B}_{i}} \boldsymbol{P} \cdot \operatorname{Grad} \boldsymbol{\eta}^{h} \mathrm{~d} V=\int_{\mathcal{B}_{i}} \rho_{0} \boldsymbol{B} \cdot \boldsymbol{\eta}^{h} \mathrm{~d} V+\int_{\Gamma_{i}^{t}} \overline{\boldsymbol{T}}_{i} \cdot \boldsymbol{\eta}^{h} \mathrm{~d} A,
$$

where $\boldsymbol{\eta}^{h}: \mathcal{B}_{i} \rightarrow \mathbb{R}^{3}$ is an arbitrary weighting function in the finite or meshfree space. Selecting the test function $\boldsymbol{\eta}^{h}$ to be of the form $\boldsymbol{\eta}^{h}=N_{i, \alpha} \boldsymbol{c}$, with $n_{i}^{\alpha} \in \mathcal{N}_{i}$ and $\boldsymbol{c} \in \mathbb{R}^{3}$, where the scalar functions $N_{i, \alpha}: \mathcal{B}_{i} \rightarrow \mathbb{R}$ are the standard finite element shape functions, if the body is discretized with a mesh, or some meshfree interpolation function if not. Finally assuming a discretisation of the deformation field $\varphi_{i}$ as follows

$$
f(\boldsymbol{X}) \approx f^{h}(\boldsymbol{X})=\sum_{\alpha} N_{i, \alpha}(\boldsymbol{X}) c_{i, \alpha},
$$

the equations of motion in matrix form follow

$$
\begin{aligned}
\boldsymbol{M}^{i} \ddot{\boldsymbol{\Phi}}^{i}+\boldsymbol{f}_{\text {int }}^{i} & =\boldsymbol{f}_{\text {ext }}^{i}, \\
\boldsymbol{f}_{\text {int }}^{i, \alpha} & =\int_{\mathcal{B}_{i}} \boldsymbol{P} \operatorname{Grad} N_{i, \alpha} \mathrm{d} V, \\
\boldsymbol{f}_{\text {ext }}^{i, \alpha} & =\int_{\mathcal{B}_{i}} \rho_{0} \boldsymbol{B} N_{i, \alpha} \mathrm{d} V+\int_{\Gamma_{i}^{t}} \overline{\boldsymbol{T}}_{i} N_{i, \alpha} \mathrm{d} A
\end{aligned}
$$

where $\boldsymbol{\Phi}^{i}, \boldsymbol{f}_{i n t}^{i}, \boldsymbol{f}_{\text {ext }}^{i}$ are the vectors collecting, respectively, the nodal deformations, internal forces, and external forces. The matrix $\boldsymbol{M}^{i}$ is the mass matrix of body $\mathcal{B}_{i}$ which, in order to later obtain an explicit method, is lumped into diagonal form. In other words, the $(i, j)$ component in the block relating nodes $\alpha$ and $\beta$ is

$$
M_{\alpha \beta}^{i j}=m^{i, \alpha} \delta_{\alpha \beta} \delta_{i j}
$$

with $m^{i, \alpha}$ the mass of node $n_{i}^{\alpha}$. We want to stress that in formulations that consider contact, an extra term referring to contact interaction between the $N$ bodies should be added to Eq. 3.24, using penalty method, Lagrange multipliers or other methods [85]. The former will lead to a formulation where only an extra term has to be added to Eq. (3.26) that accounts for carefully selected penalty forces whose magnitude, as a function of the penetration, is artificially selected [86]-[88]. Lagrange multipliers can be used to exactly verify the impenetrability constraints but this precludes keeping the explicit character in Eq. (3.26), see [87], [89].

There is a third approach that consists of two steps, first performing an unconstraint predictor step using Eq. (3.26) and second enforce the impenetrability constraints in a corrector step [87], [90], [91]. Thus, this predictor-corrector schemes are amenable to explicit integration. Within this family of algorithm we can also mention [92] that instead of using Lagrange multipliers to obtain the forces that ensure impenetrability, modifies separately positions and velocities to ensure impenetrability and perform the contact interaction. This approach can be understood as replacing a force by the effects it produces, change in velocity and position. Within this context, we present in the next section the contact algorithm that uses a predictorcorrector approach for contact based on velocity and position modifications of the interacting nodes. First we integrate the equations of motion using the central difference method, ignoring all possible contacts; then, we correct the positions and 
velocities to account for the pairwise interactions. To define the integration, we select $M+1$ instants $t_{n}=n \Delta t$, with $n=0,1, \ldots M$, where $\Delta t=T / M$ is the time step size, assumed for simplicity to be constant. Then, we define the approximations to the deformations, material velocities, and material acceleration, respectively, at each node and time $t_{n}$ to be $\varphi_{n}^{i, \alpha}, \boldsymbol{v}_{n}^{i, \alpha}, \boldsymbol{a}_{n}^{i, \alpha}$, where the index $i$ runs through all body labels and $\alpha$ through the node set in each body. Following the standard notation, we will refer to $f_{n+\gamma}$ as the convex combination $(1-\gamma) f_{n}+\gamma f_{n+1}$ for any nodal variable $f$, and $\gamma \in[0,1]$. Collecting the nodal deformations, into a global vector $\boldsymbol{\Phi}_{n}$, integration is performed as follows:

1) Calculate the predictor state

$$
\begin{aligned}
\tilde{\boldsymbol{a}}^{i, \alpha} & =\left(m^{i, \alpha}\right)^{-1}\left(\tilde{\boldsymbol{f}}_{\text {ext }}^{i, \alpha}\left(\boldsymbol{\Phi}_{n-1 / 2}\right)-\boldsymbol{f}_{i n t}^{i, \alpha}\left(\boldsymbol{\Phi}_{n-1 / 2}\right)\right), \\
\tilde{\boldsymbol{v}}^{i, \alpha} & =\boldsymbol{v}_{n}^{i, \alpha}+\Delta t \tilde{\boldsymbol{a}}^{i, \alpha} \\
\tilde{\boldsymbol{\varphi}}^{i, \alpha} & =\boldsymbol{\varphi}_{n}^{i, \alpha}+\Delta t \tilde{\boldsymbol{v}}^{i, \alpha}
\end{aligned}
$$

where $\tilde{\boldsymbol{f}}_{\text {ext }}^{i, \alpha}$ refers to the nodal external force defined in Eq. (3.26), but ignoring the contributions due to contact interactions. We note that, as in the standard central difference method, the predictors $\tilde{\varphi}^{i, \alpha}$ and $\tilde{\boldsymbol{v}}^{i, \alpha}$ refer to quantities at time $t_{n+1}$ while $\tilde{\boldsymbol{a}}^{i, \alpha}$ is an approximation to the acceleration at time $t_{n+1 / 2}$.

2) If contact occurs update predicted quantities, see Sec 3.4

3) Else

$$
\left(\boldsymbol{\varphi}_{n+1}^{i, \alpha}, \boldsymbol{v}_{n+1}^{i, \alpha}, \boldsymbol{a}_{n+1 / 2}^{i, \alpha}\right)=\left(\tilde{\boldsymbol{\varphi}}^{i, \alpha}, \tilde{\boldsymbol{v}}^{i, \alpha}, \tilde{\boldsymbol{a}}^{i, \alpha}\right) .
$$

In most meshless methods, since the delta Kronecker property is not verified by the shape functions, the displacement, the velocity and the acceleration of a point is not equal to its nodal value but instead is a combination of nodal values in its neighbourhood. This complicates the enforcement of the boundary conditions and in the context of contact the update of velocities and positions. Before concluding this section, we address those meshless methods that are amenable to implementation with this type of predictor-corrector contact algorithm, namely NEM and Max-ent, methods that verify the Delta kronecker property.

\subsection{Explicit Contact}

Contact simulation is one of the cornerstones in Computational Solid Mechanics, one that is almost invariably used for complex problems, mainly in dynamics. Its ubiquitousness and the shortcomings arising from its non-smooth nature have fostered the publication of many different algorithmic approaches. There are many reviews [93]-[95] and monographs [85], [96] that classify the numerous works available on the topic, and affirm the relevance and difficulty of the problem, as well as the wide variety of numerical approaches for its approximation.

In particular, dynamic contact is one of the main problems to be faced in nonlinear mechanics, including those involving ballistics, crashworthiness, metal forming, rolling, etc. The diversity of applications has resulted in a wide range of algorithms that can be classified according to different criteria. In this chapter we focus on purely Lagrangian descriptions for solid bodies, where most of the literature is available (see, among many others, [37], [86], [87], [89], [97]-[100]). 
One of the main key points in the numerical treatment of contact is the overlap detection. In problems with multiple bodies, this step might be computationally expensive, and optimized search algorithms are essential for large scale applications [37], [100]. In these cases, moreover, parallel algorithms have been proposed to alleviate the overhead due to contact [86], [101]-[103].

Methods based on master-slave contact approaches are in their nature intrinsically asymmetrical, due to the different roles played by the master and slave surfaces. A straightforward solution to avoid such a difference is to define twice each interacting pair, exchanging the master/slave roles in each of them. In this chapter we refrain from such a symmetrization, motivated by the potential application of the methods developed herein to numerical models that combine finite element and meshfree discretizations. Thus having an asymmetric interaction. This type of models is usually employed together with explicit codes to calculate the solution of problems involving bodies that are severely distorted or even fragmented under high relative speeds of the colliding bodies. This is the case of ballistics, mentioned before, but also crash simulations, structures under explosive loading, etc. In these conditions, it is often advantageous to employ a mixed spatial discretization, one in which finite element models interact with meshfree bodies. Using such a combination, the meshfree parts can be employed to represent the components that are more severely distorted, while the finite element subdomains can model the rest, combining the advantages of meshfree methods to accommodate large distortions, and the efficiency of finite elements. From the viewpoint of contact, however, the key issue is not whether the interacting bodies are discretized with finite elements or a meshfree method, but rather if the boundary is well defined, as in a finite element mesh, but not in a meshfree or particle representation. In this work we will use examples that combine the two types of spatial discretizations, but the method is directly applicable to models based only on finite elements, where the asymmetry of the contact interaction could be reversed by defining twice the interacting pair, if desired, as in other standard implementations.

\subsubsection{Contact detection}

Contact modeling requires first to detect the overlap of bodies in the predicted solution. Obviously, if there is no overlap, this predicted solution needs not be corrected and the integration step can proceed as in free body dynamics.

In this work we put the focus on the modeling and simulation of contact interactions between bodies discretized with a mesh structure and others without it, the so-called meshless bodies. Thus, for the sake of simplicity, we consider next only the analysis of the potential contact between a body $\mathcal{B}_{i} \in \mathcal{F}$ and a second one $\mathcal{B}_{j} \in \mathcal{M}$ as depicted in Fig. 3.5. We assume also for simplicity that the body $\mathcal{B}_{i}$ will not undergo cracking or will break during the simulation, and thus its boundary remains unchanged and invariably defined.

We note that only $\mathcal{B}_{i}$ has a well-defined boundary being the proper reference to detect penetration, and thus contact detection algorithms between these two bodies are not symmetric. On $\mathcal{B}_{i}$ we can hence define the skin as the element set

$$
\mathcal{S}_{i}=\bigcup_{e_{i}^{\alpha} \cap \partial \mathcal{B}_{i} \neq \emptyset} e_{i}^{\alpha}
$$

with nodes

$$
\Sigma_{i}=\mathcal{N}_{i} \cap \mathcal{S}_{i}
$$



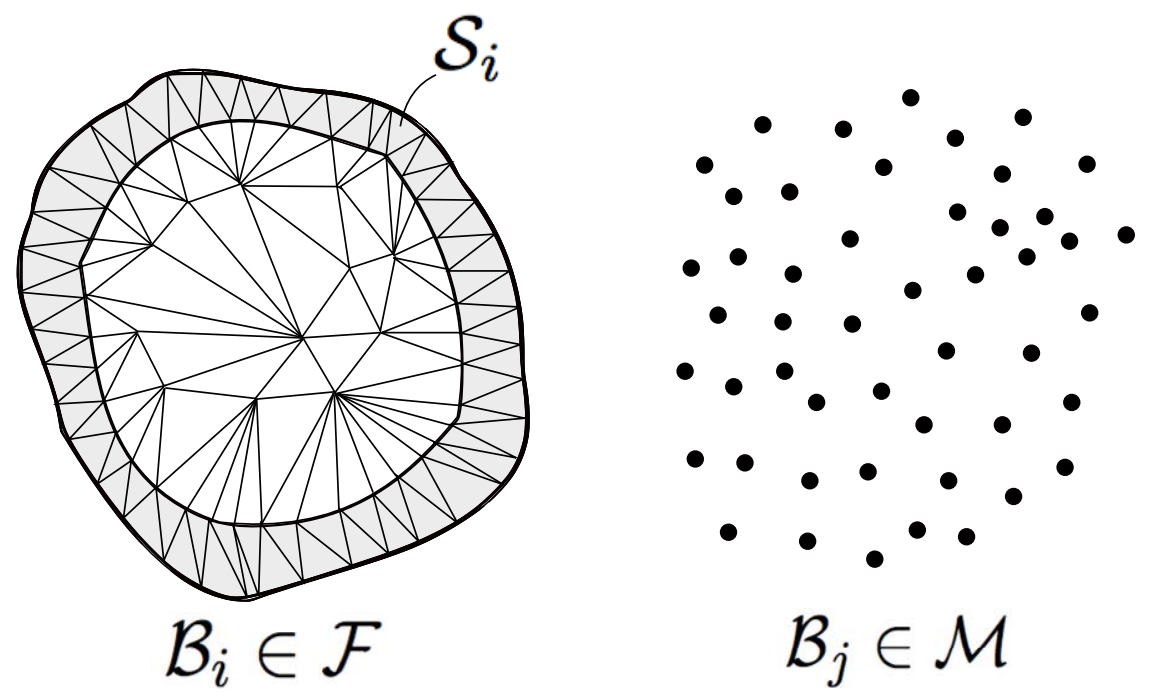

FIGURE 3.5: The two types of bodies considered are meshed (left) and meshfree (right). Bodies with a mesh have a well-defined skin of elements intersecting the boundary.

From the computational viewpoint it is important to remark that the skin typically contains far fewer elements that the body $\mathcal{B}_{i}$ itself.

The skin will be used to track potential penetrations of the meshfree body into the finite element body. To ensure that the predicted positions $\tilde{\varphi}^{j, \beta}$ do not fall within the body $\mathcal{B}_{i}$ and beyond its skin, we must restrict the time step size to be

$$
\Delta t=\min \left(\Delta t_{C F L}, \frac{h_{i}}{V_{i}+V_{j}}\right) .
$$

In this expression $\Delta t_{C F L}$ is the time step size as dictated by the CFL condition in the deformable bodies, $h_{i}$ is the minimum element size in the skin $\mathcal{S}_{i}$, and $V_{i}, V_{j}$ are the largest moduli of the velocity vectors in the two contacting bodies.

Thus one can ensure that when the time step size in the integration scheme is selected according to Eq. (3.32), the predicted position of the node $n_{j}^{\beta} \in \mathcal{N}_{j}$ can be either outside the $\operatorname{skin}$ of $\mathcal{B}_{i}$, on its surface, or inside it. But $n_{j}^{\beta}$ will not be beyond the skin. In the first two situations there is no violation of the inter-penetrability constraint, and thus the predicted position, velocity, and acceleration of the nodes $n_{i}^{\alpha} \in \mathcal{N}_{i}$ and $n_{j}^{\beta} \in \mathcal{N}_{j}$ can be taken as the correct one for the update and thus

$$
\begin{aligned}
& \left(\boldsymbol{\varphi}_{n+1}^{i, \alpha}, \boldsymbol{v}_{n+1}^{i, \alpha}, \boldsymbol{a}_{n+1 / 2}^{i, \alpha}\right)=\left(\tilde{\boldsymbol{\varphi}}^{i, \alpha}, \tilde{\boldsymbol{v}}^{i, \alpha}, \tilde{\boldsymbol{a}}^{i, \alpha}\right) \\
& \left(\boldsymbol{\varphi}_{n+1}^{j, \beta}, \boldsymbol{v}_{n+1}^{j, \beta}, \boldsymbol{a}_{n+1 / 2}^{j, \beta}\right)=\left(\tilde{\boldsymbol{\varphi}}^{j, \beta}, \tilde{\boldsymbol{v}}^{j, \beta}, \tilde{\boldsymbol{a}}^{j, \beta}\right) .
\end{aligned}
$$

\subsubsection{Correction geometry}

We study now the correction update for the nodes $n_{j}^{\beta} \in \mathcal{N}_{j}$, with $\mathcal{B}_{j} \in \mathcal{M}$, and $n_{i}^{\alpha} \in$ $\Sigma_{i}$ when the predictor update has placed $n_{j}^{\beta}$ inside the skin $\mathcal{S}_{i}$, that is, $\tilde{\varphi}^{j, \beta} \in \boldsymbol{\varphi}_{i}\left(\mathcal{S}_{i}\right)$. In such situations we need, first, to define a correction direction $\boldsymbol{n}$, with $|\boldsymbol{n}|=1$, which will be used to move the predicted position away from the interior of $\mathcal{B}_{i}$. However, the definition of such vector is not trivial because it must be defined for convex and concave polygonal bodies, and certain obvious definitions, such as the 


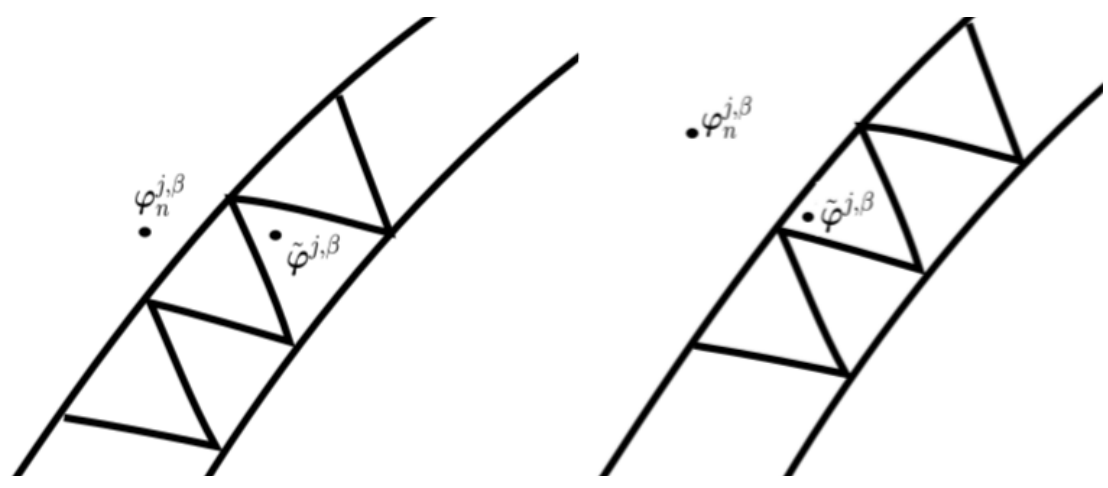

FIGURE 3.6: Two types of skin penetration in plane problems.

direction towards the closest point projection on the boundary, might not be welldefined.

For a general meshed domain $\mathcal{B}_{i}$, if the node $n_{j}^{\beta} \in \mathcal{N}_{j}$, with $\mathcal{B}_{j} \in \mathcal{M}$, at its predicted position is inside $\varphi_{i}\left(\mathcal{S}_{i}\right)$, then there must exist at least one element $e_{i}^{\alpha} \in \mathcal{S}_{i}$ such that $n_{j}^{\beta} \in e_{i}^{\alpha}$ (See Fig. 3.6). For three dimensional problems, the element $e_{i}^{\alpha}$ will have a face, an edge, or a vertex on $\partial \mathcal{B}_{i}$, and the computation of the correction direction will be done differently in each of the three situations.

The correction that needs to be performed after a penetration has been detected can be split in two steps. First, the node in the meshfree body must be moved to the surface of the skin to avoid interpenetration. Second, an exchange of linear momentum must be accounted for between this node and those in the element $e_{i}^{\alpha}$.

We address first the position correction. For any finite element body, be it convex or concave, we define the corrected position to be

$$
\boldsymbol{\varphi}_{n+1}^{j, \beta}=\arg \min _{\boldsymbol{x} \in e_{i}^{\alpha} \cap \partial \mathcal{B}_{i}}\left|\tilde{\varphi}^{j, \beta}-\boldsymbol{x}\right|,
$$

noting that, for simplicial meshes, the set $e_{i}^{\alpha} \cap \partial \mathcal{B}_{i}$, which might be a triangle, and edge, or a vertex, is always convex, and thus the minimization problem in (3.34) has always a unique solution. If the finite element mesh of the body $\mathcal{B}_{i}$ is not simplicial, this minimization might have more than one solution. In this case the corrected position is taken to be any of them.

Once the corrected position of the node has been obtained, an exchange of momentum is applied onto this same node and the nodes of the element $e_{i}^{\alpha}$. For that, a normal exchange direction needs to be defined which, irrespective of the smoothness of the surface at the projection point can always be defined as

$$
\boldsymbol{n}=\frac{\boldsymbol{\varphi}_{n+1}^{j, \beta}-\tilde{\varphi}^{j, \beta}}{\left|\boldsymbol{\varphi}_{n+1}^{j, \beta}-\tilde{\varphi}^{j, \beta}\right|}
$$

\subsubsection{Correction forces}

The node $n_{j}^{\beta} \in \mathcal{N}_{j}$ that has penetrated the skin and the element $e_{i}^{\alpha} \in \mathcal{B}_{i}$ must exchange linear momentum as a consequence of the impact. More specifically, we identify the set $\sigma_{i}^{\alpha}=\left\{n_{i}^{\gamma} \in e_{i}^{\alpha} \cap \partial \mathcal{B}_{i}\right\}$ of nodes in the finite element that receive an impulse due to the contact, the rest of nodes in $e_{i}^{\alpha}$ will not receive any impulse. In addition, we assume that the node $n_{j}^{\beta}$ and the nodes in $\sigma_{i}^{\alpha}$ have masses, denoted respectively as $m_{j}^{\beta}$ and $m_{i}^{\gamma}$, obtained by lumping their corresponding element or 
meshfree contributions, as explained in Section 3.3. Using these lumped masses we define the momenta of the nodes at time $t_{n+1}$ as

$$
\boldsymbol{p}_{n+1}^{j, \beta}=m_{j}^{\beta} \boldsymbol{v}_{n+1}^{j, \beta}, \quad \boldsymbol{p}_{n+1}^{i, \gamma}=m_{i}^{\gamma} \boldsymbol{v}_{n+1}^{i, \gamma},
$$

and use the notation $\tilde{\boldsymbol{p}}^{j, \beta}, \tilde{\boldsymbol{p}}^{i, \gamma}$ for the predicted values. The kinetic energy of the nodes will be denoted as $K_{n+1}^{j, \beta}$, etc., and defined as

$$
K_{n+1}^{j, \beta}=\frac{1}{2} m_{j}^{\beta}\left|\boldsymbol{v}_{n+1}^{j, \beta}\right|^{2}, \quad K_{n+1}^{i, \gamma}=\frac{1}{2} m_{i}^{\gamma}\left|\boldsymbol{v}_{n+1}^{i, \gamma}\right|^{2},
$$

again with $\tilde{K}^{j, \beta}, \tilde{K}^{i, \gamma}$ referring to the predicted values.

We now consider a frictionless contact with perfect energy restitution. Given the position and velocity of nodes $n_{j}^{\beta} \in \mathcal{B}_{j}$ and $n_{i}^{\gamma} \in \sigma_{i}^{\alpha}$ at the predictor step, and the unit vector $\boldsymbol{n}$ defined in Eq. (3.35), to compute their corrected velocities we impose the following balance equations:

$$
\begin{aligned}
\boldsymbol{p}_{n+1}^{j, \beta}-\tilde{\boldsymbol{p}}^{j, \beta} & =\lambda^{j, \beta} \boldsymbol{n}, \\
\boldsymbol{p}_{n+1}^{i, \gamma}-\tilde{\boldsymbol{p}}^{i, \gamma} & =-\lambda^{i, \gamma} \boldsymbol{n}, \\
\boldsymbol{p}_{n+1}^{j, \beta}+\sum_{\gamma \mid n_{i}^{\gamma} \in \sigma_{i}^{\alpha}} \boldsymbol{p}_{n+1}^{i, \gamma} & =\tilde{\boldsymbol{p}}^{j, \beta}+\sum_{\gamma \mid n_{i}^{\gamma} \in \sigma_{i}^{\alpha}} \tilde{\boldsymbol{p}}^{i, \gamma}, \\
K_{n+1}^{j, \beta}+\sum_{\gamma \mid n_{i}^{\gamma} \in \sigma_{i}^{\alpha}} K_{n+1}^{i, \gamma} & =\tilde{K}^{j, \beta}+\sum_{\gamma \mid n_{i}^{\gamma} \in \sigma_{i}^{\alpha}} \tilde{K}^{i, \gamma} .
\end{aligned}
$$

Eqs. $3.38 \mathrm{a}$ and $3.38 \mathrm{~b}$ ) express the changes in linear momentum in the involved nodes at impact. Eqs. (3.38c) and (3.38d) enforce the conservation of the interacting nodes linear momentum and kinetic energy, respectively. The system of equations (3.38) must be solved to find the updated velocities in the nodes and the impulses $\lambda^{j, \beta}, \lambda^{i, \gamma}$ which, if there is to be a physically realistic impact, must be nonnegative. However, this system is, in general, undetermined. To see this, let us first note that the components of the linear momenta that are orthogonal to $\boldsymbol{n}$ are conserved. Given any vector $\boldsymbol{m}$ such that $\boldsymbol{n} \cdot \boldsymbol{m}=0$, from Eqs. (3.38a) and (3.38b) we obtain

$$
\begin{aligned}
& \boldsymbol{p}_{n+1}^{j, \beta} \cdot \boldsymbol{m}=\tilde{\boldsymbol{p}}^{j, \beta} \cdot \boldsymbol{m}, \\
& \boldsymbol{p}_{n+1}^{i, \gamma} \cdot \boldsymbol{m}=\tilde{\boldsymbol{p}}^{i, \gamma} \cdot \boldsymbol{m} .
\end{aligned}
$$

Hence, if $M$ is the number of nodes in $\sigma_{i}^{\alpha}$, the system (3.38) can be reduced to a system of $M+3$ scalar equations

$$
\begin{aligned}
&\left.\boldsymbol{p}_{n+1}^{j, \beta}\right|_{\boldsymbol{n}}-\left.\tilde{\boldsymbol{p}}^{j, \beta}\right|_{\boldsymbol{n}}=\lambda^{j, \beta}, \\
&\left.\boldsymbol{p}_{n+1}^{i, \gamma}\right|_{\boldsymbol{n}}-\left.\tilde{\boldsymbol{p}}^{i, \gamma}\right|_{\boldsymbol{n}}=-\lambda^{i, \gamma}, \\
&\left.\boldsymbol{p}_{n+1}^{j, \beta}\right|_{\boldsymbol{n}}+\left.\sum_{\gamma \mid n_{i}^{\gamma} \in \sigma_{i}^{\alpha}} \boldsymbol{p}_{n+1}^{i, \gamma}\right|_{\boldsymbol{n}}=\left.\tilde{\boldsymbol{p}}^{j, \beta}\right|_{\boldsymbol{n}}+\left.\sum_{\gamma \mid n_{i}^{\gamma} \in \sigma_{i}^{\alpha}} \tilde{\boldsymbol{p}}^{i, \gamma}\right|_{\boldsymbol{n}} \\
& K_{n+1}^{j, \beta}+\sum_{\gamma \mid n_{i}^{\gamma} \in \sigma_{i}^{\alpha}} K_{n+1}^{i, \gamma}=\tilde{K}^{j, \beta}+\sum_{\gamma \mid n_{i}^{\gamma} \in \sigma_{i}^{\alpha}} \tilde{K}^{i, \gamma}
\end{aligned}
$$

where the notation $\left.(\cdot)\right|_{n}$ denotes the projection of a vector onto the direction $\boldsymbol{n}$. In this system there are $2 M+2$ unknowns, namely, the $M+1$ components of the linear 
momenta at the corrected step in the direction of $\boldsymbol{n}$, and the $M+1$ impulses. The system has a unique solution only when $M=1$, which is no other than the impact of two particles. For this case, there exists a standard solution. We propose next a contact model that provides $M-1$ additional equations and allows to solve the update for an arbitrary number of nodes in the contacting surface. First, let us note that Eq. (3.40c) can be rewritten as

$$
\lambda^{j, \beta}=\sum_{\gamma \mid n_{i}^{\gamma} \in \sigma_{i}^{\alpha}} \lambda^{i, \gamma}
$$

Then, let us define the barycentric coordinates of the projection $\varphi_{n+1}^{j, \beta}$, that is, the scalars $\chi^{i, \gamma} \geq 0$ such that

$$
\varphi_{n+1}^{j, \beta}=\sum_{\gamma \mid n_{i}^{\gamma} \in \sigma_{i}^{\alpha}} \chi^{i, \gamma} \tilde{\varphi}^{i, \gamma},
$$

with $\sum_{\gamma \mid n_{i}^{\gamma} \in \sigma_{i}^{\alpha}} \chi^{i, \gamma}=1$, noting that the projected position depends only on the predictors. In view of the well-posedness of projection (3.34) the coordinates are unique. The contact model proposed apportions the impulse $\lambda^{j, \beta}$ to the nodes on $\sigma_{i}^{\alpha}$ according to these barycentric coordinates, that is,

$$
\lambda^{i, \gamma}=\chi^{i, \gamma} \lambda^{j, \beta},
$$

providing $M-1$ additional equations that close the system (3.40).

\subsubsection{Velocity solution algorithm}

The last step in the contact update is the solution of the system of equations (3.40). A closed form expression for the impulses and velocities at the predicted step can be found as follows. An algebraic manipulation shows that the energy balance $3.40 \mathrm{~d}$ ) can be written as

$$
0=\sum_{\gamma \mid n_{i}^{\gamma} \in \sigma_{i}^{\alpha}}\left(\frac{\left(\lambda^{i, \gamma}\right)^{2}}{2 m_{i}^{\gamma}}-\lambda^{i, \gamma} \tilde{\boldsymbol{v}}^{i, \gamma} \cdot \boldsymbol{n}\right)+\frac{\left(\lambda^{j, \beta}\right)^{2}}{2 m_{j}^{\beta}}+\lambda^{j, \beta} \tilde{\boldsymbol{v}}^{j, \beta} \cdot \boldsymbol{n} .
$$

Then, using the contact law (3.43) and defining

$$
\tilde{\boldsymbol{\nu}}^{i, \alpha}=\sum_{\gamma \mid n_{i}^{\gamma} \in \sigma_{i}^{\alpha}} \chi^{i, \gamma} \tilde{\boldsymbol{v}}^{i, \gamma}, \quad \frac{1}{\mu_{i}^{\alpha}}=\sum_{\gamma \mid n_{i}^{\gamma} \in \sigma_{i}^{\alpha}} \frac{\left(\chi^{i, \gamma}\right)^{2}}{m_{i}^{\gamma}},
$$

Eq. (3.44) can be rewritten as

$$
0=\frac{\left(\lambda^{j, \beta}\right)^{2}}{2}\left(\frac{1}{\mu_{i}^{\alpha}}+\frac{1}{m_{j}^{\beta}}\right)+\lambda^{j, \beta}\left(\tilde{\boldsymbol{v}}^{j, \beta}-\tilde{\boldsymbol{\nu}}^{i, \alpha}\right) \cdot \boldsymbol{n} .
$$

This second order polynomial in $\lambda^{j, \beta}$ has a unique non-zero root

$$
\lambda^{j, \beta}=2 \frac{m_{j}^{\beta} \mu_{i}^{\alpha}}{m_{j}^{\beta}+\mu_{i}^{\alpha}}\left(\tilde{\boldsymbol{\nu}}^{i, \alpha}-\tilde{\boldsymbol{v}}^{j, \beta}\right) \cdot \boldsymbol{n},
$$

obtaining this closed form that gives the modulus and direction of the correction impulse on the node $n_{j}^{\beta}$ on the meshfree body. The corresponding impulses on the 
finite element surface are obtained using Eq. (3.43). Remarkably, Eq. (3.47) has the same structure as the standard formula that computes the change of linear momentum in the collision between two point masses. The proposed algorithm, thus, can be thought of as solving the impact between the node $n_{j}^{\beta}$ and a particle of mass $\mu_{i}^{\alpha}$ and velocity $\tilde{\boldsymbol{\nu}}^{i, \alpha}$.

Physically based arguments require that the impulses imparted to the nodes due to impact are all non-negative. In fact, for the model employed, Eq. (3.43) shows that it is sufficient to show that $\lambda^{j, \beta} \geq 0$. The inner product in Eq. (3.47) can be rewritten, with the aid of Eqs. (3.28) and (3.45), as

$$
\begin{aligned}
\boldsymbol{n} \cdot\left(\tilde{\boldsymbol{\nu}}^{i, \alpha}-\tilde{\boldsymbol{v}}^{j, \beta}\right)= & \frac{1}{\Delta t} \boldsymbol{n} \cdot\left(\sum_{\gamma \mid n_{i}^{\gamma} \in \sigma_{i}^{\alpha}} \chi^{i, \gamma} \tilde{\boldsymbol{\varphi}}^{i, \gamma}-\tilde{\boldsymbol{\varphi}}^{j, \beta}\right) \\
& -\frac{1}{\Delta t} \boldsymbol{n} \cdot\left(\sum_{\gamma \mid n_{i}^{\gamma} \in \sigma_{i}^{\alpha}} \chi^{i, \gamma} \boldsymbol{\varphi}_{n}^{i, \gamma}-\boldsymbol{\varphi}_{n}^{j, \beta}\right) .
\end{aligned}
$$

The first term on the right hand side can be further simplified to

$$
\frac{1}{\Delta t} \boldsymbol{n} \cdot\left(\sum_{\gamma \mid n_{i}^{\gamma} \in \sigma_{i}^{\alpha}} \chi^{i, \gamma} \tilde{\boldsymbol{\varphi}}^{i, \gamma}-\tilde{\boldsymbol{\varphi}}^{j, \beta}\right)=\frac{1}{\Delta t} \boldsymbol{n} \cdot\left(\boldsymbol{\varphi}_{n+1}^{j, \beta}-\tilde{\boldsymbol{\varphi}}^{j, \beta}\right)=\frac{\left|\boldsymbol{\varphi}_{n+1}^{j, \beta}-\tilde{\varphi}^{j, \beta}\right|}{\Delta t}
$$

which is, obviously, non-negative. The second term in Eq. (3.48) has an undetermined sign. When the contacting surfaces are smooth, it should be expected that the term in parenthesis does not differ much from $n$, which is computed for the predictor state, and that this second term becomes non-negative. In the case of surfaces with corners, however, this need not be the case and it might result in numerical impulses that are in the opposite direction to the one expected. This is a side effect of the explicit integration, but one that rarely takes place.

\subsubsection{Inelastic collisions}

During inelastic collisions kinetic energy is not preserved and hence Eq. (3.40d), the kinetic energy conservation, is not verified. Instead, a different relation accounting for the energy loss needs to be provided to relate the velocities of the impacting nodes. A common choice is a linear equation that determines a ratio of the relative velocities between colliding particles before and after the impulse update. Using again the two-particle impact analogy, we propose this equation to be

$$
\left(\boldsymbol{\nu}_{n+1}^{i, \alpha}-\boldsymbol{v}_{n+1}^{j, \beta}\right) \cdot \boldsymbol{n}=-e\left(\tilde{\boldsymbol{\nu}}^{i, \alpha}-\tilde{\boldsymbol{v}}^{j, \beta}\right) \cdot \boldsymbol{n},
$$

where, $e \in[0,1]$, is the coefficient of restitution and $\boldsymbol{\nu}_{n+1}^{i, \alpha}$ is defined, in analogy with the definition of equivalent predictor velocity in expression (3.45), as the vector

$$
\boldsymbol{\nu}_{n+1}^{i, \alpha}=\sum_{\gamma \mid n_{i}^{\gamma} \in \sigma_{i}^{\alpha}} \chi^{i, \gamma} \boldsymbol{v}_{n+1}^{i, \gamma}
$$


Using Eqs. (3.40a) to 3.40c), 3.43 and (3.50), the correction impulse on the node $n_{j}^{\beta}$ follows as

$$
\lambda^{j, \beta}=(1+e) \frac{m_{j}^{\beta} \mu_{i}^{\alpha}}{m_{j}^{\beta}+\mu_{i}^{\alpha}}\left(\tilde{\boldsymbol{\nu}}^{i, \alpha}-\tilde{\boldsymbol{v}}^{j, \beta}\right) \cdot \boldsymbol{n} .
$$

When $e=1$, we obtain the energy-preserving solution given by Eq. (3.47); when $e=0$, the dissipation in the projection step is maximum. To see this, let us define the dissipation $\mathcal{D}=\tilde{K}-K_{n+1}$ and use Eq. (3.46) to obtain

$$
\mathcal{D}=\lambda^{j, \beta}\left(\tilde{\boldsymbol{\nu}}^{i, \alpha}-\tilde{\boldsymbol{v}}^{j, \beta}\right) \cdot \boldsymbol{n}-\frac{\left(\lambda^{j, \beta}\right)^{2}}{2} \frac{\mu_{i}^{\alpha}+\lambda^{j, \beta}}{\mu_{i}^{\alpha} \lambda^{j, \beta}} .
$$

Taking the derivative of this equation with respect to $\lambda^{j, \beta}$, and setting it equal to zero we can deduce that the dissipation has its maximum value when the impulse is

$$
\lambda_{\max \mathcal{D}}^{j, \beta}=\frac{m_{j}^{\beta} \mu_{i}^{\alpha}}{m_{j}^{\beta}+\mu_{i}^{\alpha}}\left(\tilde{\boldsymbol{\nu}}^{i, \alpha}-\tilde{\boldsymbol{v}}^{j, \beta}\right) \cdot \boldsymbol{n},
$$

which is precisely the value obtained in Eq. (3.52) when $e=0$. As a result, the maximum value of the dissipation in a frictionless but inelastic impact is:

$$
\mathcal{D}=\frac{\mu_{i}^{\alpha} m_{j}^{\beta}}{2\left(\mu_{i}^{\alpha}+m_{j}^{\beta}\right)}\left(\left(\tilde{\boldsymbol{\nu}}^{i, \alpha}-\tilde{\boldsymbol{v}}^{j, \beta}\right) \cdot \boldsymbol{n}\right)^{2} .
$$

\subsubsection{Friction models}

Finally, we consider potential frictional effects between the contacting bodies, allowing for contact impulses with both normal and tangential components. The equations that govern the problem are similar to Eqs. (3.38), but now the impulses should not be restricted to be parallel to the direction $n$, instead they also have a component in the plane normal to $\boldsymbol{n}$. The general discrete balance equations are thus

$$
\begin{aligned}
\boldsymbol{p}_{n+1}^{j, \beta}-\tilde{\boldsymbol{p}}^{j, \beta} & =\boldsymbol{i}^{j, \beta}, \\
\boldsymbol{p}_{n+1}^{i, \gamma}-\tilde{\boldsymbol{p}}^{i, \gamma} & =\boldsymbol{i}^{i, \gamma}, \\
\boldsymbol{p}_{n+1}^{j, \beta}+\sum_{\gamma \mid n_{i}^{\gamma} \in \sigma_{i}^{\alpha}} \boldsymbol{p}_{n+1}^{i, \gamma} & =\tilde{\boldsymbol{p}}^{j, \beta}+\sum_{\gamma \mid n_{i}^{\gamma} \in \sigma_{i}^{\alpha}} \tilde{\boldsymbol{p}}^{i, \gamma}, \\
\left(\boldsymbol{\nu}_{n+1}^{i, \alpha}-\boldsymbol{v}_{n+1}^{j, \beta}\right) \cdot \boldsymbol{n} & =-e\left(\tilde{\boldsymbol{\nu}}^{i, \alpha}-\tilde{\boldsymbol{v}}^{j, \beta}\right) \cdot \boldsymbol{n},
\end{aligned}
$$

where $\boldsymbol{i}^{j, \beta}, \boldsymbol{i}^{i, \gamma}$ are now the impulses on the nodes. The solution of this system can be more easily obtained if the first three equations are projected onto the direction $\boldsymbol{n}$, and later solved in the plane perpendicular to this vector. The three projections onto $n$, together with $\mathrm{Eq}$ (3.56d), are precisely the ones studied in Section 3.4.5 and yield the velocity updates in the $\boldsymbol{n}$ direction.

The velocity updates in the plane orthogonal to $\boldsymbol{n}$ require additional model equations. To introduce such a model, let us first define the projection

$$
\Pi(\boldsymbol{v})=\boldsymbol{v}-\left.\boldsymbol{v}\right|_{n} \boldsymbol{n}=\boldsymbol{v}-(\boldsymbol{v} \cdot \boldsymbol{n}) \boldsymbol{n},
$$


mapping an arbitrary vector $\boldsymbol{v}$ onto the orthogonal complement to $\boldsymbol{n}$, and let $\tilde{\boldsymbol{\omega}}^{i, \alpha}$ be the weighted velocity

$$
\tilde{\boldsymbol{\omega}}^{i, \alpha}=\frac{1}{M_{i}^{\alpha}} \sum_{\gamma \mid n_{i}^{\gamma} \in \sigma_{i}^{\alpha}} m_{i}^{\gamma} \tilde{\boldsymbol{v}}^{i, \gamma},
$$

with $M_{i}^{\alpha}$ being the total mass of the nodes in $\sigma_{i}^{\alpha}$. In the orthogonal complement to $\boldsymbol{n}$, let us define the unit vectors

$$
\boldsymbol{\tau}=\frac{\Pi\left(\tilde{\boldsymbol{v}}^{j, \beta}-\tilde{\boldsymbol{\omega}}^{i, \alpha}\right)}{\left|\Pi\left(\tilde{\boldsymbol{v}}^{j, \beta}-\tilde{\boldsymbol{\omega}}^{i, \alpha}\right)\right|}, \quad \boldsymbol{\xi}=\boldsymbol{\tau} \times \boldsymbol{n} .
$$

To model the frictional effects, first, we decompose the nodal impulses on the tangential directions $\boldsymbol{\xi}$ and $\boldsymbol{\tau}$ as in:

$$
\Pi\left(\boldsymbol{i}^{j, \beta}\right)=-\eta^{j, \beta} \boldsymbol{\tau}-\rho^{j, \beta} \boldsymbol{\xi}, \quad \Pi\left(\boldsymbol{i}^{i, \gamma}\right)=\eta^{i, \gamma} \boldsymbol{\tau}+\rho^{i, \gamma} \boldsymbol{\xi},
$$

where $\eta^{j, \beta}, \rho^{j, \beta}$ are nonnegative scalars and $\eta^{i, \gamma}, \rho^{i, \gamma}$ distribute the impulse among the contacting nodes. Second, some additional relations must be provided among the tangential and normal impulses. Assuming a simple Coulomb law with constant friction coefficient $\mu$, the tangential impulse on the node must satisfy

$$
0 \leq \sqrt{\left(\eta^{j, \beta}\right)^{2}+\left(\rho^{j, \beta}\right)^{2}} \leq \mu \lambda^{j, \beta}
$$

Finally, the two possible contact types, namely stick or slip contact, must be distinguished, and modeled accordingly.

During stick contact there is no sliding in the tangential direction between the node $n_{j}^{\beta}$ and the surface $\sigma_{i}^{\alpha}$, that is,

$$
\Pi\left(\boldsymbol{v}_{n+1}^{i, \gamma}\right)=\Pi\left(\boldsymbol{v}_{n+1}^{j, \beta}\right),
$$

for all nodes $n_{i}^{\gamma} \in \sigma_{i}^{\alpha}$. In this situation, the system of equations that governs the tangential contact update is

$$
\begin{aligned}
\Pi\left(\boldsymbol{p}_{n+1}^{j, \beta}-\tilde{\boldsymbol{p}}^{j, \beta}\right) & =-\eta^{j, \beta} \boldsymbol{\tau}-\rho^{j, \beta} \boldsymbol{\xi}, \\
\Pi\left(\boldsymbol{p}_{n+1}^{i, \gamma}-\tilde{\boldsymbol{p}}^{i, \gamma}\right) & =\eta^{i, \gamma} \boldsymbol{\tau}+\rho^{i, \gamma} \boldsymbol{\xi}, \\
\Pi\left(\boldsymbol{p}_{n+1}^{j, \beta}\right)+\sum_{\gamma \mid n_{i}^{\gamma} \in \sigma_{i}^{\alpha}} \Pi\left(\boldsymbol{p}_{n+1}^{i, \gamma}\right) & =\Pi\left(\tilde{\boldsymbol{p}}^{j, \beta}\right)+\sum_{\gamma \mid n_{i}^{\gamma} \in \sigma_{i}^{\alpha}} \Pi\left(\tilde{\boldsymbol{p}}^{i, \gamma}\right), \\
\frac{\Pi\left(\boldsymbol{p}_{n+1}^{j, \beta}\right)}{m_{j}^{\beta}} & =\frac{\Pi\left(\boldsymbol{p}_{n+1}^{i, \gamma}\right)}{m_{i}^{\gamma}} .
\end{aligned}
$$

A simple algebraic manipulation gives

$$
\eta^{j, \beta}=\frac{m_{j}^{\beta} M_{i}^{\alpha}}{m_{j}^{\beta}+M_{i}^{\alpha}}\left(\tilde{\boldsymbol{v}}^{j, \beta}-\tilde{\boldsymbol{\omega}}^{i, \alpha}\right) \cdot \boldsymbol{\tau},
$$

which is always non-negative and $\rho^{j, \beta}$

$$
\rho^{j, \beta}=\frac{m_{j}^{\beta} M_{i}^{\alpha}}{m_{j}^{\beta}+M_{i}^{\alpha}}\left(\tilde{\boldsymbol{v}}^{j, \beta}-\tilde{\boldsymbol{\omega}}^{i, \alpha}\right) \cdot \boldsymbol{\xi}=0,
$$


due to the orthonormality of $\tau$ and $\boldsymbol{\xi}$. Combining the last expressions with Eq. (3.52), the stick condition can be rewritten as

$$
\frac{M_{i}^{\alpha}}{m_{j}^{\beta}+M_{i}^{\alpha}}\left(\tilde{\boldsymbol{v}}^{j, \beta}-\tilde{\boldsymbol{\omega}}^{i, \alpha}\right) \cdot \boldsymbol{\tau} \leq \frac{\mu_{i}^{\alpha}}{m_{j}^{\beta}+\mu_{i}^{\alpha}} \mu(1+e)\left(\tilde{\boldsymbol{\nu}}^{i, \alpha}-\tilde{\boldsymbol{v}}^{j, \beta}\right) \cdot \boldsymbol{n},
$$

which, conveniently, only depends on quantities evaluated at the predictor step. Using it, we can decide, explicitly, whether the tangential motion during the update is going to involve sliding or not.

If, however, slip takes place, $\eta^{j, \beta}=\mu \lambda^{j, \beta}$, and the model is completed by apportioning the impulse on the element nodes proportionally to their masses, that is,

$$
\eta^{i, \gamma}=\frac{m_{i}^{\gamma}}{M_{i}^{\alpha}} \eta^{j, \beta}, \quad \rho^{i, \gamma}=\frac{m_{i}^{\gamma}}{M_{i}^{\alpha}} \rho^{j, \beta}
$$

and by continuity between stick and slip contact we assume $\rho^{j, \beta}=0$.

Finally we want to conclude this section looking at the balance of energy during the projection step, and verify that indeed the update defined for frictional contact in the tangential direction produces non-negative dissipation. To see this, we first note that the kinetic energy of the contacting entities at the corrected step can be expressed as a function of the velocities at the predicted step and impulses in the normal and tangential direction. Combining the velocity updates of Eqs. (3.38) and (3.60) we can write the kinetic energy at the corrected step as

$$
\begin{aligned}
K_{n+1} & =\sum_{\gamma \mid n_{i}^{\gamma} \in \sigma_{i}^{\alpha}} \frac{m_{i}^{\gamma}}{2}\left|\tilde{\boldsymbol{v}}^{i, \gamma}-\frac{\chi^{i, \gamma} \lambda^{j, \beta}}{m_{i}^{\gamma}} \boldsymbol{n}+\frac{\eta^{i, \gamma}}{m_{i}^{\gamma}} \boldsymbol{\tau}+\frac{\rho^{i, \gamma}}{m_{i}^{\gamma}} \boldsymbol{\xi}\right|^{2} \\
& +\frac{m_{j}^{\beta}}{2}\left|\tilde{\boldsymbol{v}}^{j, \beta}+\frac{\lambda^{j, \beta}}{m_{j}^{\beta}} \boldsymbol{n}-\frac{\eta^{j, \beta}}{m_{j}^{\beta}} \boldsymbol{\tau}-\frac{\rho^{j, \beta}}{m_{j}^{\beta}} \boldsymbol{\xi}\right|^{2} .
\end{aligned}
$$

For the case of stick frictional contact, the dissipation $\mathcal{D}=\tilde{K}-K_{n+1}$ can be found to be

$$
\begin{aligned}
\mathcal{D}= & \lambda^{j, \beta} \tilde{\boldsymbol{\nu}}^{i, \alpha} \cdot \boldsymbol{n}-\frac{\left(\lambda^{j, \beta}\right)^{2}}{2 \mu_{i}^{\alpha}}-\frac{\left(\lambda^{j, \beta}\right)^{2}}{2 m_{j}^{\beta}}-\lambda^{j, \beta} \tilde{\boldsymbol{v}}^{j, \beta} \cdot \boldsymbol{n} \\
& -\sum_{\gamma \mid n_{i}^{\gamma} \in \sigma_{i}^{\alpha}} \eta^{i, \gamma} \tilde{\boldsymbol{v}}^{i, \gamma} \cdot \boldsymbol{\tau}-\frac{1}{2} \sum_{\gamma \mid n_{i}^{\gamma} \in \sigma_{i}^{\alpha}} \frac{\left(\eta^{i, \gamma}\right)^{2}}{m_{i}^{\gamma}}-\frac{\left(\eta^{j, \beta}\right)^{2}}{2 m_{j}^{\beta}}+\eta^{j, \beta} \tilde{\boldsymbol{v}}^{j, \beta} \cdot \boldsymbol{\tau} \\
& -\sum_{\gamma \mid n_{i}^{\gamma} \in \sigma_{i}^{\alpha}} \rho^{i, \gamma} \tilde{\boldsymbol{v}}^{i, \gamma} \cdot \boldsymbol{\xi}-\frac{1}{2} \sum_{\gamma \mid n_{i}^{\gamma} \in \sigma_{i}^{\alpha}} \frac{\left(\rho^{i, \gamma}\right)^{2}}{m_{i}^{\gamma}}
\end{aligned}
$$

where the first line of the right-hand side of the equation above corresponds to Eq. (3.53) and is non-negative for the value $\lambda^{j, \beta}$ in Eq. (3.52). The rest of the righthand side is the energy dissipated due to the frictional contact, namely $\mathcal{D}_{\tau}+\mathcal{D}_{\xi}$, and can be elaborated to

$$
\begin{aligned}
\mathcal{D}_{\boldsymbol{\tau}}= & \sum_{\gamma \mid n_{i}^{\gamma} \in \sigma_{i}^{\alpha}} \frac{1}{2} m_{i}^{\gamma}\left(\tilde{\boldsymbol{v}}^{i, \gamma} \cdot \boldsymbol{\tau}\right)^{2}-\frac{1}{2} M_{i}^{\alpha}\left(\tilde{\boldsymbol{v}}^{j, \beta} \cdot \boldsymbol{\tau}\right)^{2} \\
& -\frac{M_{i}^{\alpha}\left(\eta^{j, \beta}\right)^{2}}{2\left(m_{j}^{\beta}\right)^{2}}+\frac{\eta^{j, \beta} M_{i}^{\alpha}}{m_{j}^{\beta}}\left(\tilde{\boldsymbol{v}}^{j, \beta} \cdot \boldsymbol{\tau}\right)+\eta^{j, \beta}\left(\tilde{\boldsymbol{v}}^{j, \beta} \cdot \boldsymbol{\tau}\right)-\frac{\left(\eta^{j, \beta}\right)^{2}}{2 m_{j}^{\beta}},
\end{aligned}
$$


where we have employed that

$$
\eta^{i, \gamma}=m_{i}^{\gamma}\left(\frac{-\eta^{j, \beta}}{m_{j}^{\beta}}+\tilde{\boldsymbol{v}}^{j, \beta} \cdot \boldsymbol{\tau}-\tilde{\boldsymbol{v}}^{i, \gamma} \cdot \boldsymbol{\tau}\right) .
$$

By Jensen's inequality,

$$
\frac{M_{i}^{\alpha}}{2}\left(\tilde{\boldsymbol{\omega}}^{i, \alpha} \cdot \boldsymbol{\tau}\right)^{2} \leq \sum_{\gamma \mid n_{i}^{\gamma} \in \sigma_{i}^{\alpha}} \frac{1}{2} m_{i}^{\gamma}\left(\tilde{\boldsymbol{v}}^{i, \gamma} \cdot \boldsymbol{\tau}\right)^{2},
$$

and thus, using Eq. (3.64),

$$
\mathcal{D}_{\boldsymbol{\tau}} \geq \frac{m_{j}^{\beta} M_{i}^{\alpha}}{2\left(m_{j}^{\beta}+M_{i}^{\alpha}\right)}\left(\left(\tilde{\boldsymbol{\omega}}^{i, \alpha}-\tilde{\boldsymbol{v}}^{j, \beta}\right) \cdot \boldsymbol{\tau}\right)^{2},
$$

which is non-negative. Similarly we can obtain that $\mathcal{D}_{\boldsymbol{\xi}} \geq 0$ where we have employed that

$$
\rho^{i, \gamma}=m_{i}^{\gamma}\left(\tilde{\boldsymbol{v}}^{j, \beta} \cdot \boldsymbol{\xi}-\tilde{\boldsymbol{v}}^{i, \gamma} \cdot \boldsymbol{\xi}\right),
$$

using Eq. 3.63). For the case of slip contact, the dissipation in the correction step is now

$$
\begin{aligned}
\mathcal{D}= & \lambda^{j, \beta} \tilde{\boldsymbol{\nu}}^{i, \alpha} \cdot \boldsymbol{n}-\mu \lambda^{j, \beta} \tilde{\boldsymbol{\omega}}^{i, \alpha} \cdot \boldsymbol{\tau}-\lambda^{j, \beta} \tilde{\boldsymbol{v}}^{j, \beta} \cdot \boldsymbol{n} \\
& +\mu \lambda^{j, \beta} \tilde{\boldsymbol{v}}^{j, \beta} \cdot \boldsymbol{\tau}-\frac{\left(\lambda^{j, \beta}\right)^{2}}{2}\left(\frac{1}{\mu_{i}^{\alpha}}+\frac{\mu^{2}}{M_{i}^{\alpha}}+\frac{1+\mu^{2}}{m_{j}^{\beta}}\right) .
\end{aligned}
$$

This is a parabola on the non-negative scalar $\lambda^{j, \beta}$, and evaluates to a non-negative dissipation as long as $\lambda^{j, \beta} \in\left(0, \lambda_{\mathcal{D}=0}^{j, \beta}\right)$ where $\lambda_{\mathcal{D}=0}^{j, \beta}$ is

$$
\lambda_{\mathcal{D}=0}^{j, \beta}=2 \frac{\left(\tilde{\boldsymbol{\nu}}^{i, \alpha}-\tilde{\boldsymbol{v}}^{j, \beta}\right) \cdot \boldsymbol{n}+\mu\left(\tilde{\boldsymbol{v}}^{j, \beta}-\tilde{\boldsymbol{\omega}}^{i, \alpha}\right) \cdot \boldsymbol{\tau}}{\frac{1}{\mu_{i}^{\alpha}}+\frac{\mu^{2}}{M_{i}^{\alpha}}+\frac{1+\mu^{2}}{m_{j}^{\beta}}} .
$$

Employing Eq. (3.66) to find the limit value, the dissipation can be re-written as

$$
\lambda_{\mathcal{D}=0}^{j, \beta}=2 \frac{\left(\tilde{\boldsymbol{\nu}}^{i, \alpha}-\tilde{\boldsymbol{v}}^{j, \beta}\right) \cdot \boldsymbol{n}}{\frac{1}{\mu_{i}^{\alpha}}+\frac{\mu^{2}}{M_{i}^{\alpha}}+\frac{1+\mu^{2}}{m_{j}^{\beta}}}\left(1+\mu^{2}(1+e) \frac{\mu_{i}^{\alpha}\left(m_{j}^{\beta}+M_{i}^{\alpha}\right)}{M_{i}^{\alpha}\left(m_{j}^{\beta}+\mu_{i}^{\alpha}\right)}\right) .
$$

But this limit value can be bounded from below since

$$
\lambda_{\mathcal{D}=0}^{j, \beta} \geq 2 \frac{\left(\tilde{\boldsymbol{\nu}}^{i, \alpha}-\tilde{\boldsymbol{v}}^{j, \beta}\right) \cdot \boldsymbol{n}}{\frac{1}{\mu_{i}^{\alpha}}+\frac{1}{m_{j}^{\beta}}} \geq(1+e) \frac{\mu_{i}^{\alpha} m_{j}^{\beta}}{\mu_{i}^{\alpha}+m_{j}^{\beta}}\left(\tilde{\boldsymbol{\nu}}^{i, \alpha}-\tilde{\boldsymbol{v}}^{j, \beta}\right) \cdot \boldsymbol{n}=\lambda^{j, \beta},
$$

where the last identity follows Eq. (3.52). We conclude that $0 \leq \lambda^{j, \beta} \leq \lambda_{\mathcal{D}=0}^{j, \beta}$ and thus the energy dissipation is also non-negative in the slip case.

\subsubsection{Relation of the proposed algorithm with existing methods}

The most commonly employed methods for dealing with contacting surfaces in explicit dynamics start from the definition of a gap function $g$ that gauges the distance between interacting bodies. Numerical methods are designed to keep this function 
larger than or equal to zero and, potentially, to impose forces in the direction of its gradient in order to prevent it from becoming negative (see, for example, [96]).

Numerical methods of the type referred to impose the balance of linear momentum as in

$$
\boldsymbol{p}_{n+1}^{a, b}-\tilde{\boldsymbol{p}}^{a, b}=\lambda \frac{\partial g\left(\mathbf{\Phi}_{n}\right)}{\partial \boldsymbol{\varphi}^{a, b}} \quad a=\{i, j\}, b=\left\{\gamma \mid n_{i}^{\gamma} \in \sigma_{i}^{\alpha}, \beta\right\},
$$

with $\lambda=\kappa g\left(\boldsymbol{\Phi}_{n}\right)$, and $\kappa$ a penalty constant. To reveal the relation between these methods and the one presented in this work let us consider the gap function

$$
g\left(\left\{\boldsymbol{\varphi}^{i, \gamma}\right\}_{\gamma \mid n_{i}^{\gamma} \in \sigma_{i}^{\alpha}}, \boldsymbol{\varphi}^{j, \beta}\right)=\left\langle\boldsymbol{n}, \boldsymbol{\varphi}^{j, \beta}-\sum_{\gamma \mid n_{i}^{\gamma} \in \sigma_{i}^{\alpha}} \chi^{i, \gamma} \boldsymbol{\varphi}^{i, \gamma}\right\rangle
$$

where $\chi^{i, \gamma}$ are the scalar coefficients defined in Eq. (3.42), with node $n_{\beta}^{j}$ in the meshfree body and nodes $n_{i}^{\gamma}$ on the face $\sigma_{i}^{\alpha}$ of a finite element mesh.

First, we note that the sum over nodes on the element face $\sigma_{i}^{\alpha}$ is a convex combination that results in a point inside the face. This shows that the function (3.80) is just measuring the (signed) distance of the node $n_{\beta}^{j}$ to the surface $\sigma_{i}^{\alpha}$, and thus it seems a potentially useful gap function. But second, a straightforward calculation reveals that the impulse equations (3.79) are precisely Eq. (3.38a), for the node $n_{j}^{\beta}$, and Eqs. $3.38 \mathrm{~b}$ for the nodes $n_{i}^{\gamma} \in \sigma_{i}^{\alpha}$. Hence we conclude that the algorithms developed herein, at least for the normal component, are just a particular instance of gap-driven contact methods, in which the gap function is given by Eq. (3.80). Finally, we note that the method proposed in the current work replaces the penalty form of the impulse $\lambda$ with the prediction/correction algorithm.

Before introducing some benchmarks in the next section, we refer to the flowchart in Fig. 3.7 as a summary of the different steps to perform contact using this algorithm.

\subsubsection{Contact benchmarks}

\section{Double elastic bar impact}

We now analyze the elastic impact of two identical rods against each other. The cylinders have radius and length equal to $3.2 \mathrm{~mm}$ and $32.4 \mathrm{~mm}$, respectively. One of the rods is discretized with finite elements and the other is discretized with a meshfree method, with symmetry conditions employed in both cases we restrict the analysis to one-quarter of the domain. The finite element and the meshfree bodies use, respectively, 798 and 1093 nodes. The material of the rods is represented with a hyperelastic neo-hookean model with Young's modulus $E=117 \mathrm{GPa}$, Poisson's ratio $\nu=0.35$, and density $8930 \mathrm{~kg} / \mathrm{m}^{3}$. The rods are launched at $113.5 \mathrm{~m} / \mathrm{s}$ against each other and compression waves are originated in both bodies when they come into contact. These waves travel back as release waves causing the bodies to separate, eventually. See Fig. 3.8 for an illustration.

This example shows that the proposed contact algorithm is able to deal with high velocity impacts of finite elements/meshfree discretizations in a robust way, without the need to adjust any penalty parameter. As a result, not only the definition of the contact properties is simplified, but also it has no influence whatsoever in the critical time step size. 


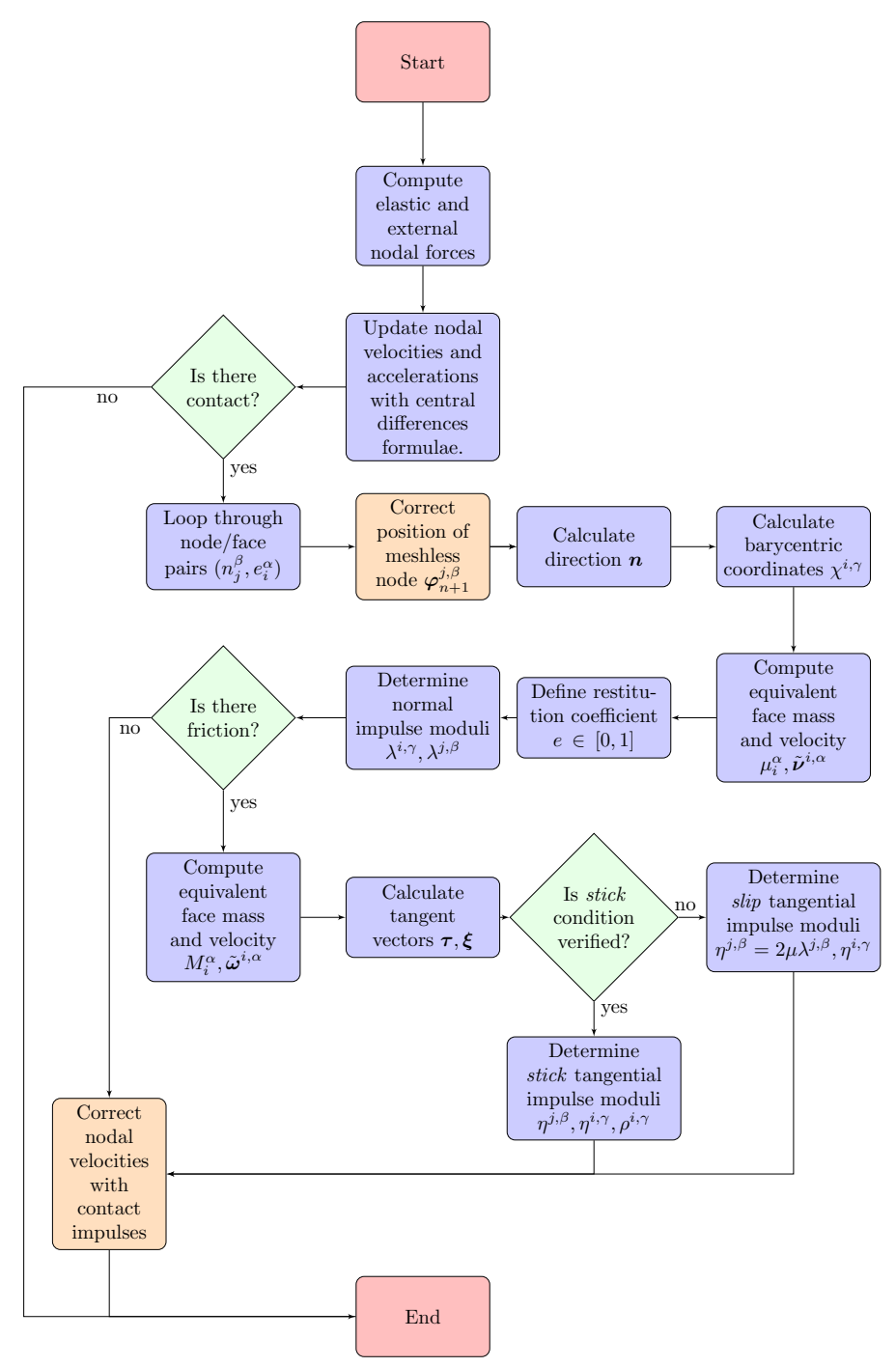

FIGURE 3.7: Flowchart for the contact algorithm.

\section{High velocity impact of a sphere onto a deformable plate}

As hinted at in the introduction, high velocity impacts are one of the most common applications of combined finite elements/meshfree methods, especially when one of the interacting bodies (typically the impactor) suffers large distortions, even fracture. In the following example we explore a situation of this type, and consider the frictionless and elastic impact between a deformable sphere and an elastic plate. The simulation illustrates the ability of the method to model a very large number of impacting "particles", allowing a very robust simulation of fragmentation.

The sphere of the example has a radius of $25 \mathrm{~mm}$, it is launched towards the plate with a velocity of $300 \mathrm{~m} / \mathrm{s}$ in the normal direction, and is discretized with a meshfree method, employing 189 nodes. For high velocity impacts such as the one under consideration, it is often decided that the impactor behaves like a compressible fluid, breaking for any negative pressure. In this simulation, the sphere is modeled with a fluid of dynamic viscosity $\nu=0.1 \mathrm{~Pa} \cdot \mathrm{s}$, density $\rho=3000 \mathrm{~kg} / \mathrm{m}^{3}$, and a linear equation of state with bulk modulus $K=5 \mathrm{GPa}$. The plate has dimensions $0.01 \times$ $0.15 \times 0.15 \mathrm{~m}^{3}$ and is discretized with a finite element model of 512 nodes, with all the 


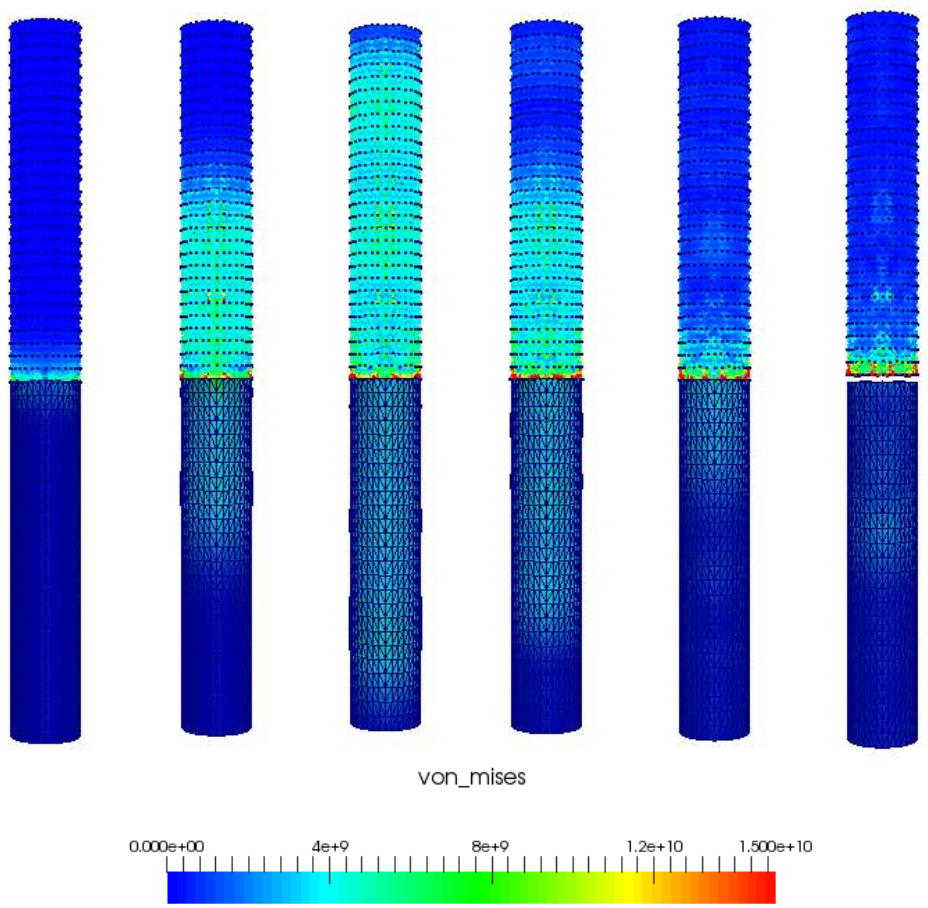

FIGURE 3.8: Double Taylor bar impact, Von Mises stress (Pa). From left to right, solutions at time $t=1,5,9,12,16,21 \mu \mathrm{s}$.

displacements of the lateral surfaces constrained. The material of the plate is neohookean, with Young's modulus $E=1 \mathrm{GPa}$, Poisson's ratio $\nu=0.33$, and density $\rho=1740 \mathrm{Kg} / \mathrm{m}^{3}$. The evolution of the von Mises stress is plotted in Fig. 3.9. where the fragmentation of the (meshfree) sphere can be observed. The vertical reaction of the plate supports, as a function of time, is depicted in Fig. 3.10. As a result of the vibrations induced on the plate, the maximum upwards reaction takes place at approximately $150 \mu \mathrm{s}$, followed by a reversal of the sign of this force. During the impact, the sphere breaks into many small pieces, and each of them continues to interact with the plate. The contact algorithm, without any additional modification, deals with the new surface interactions.

\section{Sliding block on an inclined plane}

Now we perform the sliding of a deformable neo-hookean cube of side length $0.03 \mathrm{~m}$ with Young's modulus of value $70 \mathrm{GPa}$ and Poisson's ratio 0.33 , along a rigid plane with an inclination of $\alpha=15^{\circ}$. This test is performed to assess the performance of the contact algorithm with friction. See Fig. 3.11 for an illustration. The contact considered is completely elastic $(e=1)$ and with a friction coefficient $\mu=0.1$. The density of the sliding box is $2800 \mathrm{~kg} / \mathrm{m}^{3}$ and gravity has been set to the value $g=2000 \mathrm{~m} / \mathrm{s}^{2}$ to reduce the simulation time. The cube has been discretized with a meshfree method, employing 125 nodes, while the motion of the plane is completely constrained. The analytical solution for the acceleration of a sliding cube is

$$
a_{\text {plane }}=g(\sin (\alpha)-\mu \cos (\alpha)) .
$$

Since the acceleration is constant, the kinetic energy of the cube, given its total mass $m$, is

$$
K=\frac{1}{2} m\left(a_{\text {plane }} t\right)^{2} .
$$




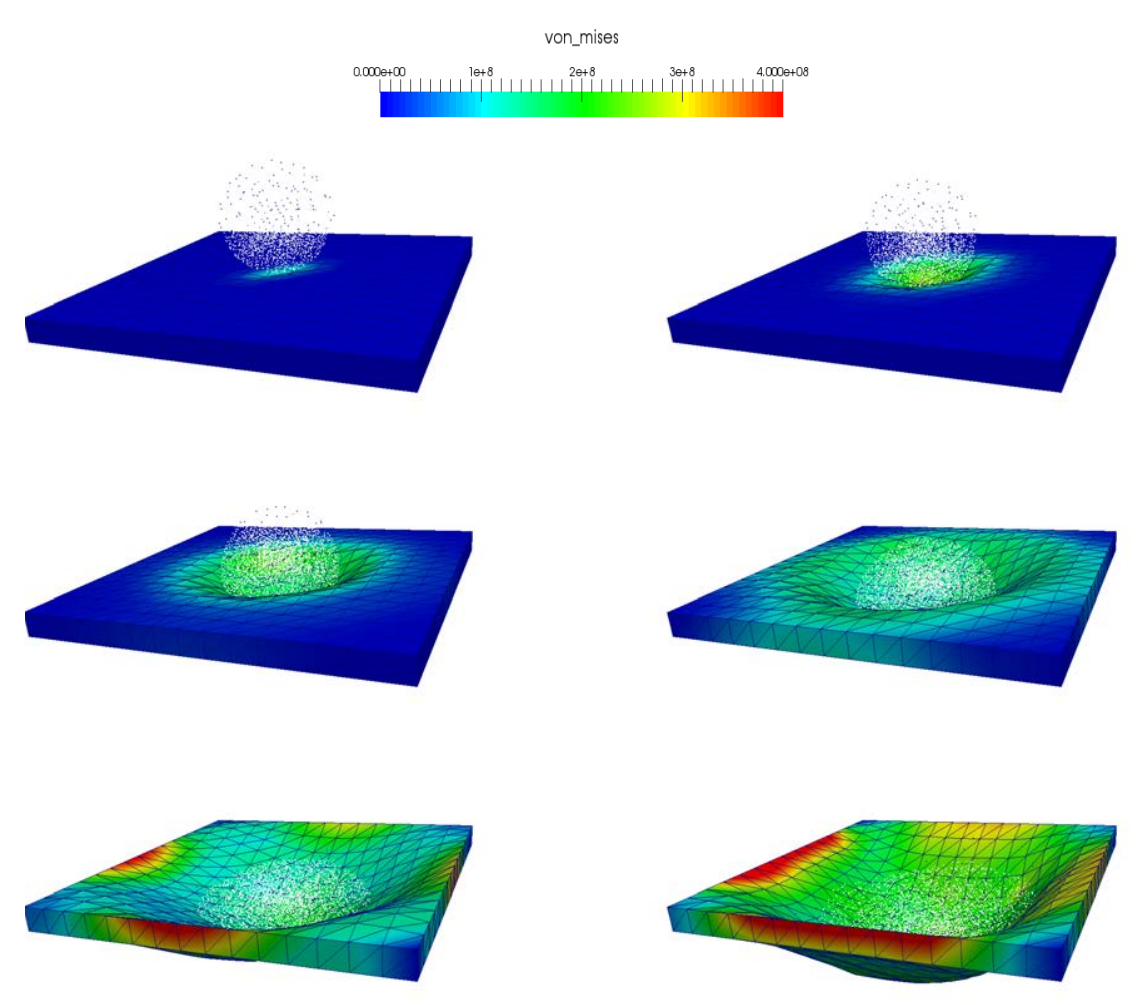

FIGURE 3.9: Von Mises stress (Pa) of the sphere impact onto deformable plate. From left to right, top to bottom, solutions at time $t=5,32,80,132,200,302 \mu \mathrm{s}$.

This solution is compared in Fig. 3.12 with the kinetic energy in the simulation, and proves that the frictional contact algorithm captures accurately the global motion of the sliding block.

\section{Multi-body impact}

A multi-body simulation is performed with elastic contact among deformable bodies. All of the latter are cubes of volume $0.03^{3} \mathrm{~m}^{3}$, two of them discretized with a meshfree method, and another three with finite element meshes. The cubes are set at the beginning of the simulation with their edges aligned with the coordinate axes. The meshfree bodies have their centers of mass at the points $(0,0,0) \mathrm{m}$ and $(0,0.0315,0.0315) \mathrm{m}$. The centers of mass of the three finite element cubes are, respectively, placed initially at the points $(0,0.0315,0.063) \mathrm{m},(0,-0.0315,0) \mathrm{m}$ and $(0,0.0315,0) \mathrm{m}$ (see Fig. 3.13 for an illustration). All the bodies employ the same neo-hookean material with Young's modulus $71 \mathrm{GPa}$, Poisson's ratio 0.33 and density $2800 \mathrm{~kg} / \mathrm{m}^{3}$. The finite element and meshfree cubes are discretized with 125 nodes. An initial velocity $(25.0,-120.0,100.0) \mathrm{m} / \mathrm{s}$ is applied to the cube whose center of mass has position $(0,0.0315,0)$, setting up its motion and, due to the contacting forces, the motion of all remaining cubes.

Fig. 3.14 shows the evolution of the linear momentum, which remains constant at all times. This is due to the fact that the standard central difference method preserves the linear momentum, and that the contact algorithm is designed to preserve it during the projection step. Fig. 3.15 depicts the energy evolution, whose oscillations can be attributed to both the central difference scheme and the contact interactions. 


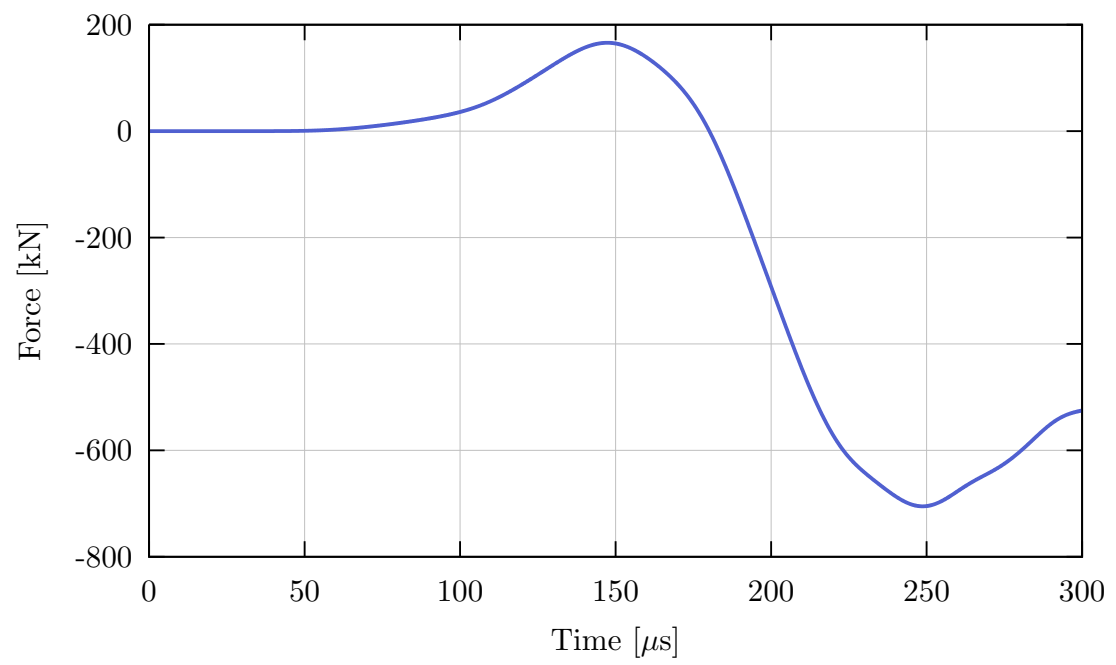

FIGURE 3.10: Normal force exerted on the plate.

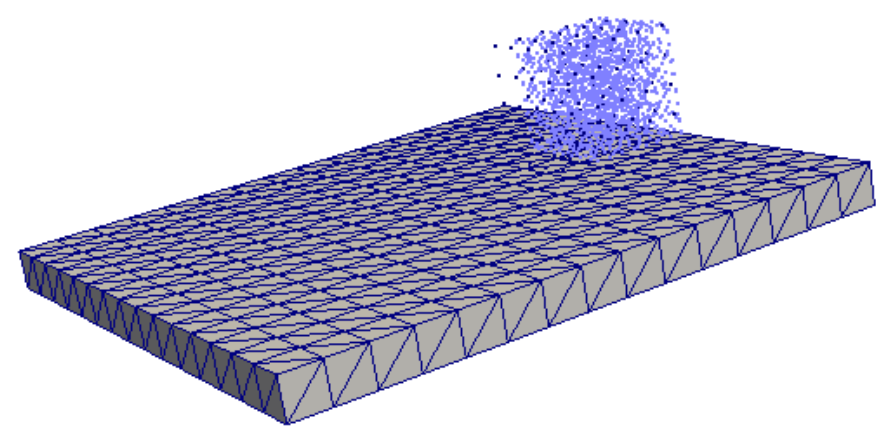

FIGURE 3.11: Meshfree cube on inclined plane.

Finally, the evolution of von Mises stress on the cubes is shown in Fig. 3.16, where the large motions of all the bodies can be observed.

\section{Taylor's bar impact}

We study next Taylor's high velocity anvil impact [104], an experiment for which there exists a wealth of experimental data and is often employed to study the rate dependent elastoplastic behavior of materials and the ability of algorithms to capture it [105], [106]. Taylor's test consists of a metallic cylinder launched at high velocity against a wall or plate. When one of the flat surfaces of the cylinder impacts, the specimen deforms plastically and its final shape - which conserves the axial symmetry - can be measured easily.

One of the constitutive laws that is often employed to model metals with rateand temperature-dependent elastoplastic behavior is Johnson-Cook's [107]. This $J_{2}$ 


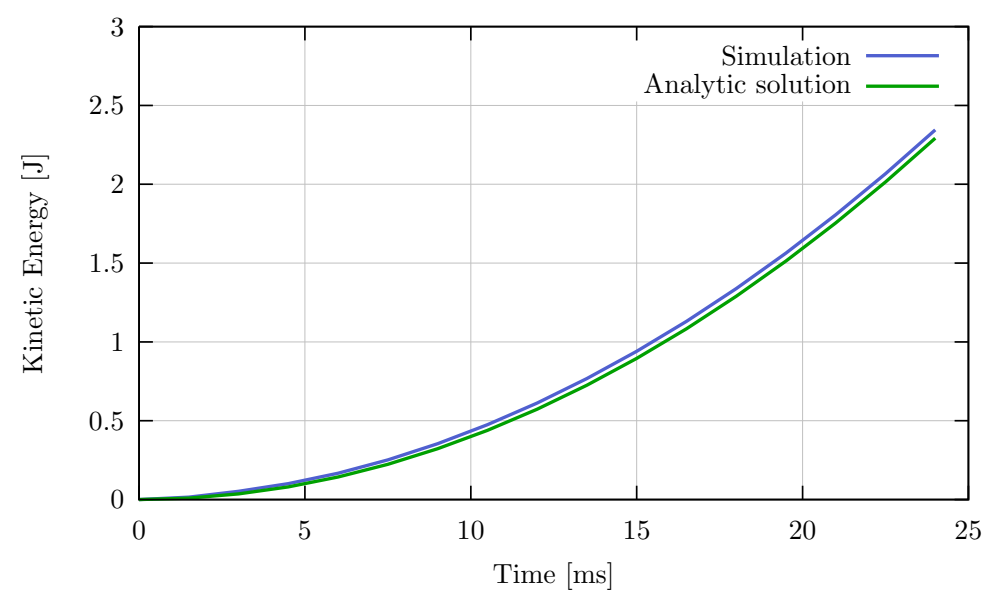

FIGURE 3.12: Kinetic energy of the sliding cube on an inclined plane.

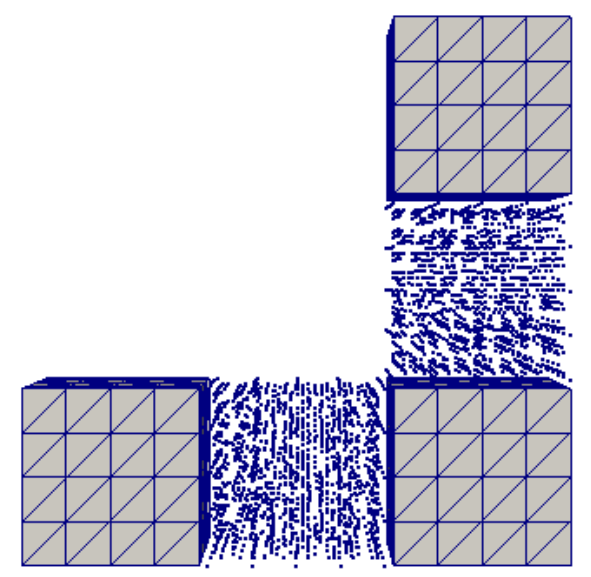

FIGURE 3.13: Arrangement of finite element / meshfree cubes.

model employs a yield stress of the form

$$
\sigma_{y}=\left(A+B \epsilon^{N}\right)\left(1+C \log \frac{\dot{\epsilon}}{\epsilon_{0}}\right)\left(1-\left(\frac{T-T_{0}}{T_{m}-T_{0}}\right)^{M}\right) .
$$

In this expression, $\epsilon$ is the accumulated plastic strain, $\dot{\epsilon}$ its rate, and $T, T_{m}$ and $T_{0}$ are, respectively, the current, melting, and reference temperatures of the material. The parameters $A, B, N, M, \dot{\epsilon}_{0}$ are material constants that, together with Lamé parameters $\lambda$ and $\mu$, completely determine the response of the material.

$$
\begin{array}{ccccccc}
A & B & C & N & M & T_{0} & T_{m} \\
\mathrm{MPa} & \mathrm{MPa} & - & - & - & \mathrm{K} & \mathrm{K} \\
\hline 90 & 292 & 0.025 & 0.31 & 1.09 & 300.0 & 1356.0
\end{array}
$$

TABLE 3.1: Johnson-Cook constants for copper.

Based on this test, we analyze next the impact of a copper cylinder launched onto a wall at $227 \mathrm{~m} / \mathrm{s}$ [105], [107], [108]. The parameters of the Johnson-Cook's model are given in Table 3.1. The density is taken to be $\rho=8960 \mathrm{~kg} / \mathrm{m}^{3}$ and the Lamé elastic constants are $\lambda=150 \times 10^{3} \mathrm{MPa}, \mu=75 \times 10^{3} \mathrm{MPa}$. The simulation assumes 


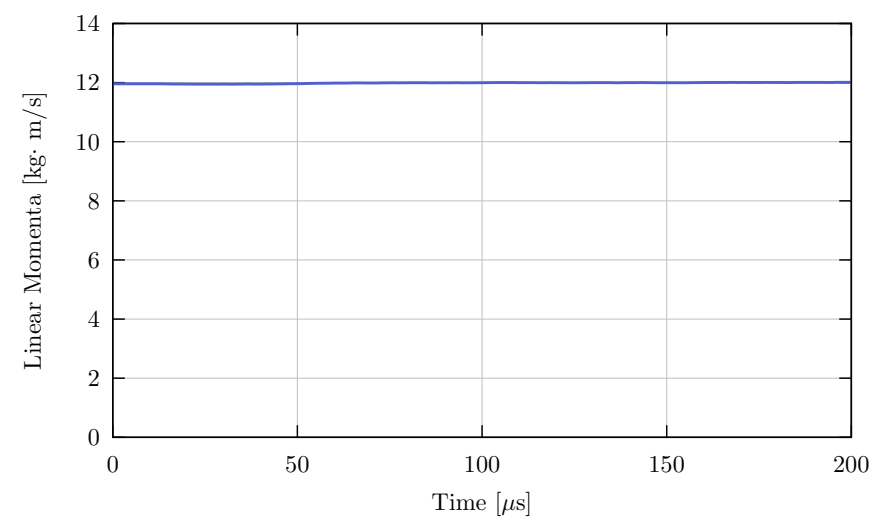

FIGURE 3.14: Linear momenta evolution for multi-body impact.

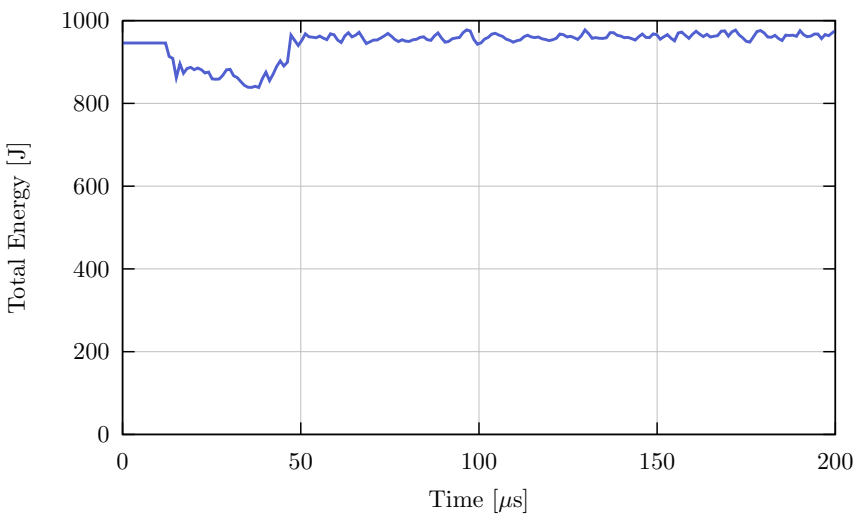

FIGURE 3.15: Total energy evolution for multi-body impact.

isothermal conditions at temperature $T=300 \mathrm{~K}$ and the anvil initial diameter and length are, respectively, $7.62 \mathrm{~mm}$ and $25.4 \mathrm{~mm}$.

In the simulation, the cylinder is placed $1.3 \mathrm{~mm}$ away from the plate, which is considered to be completely rigid by constraining the displacements of all of its nodes. The Coulomb friction coefficient in the contact is assumed to be constant and equal to 0.1 , and the restitution coefficient is taken to be zero, modeling a perfectly inelastic impact. Approximately $5.7 \mu$ s after the anvil is launched, it impacts the plate and starts deforming with large plastic deformations. Figure 3.17 shows six snapshots of the simulations, illustrating the deformations in the model and the field of plastic slip. The figures depict this slip at the material points, and also shows in black the nodes in the finite element and meshfree bodies.

\subsubsection{Oblique elastoplastic impact}

In the last example, we consider the high-velocity, elasto-plastic oblique impact with friction of a deformable sphere against a deformable plate as previously proposed in the literature [109]. The diameter of the sphere and the thickness of the plate are both equal to $6.4 \mathrm{~mm}$. The other two dimensions of the plate are taken to be equal to $20 \mathrm{~mm}$, in $x$ direction, and $30 \mathrm{~mm}$ in $y$ direction. See Fig. 3.18 for an illustration of the geometry and the definition of the coordinate axes. The sphere has an initial velocity of $700 \mathrm{~m} / \mathrm{s}$ towards the plate with an angle of 0.35 rad with respect to the plate's surface. 
The sphere and plate are both of steel. The former has the properties of S7 steel, whereas the latter is of type 4340. Both materials are modeled with a JohnsonCook's law (see Eq. 3.83) with the data given for these two steels in their original article [107]. For convenience, these data are summarized in Table 3.2, including the density $\rho$. In addition, for both materials we have considered that Young's modulus is $210 \mathrm{GPa}$, Poisson's ratio is 0.3 , and assumed isothermal conditions at $300 \mathrm{~K}$.

\begin{tabular}{ccccccccc} 
Steel type & $\begin{array}{c}A \\
\mathrm{MPa}\end{array}$ & $\begin{array}{c}B \\
\mathrm{MPa}\end{array}$ & $\begin{array}{c}C \\
\end{array}$ & $\begin{array}{c}N \\
-\end{array}$ & $\begin{array}{c}M \\
-\end{array}$ & $\begin{array}{c}T_{0} \\
\mathrm{~K}\end{array}$ & $\begin{array}{c}T_{m} \\
\mathrm{~K}\end{array}$ & $\begin{array}{c}\rho \\
\mathrm{kg} / \mathrm{m}^{3}\end{array}$ \\
\hline 4340 & 792 & 510 & 0.014 & 0.26 & 1.03 & 300 & 1793 & 7830 \\
$\mathrm{~S} 7$ & 1539 & 447 & 0.012 & 0.18 & 1.00 & 300 & 1763 & 7750
\end{tabular}

TABLE 3.2: Johnson-Cook constants for steels.

The meshfree sphere includes 189 nodes and 3072 material points. The finite element model of the plate has approximately 37000 elements and 7500 nodes. All the nodes on the lateral faces of the plate have their degrees of freedom constrained. The contact is completely elastic and Coulomb's friction coefficient is 0.05. Figure 3.19 shows six snapshots of the impact process, cutting the model by the symmetry plane perpendicular to the $x$ axis. The figure illustrates the presence of plastic deformation in the finite element and meshfree bodies.

The evolution of the linear momentum and energy of the sphere are shown in Figs. 3.20 and 3.21 , respectively. The first figure confirms a smooth change of momentum in both the normal and tangential directions to the plate. The second one illustrates the good energy control of the method during the contact phase. We note that no smoothing has been applied on any of the curves depicted.

\subsection{Concluding remarks}

In this chapter we have analysed the available meshfree/particle discretisation methods, discarding SPH method since it has undesirable instabilities. We concluded that those who preserve the Kronecker-delta property are the best choice for explicit simulations of contact since the enforcement of impenetrability and the update of velocities when using a predictor-corrector contact algorithm can be done in a straightforward manner.

We have also presented a new algorithm for contact/impact in explicit simulations of deformable bodies. The proposed method has several salient features that make it very appealing for general purpose computations. First, it imposes very mild stability restrictions on the time step size employed in the integration. Second, it bypasses the need to choose a penalty parameter since the impenetrability constraint is not imposed with an artificial stiffness, but rather with a projection scheme. Third, it possesses favorable energy estimates for contact with or without friction and/or perfect restitution. Fourth, it is asymmetric by construction, and thus can be employed for modeling interactions between finite element models, or between a finite element model and a meshfree one.

Overall, the method is simple to implement, and extremely robust for all types of contact simulations, including those involving bodies with sharp edges or corners. We have illustrated its performance by means of representative examples, including high velocity impacts, contact in fragmenting bodies, and impact among bodies with sharp corners, all of them involving both finite element and meshfree discretisations. 

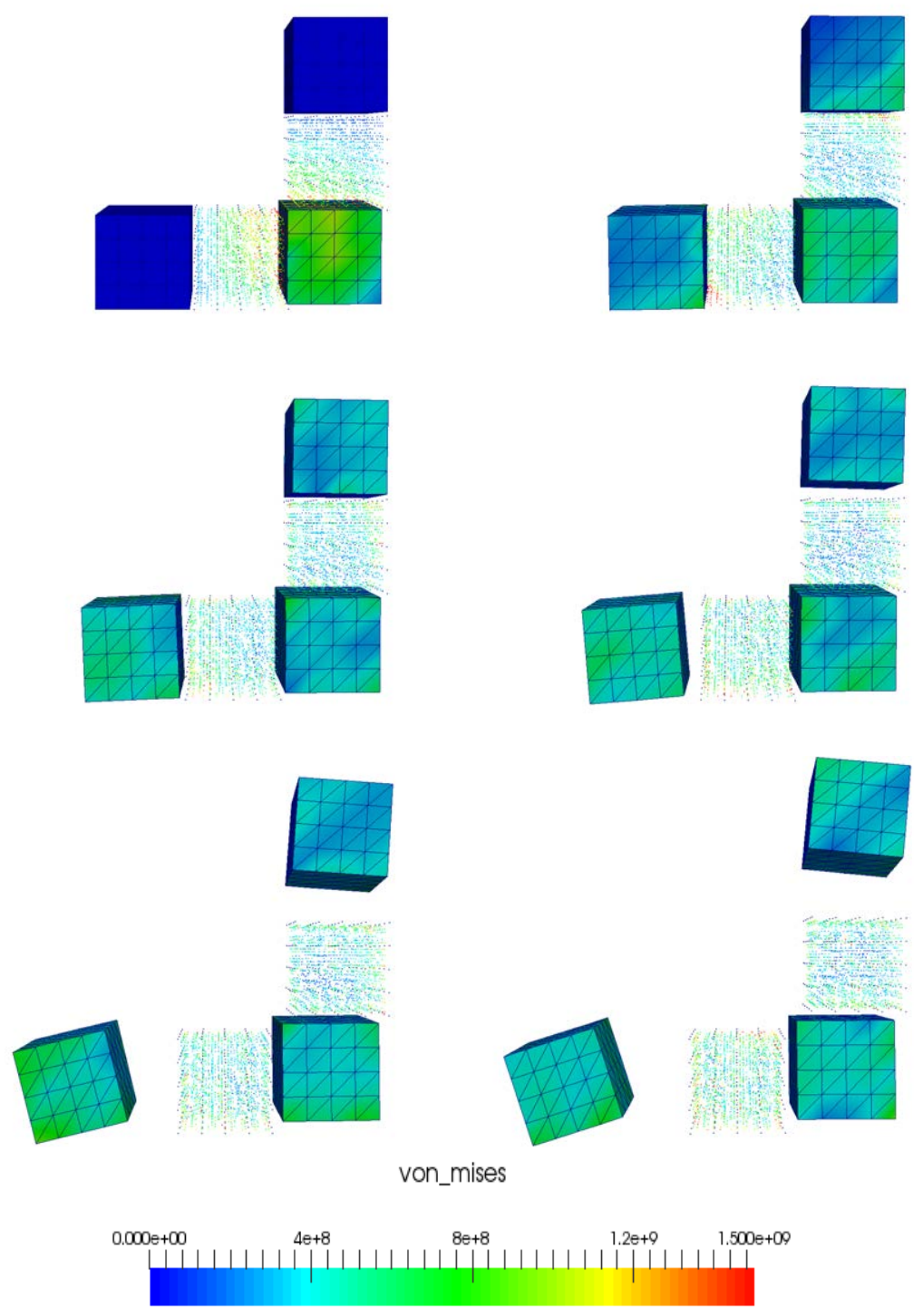

FIGURE 3.16: Von Mises stress (Pa) for multi-body impact. From left to right, top to bottom, solutions at time $t=19,49,79,128,295,376$ $\mu \mathrm{s}$. 

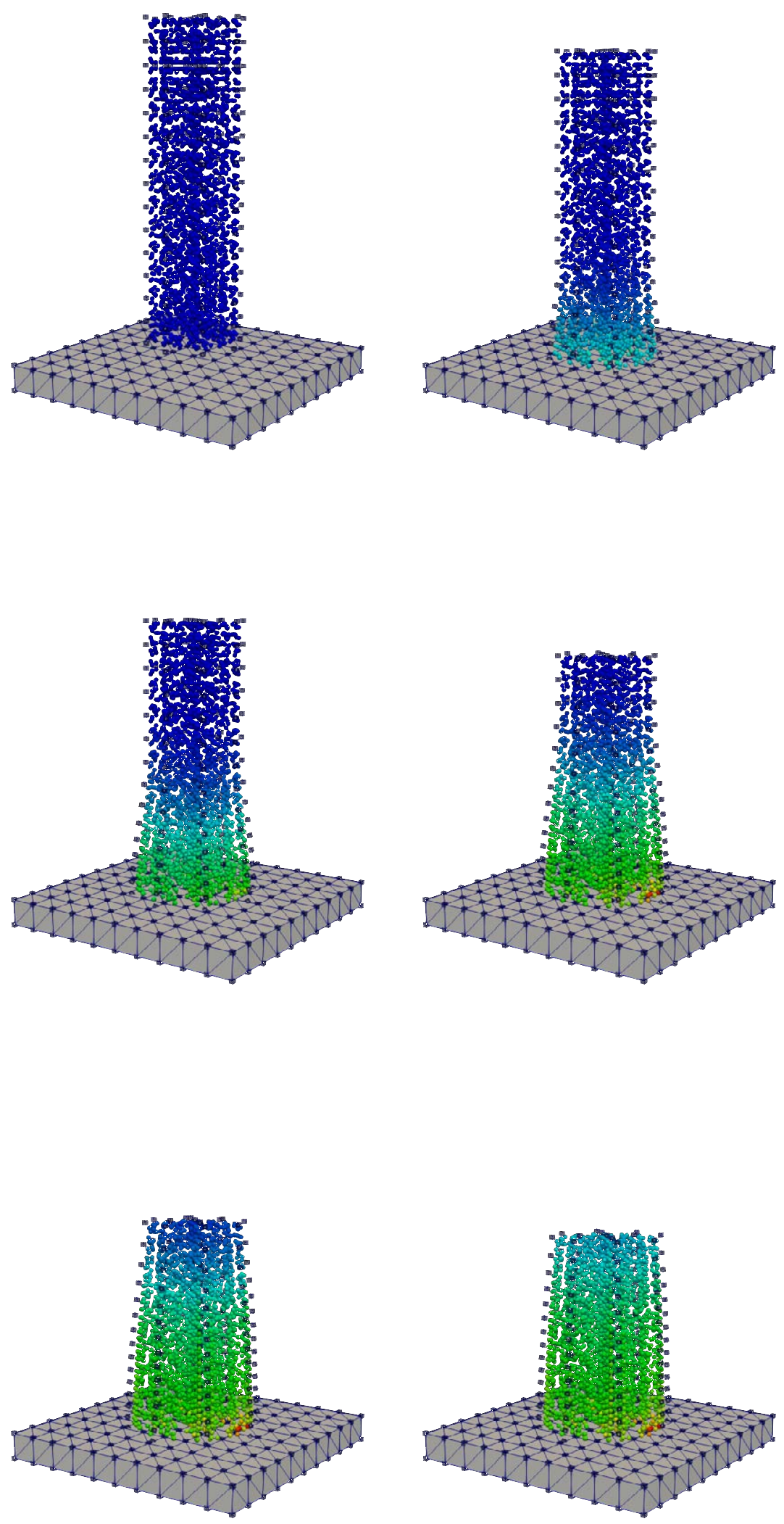

Plastic slip

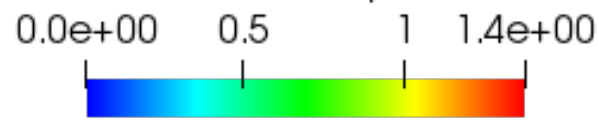

FIGURE 3.17: Taylor's anvil test. From left to right, top to bottom, deformed configurations at time $t \sim 11.2 k \quad \mu \mathrm{s}$, with $k=0,1, \ldots, 5$, showing the plastic slip evolution. 


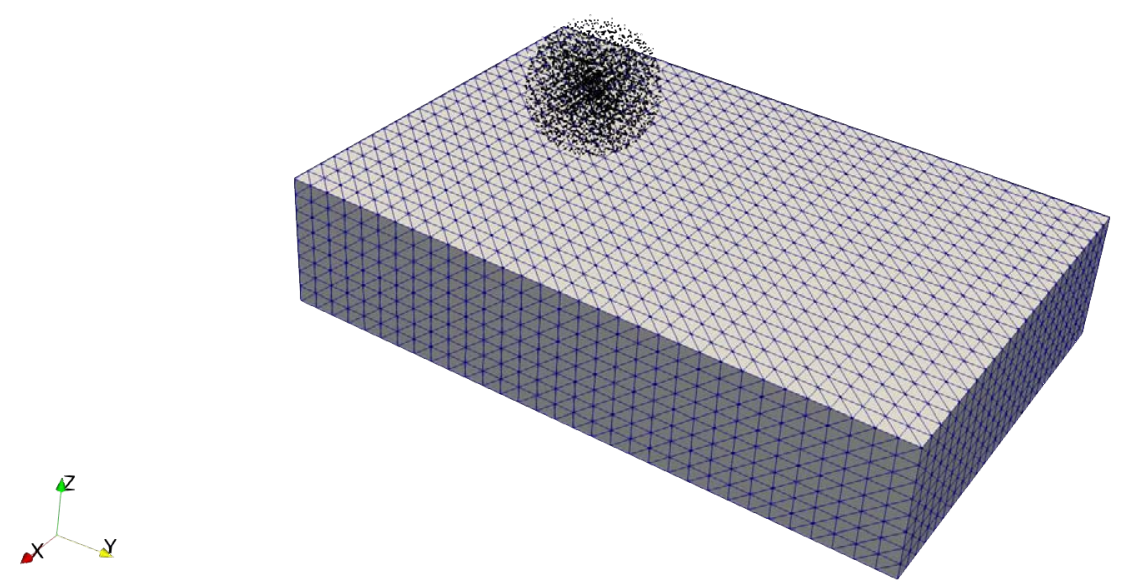

FIGURE 3.18: Elastoplastic of a sphere on plate. Initial configuration, showing the finite element mesh and meshless body.
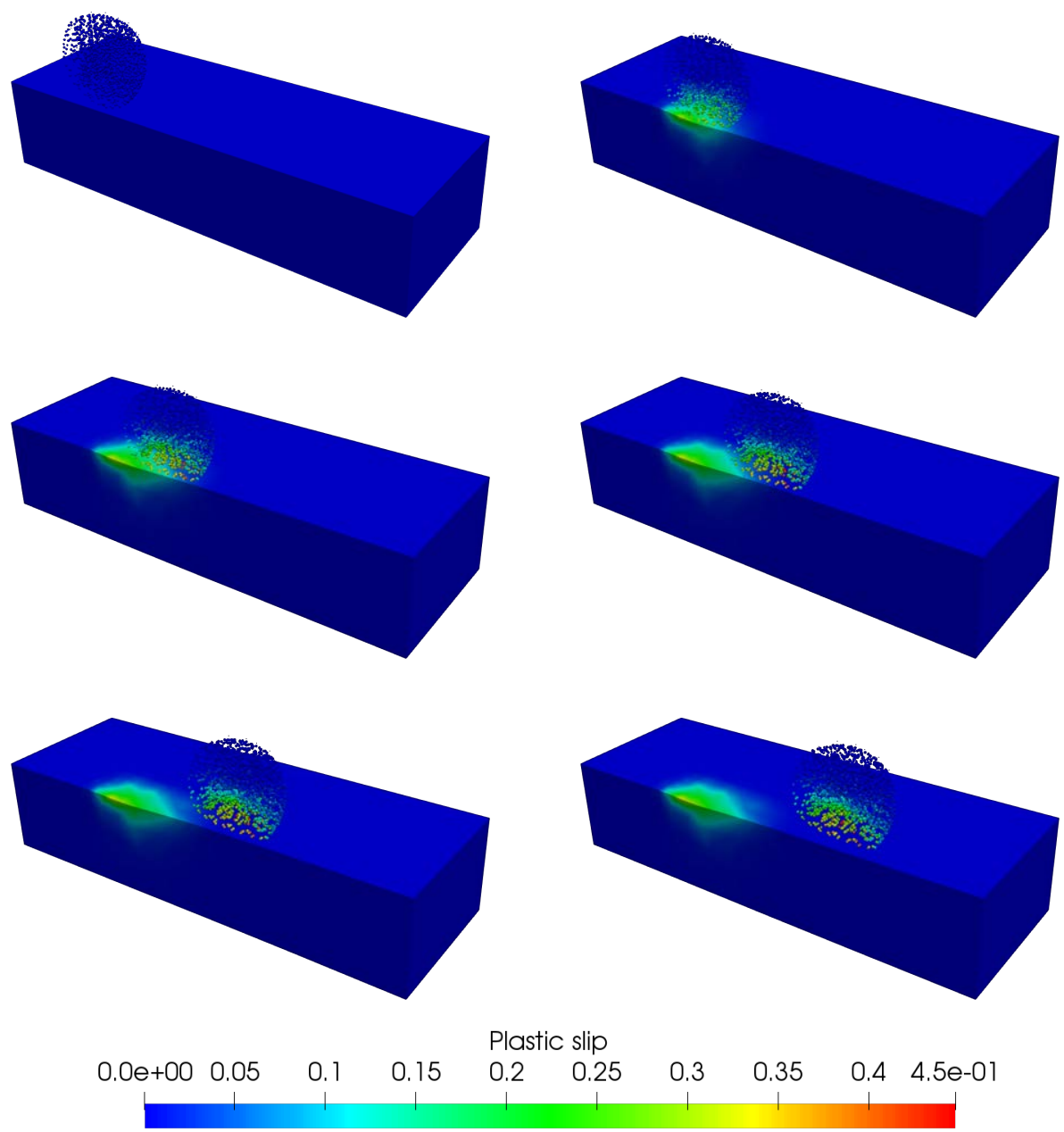

FIGURE 3.19: Impact of elastoplastic sphere. From left to right, top to bottom, deformed configurations at time $t \sim 4 \cdot k \mu \mathrm{s}$, with $k=$ $0,1, \ldots, 5$, showing the plastic slip evolution and cut by the plane of symmetry. 


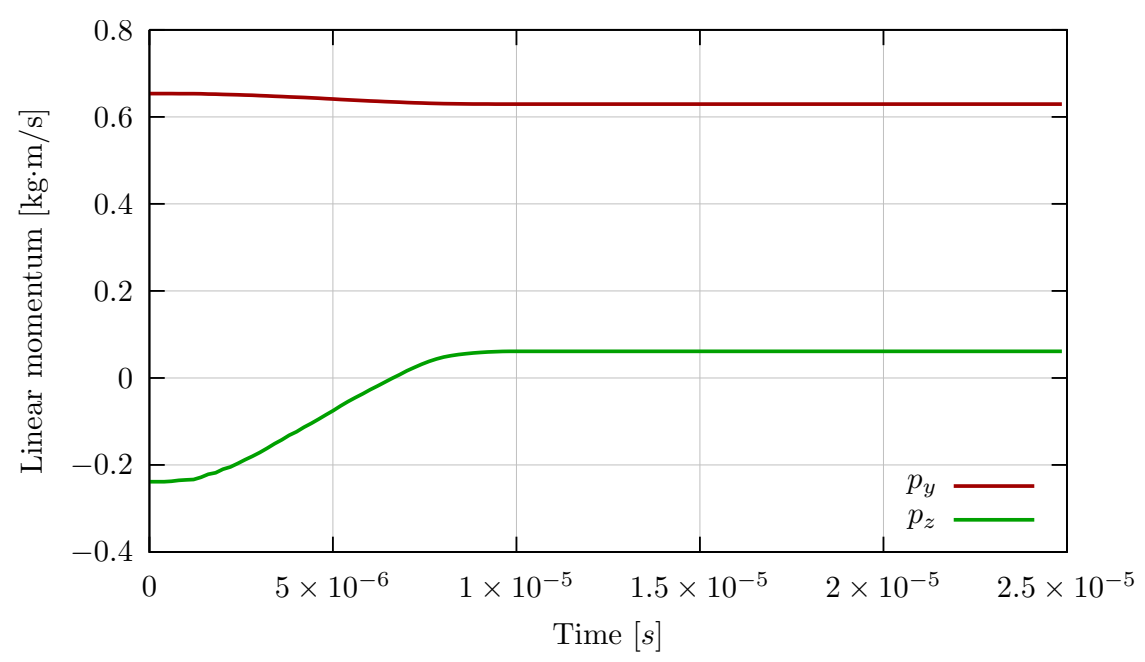

FIGURE 3.20: Linear momentum components of the sphere during the impact of example 3.4 .9

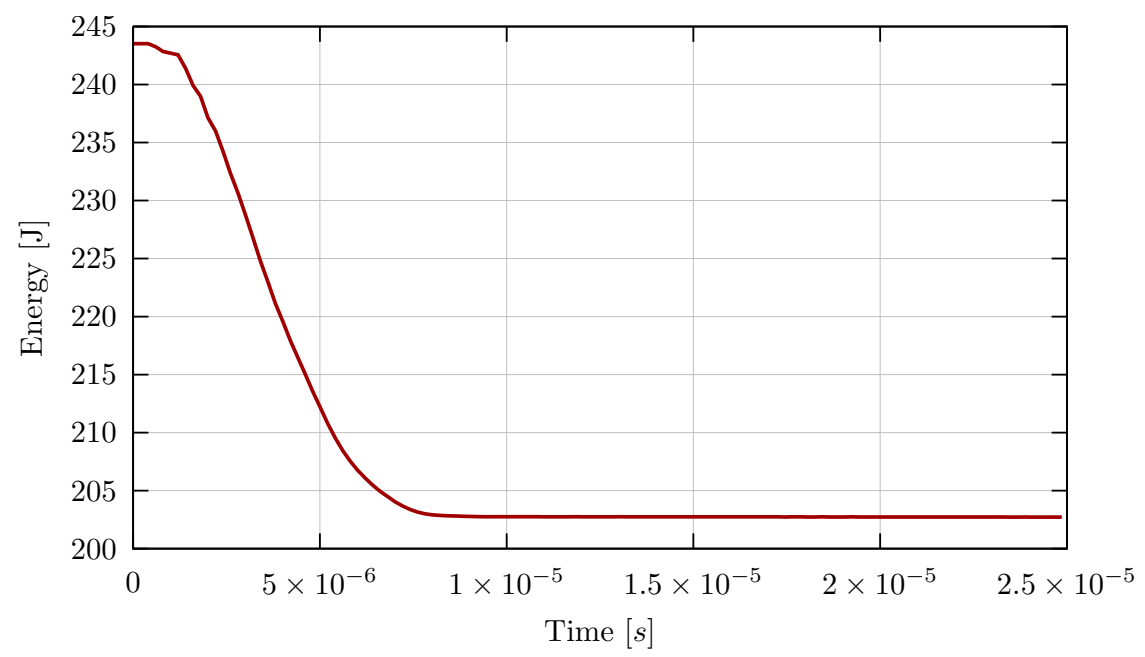

FIGURE 3.21: Total energy of the sphere during the impact of example 3.4 .9 


\section{Chapter 4}

\section{Simulations}

\subsection{Ice impact onto leading edge}

As a conclusion of the tools developed in this work, we perform a more complex simulation of the impact of an ice ball onto a leading edge, see Fig. 4.1.

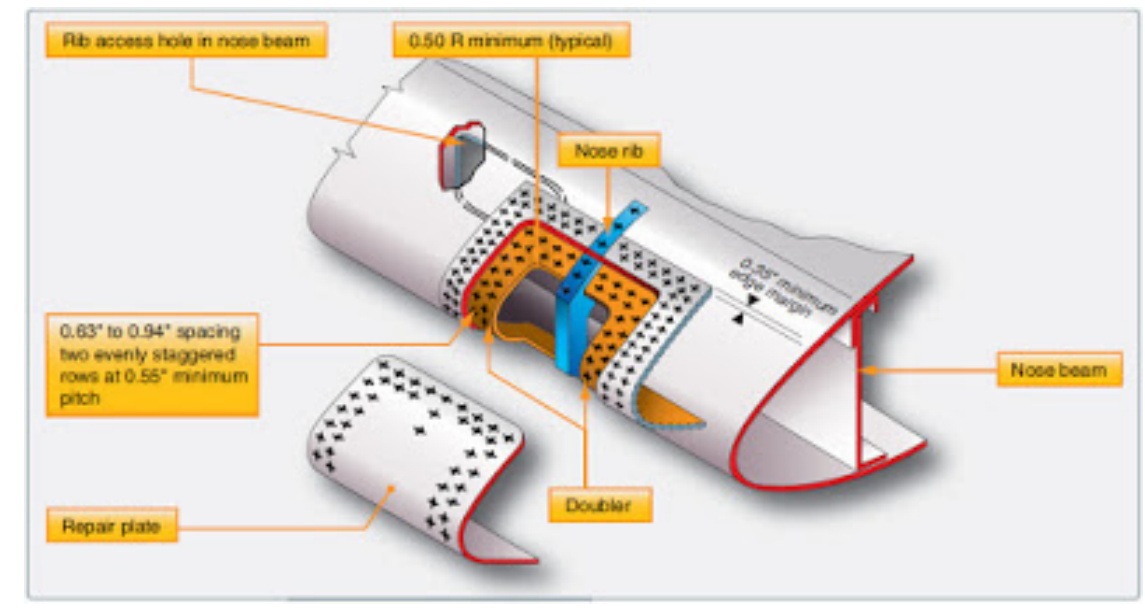

FIGURE 4.1: Leading edge.

We will measure the damage from both a qualitative and quantitative perspective and its sensibility to different impact conditions, i.e. different ball sizes, different impact positions, different velocities of the impacting ice sphere and different strength thresholds for the ice sphere. The aim of this set of simulations is to give an estimation of the possible damage and its magnitude due to FOD under different conditions. The quantification of the damage is obtained through the integration of the so-called plastic slip in $\mathcal{B}_{i}$, where $\mathcal{B}_{i}$ in this case is the body that discretises the leading edge. Thus this positive scalar representing damage is expressed as:

$$
\text { plastic damage }:=\int_{\mathcal{B}_{i}} \varepsilon^{p}: \varepsilon^{p} \mathrm{~d} V
$$

The leading edge is discretised with 7005 nodes and is represented with a parabolic shape, with a thickness of $0.425 \mathrm{~cm}$. The two other dimensions are depicted in Fig. 4.2 and Fig. 4.3 . Since the leading edge is attached to the stinger of the wing as can be seen in Fig. 4.1 we will constraint in directions $\mathrm{x}, \mathrm{y}$ and $\mathrm{z}$ the faces depicted in Fig. 4.3. 


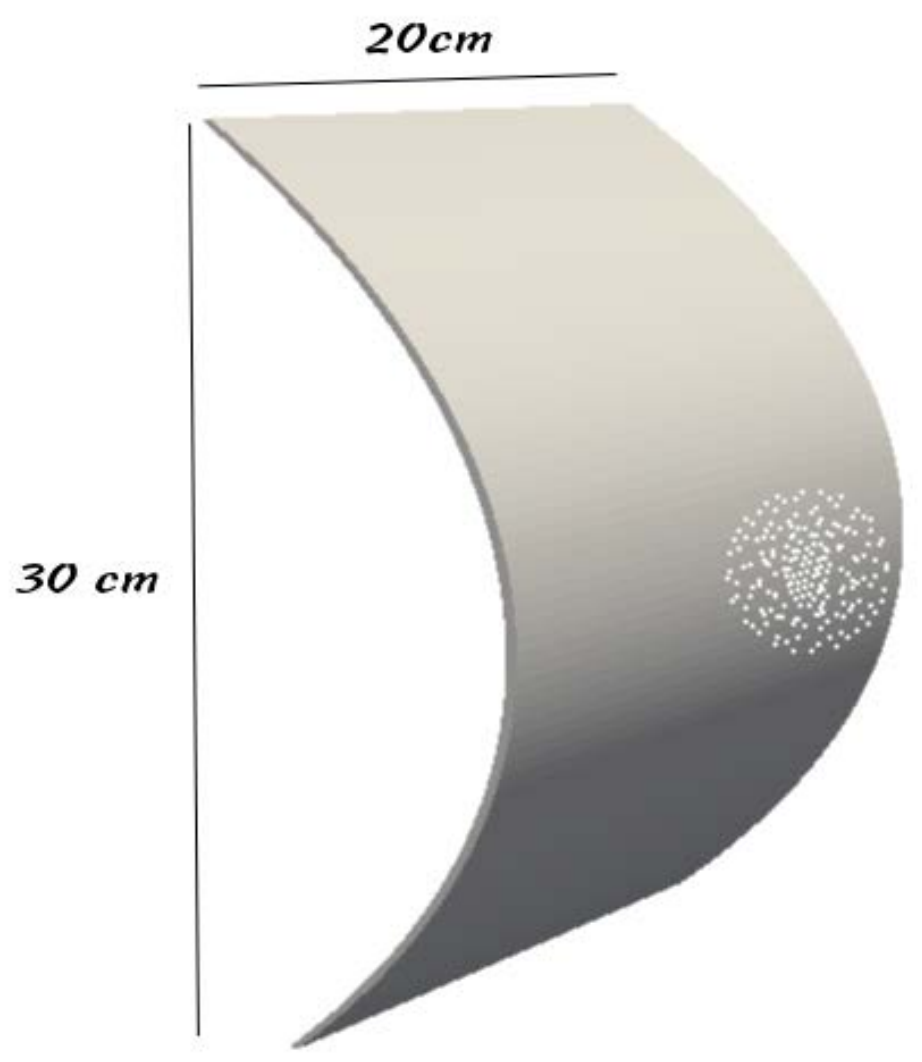

FIGURE 4.2: Discretised leading edge.

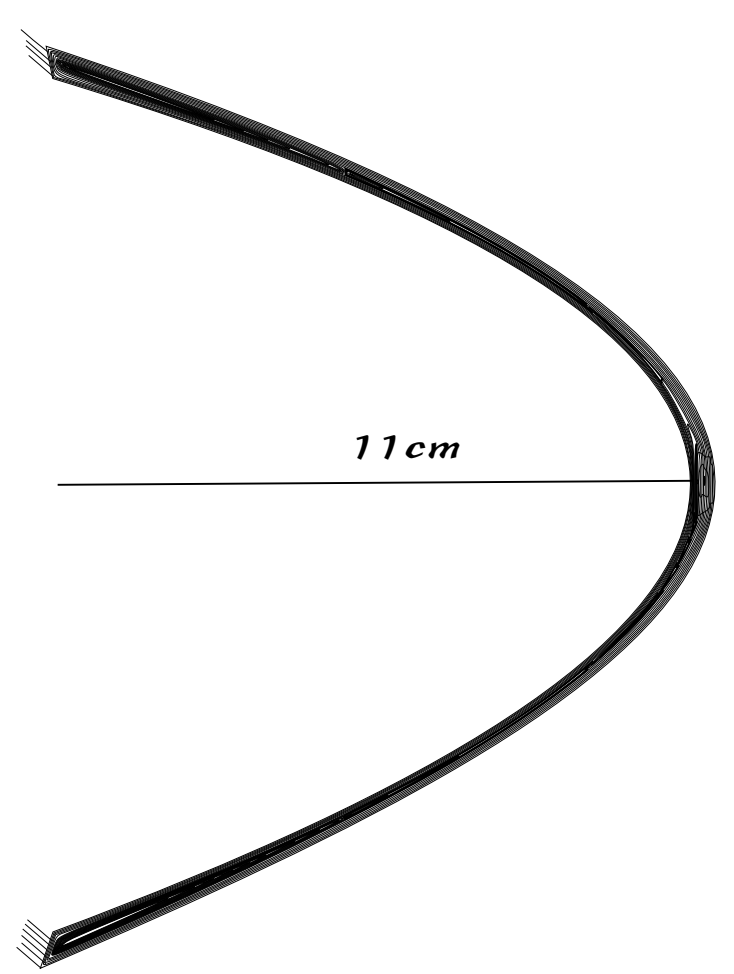

FIGURE 4.3: Discretised leading edge. 
We will make use of the viscoplasticity developed in Chapter 2. We will use for both bodies, the leading edge and the ice sphere a potential law for the rate effects with $n=2$. The material of the leading edge is intended to represent aluminium. With the theory developed in Chapter 2 we will use a viscoplastic model using a logarithmic measure based on the Tresca plasticity criteria where yield happens due to deviatoric stress, which is a faithful approach for metals. The ice is represented with

\begin{tabular}{|c|c|c|c|c|c|}
\hline$E$ & $\nu$ & $\rho$ & $H_{\text {iso }}$ & $\sigma_{0}$ & $\tau$ \\
\hline $70 \mathrm{GPa}$ & 0.33 & $2700 \mathrm{~kg} / \mathrm{m}^{3}$ & $10 \mathrm{MPa}$ & $150 \mathrm{MPa}$ & $90 \mathrm{~ms}$ \\
\hline
\end{tabular}

TABLE 4.1: Tresca viscoplastic model for leading edge.

a discretised sphere of 189 nodes and with a Drucker-Prager viscoplastic material using a logarithmic strain measure to account for large strain theory. As mentioned in Section 1.1.1 ice is rate and pressure dependent, thus a Drucker-Prager viscoplastic model fulfils this behaviour because is a plasticity criteria that depends on the volumetric and deviatoric stress part, pressure dependent, and the rate effects are accounted for with the viscous behavior. Finally to account for the brittle behavior

\begin{tabular}{|c|c|c|c|c|c|}
\hline$E$ & $\nu$ & $\rho$ & $\alpha$ & $\sigma_{0}$ & $\tau$ \\
\hline $10 \mathrm{GPa}$ & 0.33 & $1220 \mathrm{~kg} / \mathrm{m}^{3}$ & 0.15 & $826 \mathrm{kPa}$ & $50 \mathrm{~ms}$ \\
\hline
\end{tabular}

TABLE 4.2: Drucker-Prager viscoplastic model for ice sphere.

of ice and being able to obtain a crushed ice after impact, we add a failure criteria that is based on pressure thresholds both positive and negative, $p^{+}$and $p^{-}$respectively. Thus once the quadrature point attains a stress state $\sigma$ with its volumetric part not contained within that range, the quadrature point is broken, Therefore the condition for not broken can be expressed as

$$
p^{-} \leq \frac{-\operatorname{tr}[\boldsymbol{\sigma}]}{3} \leq p^{+} .
$$

To have a fluid-like behaviour at the broken state, for the subsequent stress states of that quadrature point, the deviatoric part of the stress state is removed and only positive pressures are allowed.

$$
\boldsymbol{\sigma}_{\text {broken }}= \begin{cases}\frac{\operatorname{tr}[\boldsymbol{\sigma}]}{3} \boldsymbol{I} & \text { if } \frac{-\operatorname{tr}[\boldsymbol{\sigma}]}{3} \geq 0, \\ \mathbf{0} & \text { if } \frac{-\operatorname{tr}[\boldsymbol{\sigma}]}{3}<0,\end{cases}
$$

with $I$ being the second order identity tensor. Now the different test conditions can be defined; we wil take as starting point the simulation called Reference 1 . This simulation is performed with the material parameters shown before, with a sphere of $5 \mathrm{~cm}$ in diameter aiming at the center of the leding edge and launched at $300 \mathrm{~m} / \mathrm{s}$ with $p^{+}$and $p^{-}$respectively $50 \mathrm{MPa}$ and $-50 \mathrm{MPa}$. The other simulations are defined from Reference 1 as follows:

- Reference 2, changes the ice sphere diameter from the size in Reference 1 to $10 \mathrm{~cm}$ in diameter.

- Reference 3, changes the ice sphere diameter from the size in Reference 1 to $2.5 \mathrm{~cm}$ in diameter.

- Reference 4, changes the ice sphere velocity from the speed in Reference 1 to $500 \mathrm{~m} / \mathrm{s}$. 
- Reference 5, changes the ice sphere velocity from the speed in Reference 1 to $100 \mathrm{~m} / \mathrm{s}$.

- Reference 6, changes the ice strength from Reference 1 to $p^{+}$and $p^{-}$respectively $100 \mathrm{MPa}$ and $-100 \mathrm{MPa}$.

- Reference 7, changes the ice strength from Reference 1 to $p^{+}$and $p^{-}$respectively $200 \mathrm{MPa}$ and -200 MPa.

- Reference 8, shifts the position of the ice sphere in Reference 1,7.5 cm in the perpendicular direction to the wing.

- Reference 9, shifts the position of the ice sphere in Reference 1, $11.25 \mathrm{~cm}$ in the perpendicular direction to the wing.

As one can infer, these 9 tests let us assess the influence in damage of the projectile speed, projectile strength, projectile impact position and finally the influence of the projectile mass by varying its size. Before going further in each analysis we show in Fig. 4.4 the evolution of the von Mises stress in Reference 1 in order to know the magnitude of loads during impact.

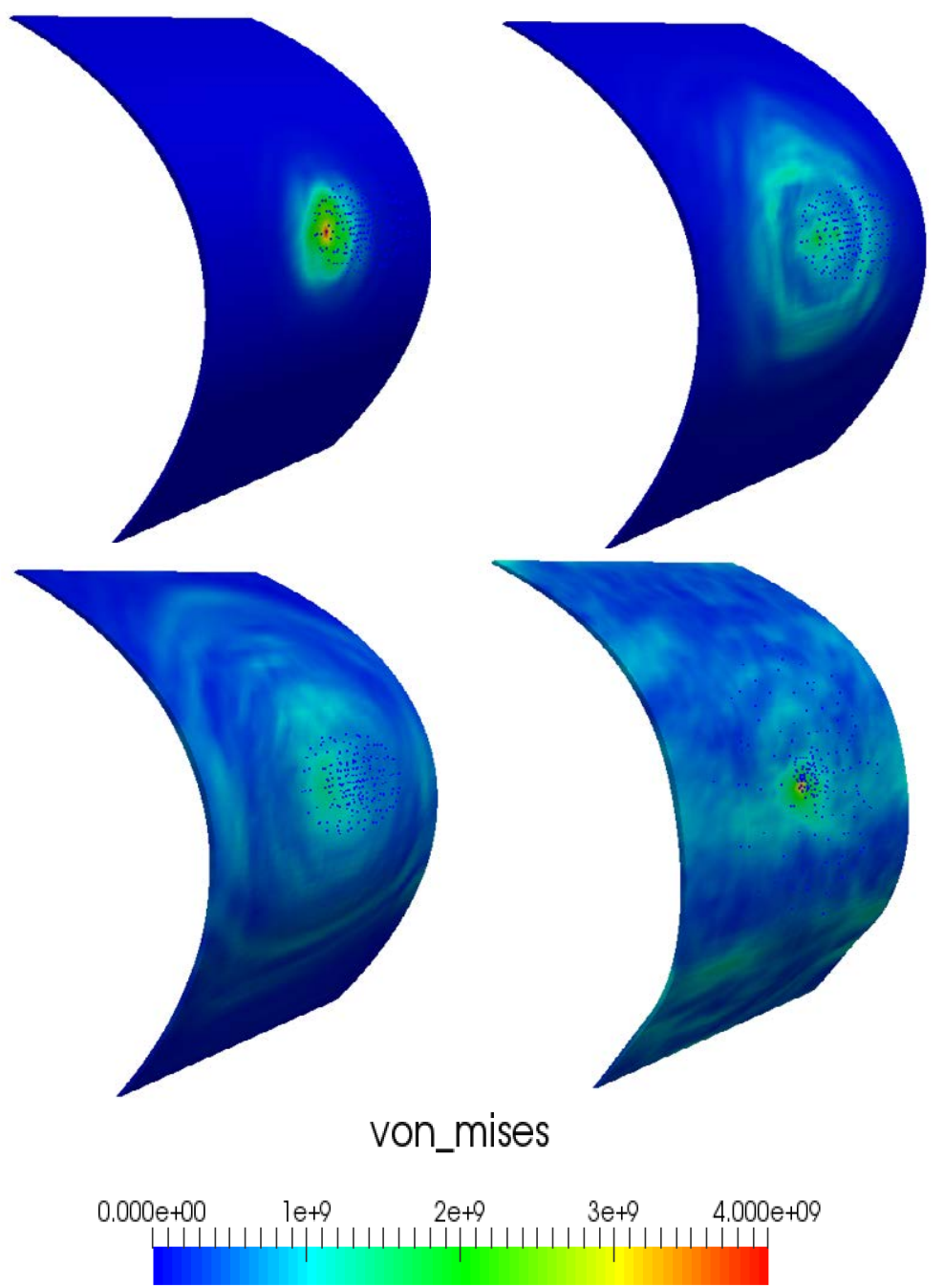

FIGURE 4.4: von Mises stress evolution in Reference 1 from left to right top to bottom at 36, 50, 75 and $200 \mu \mathrm{s},(\mathrm{Pa})$. 
The evolution of plastic slip is also shown in Fig. 4.5

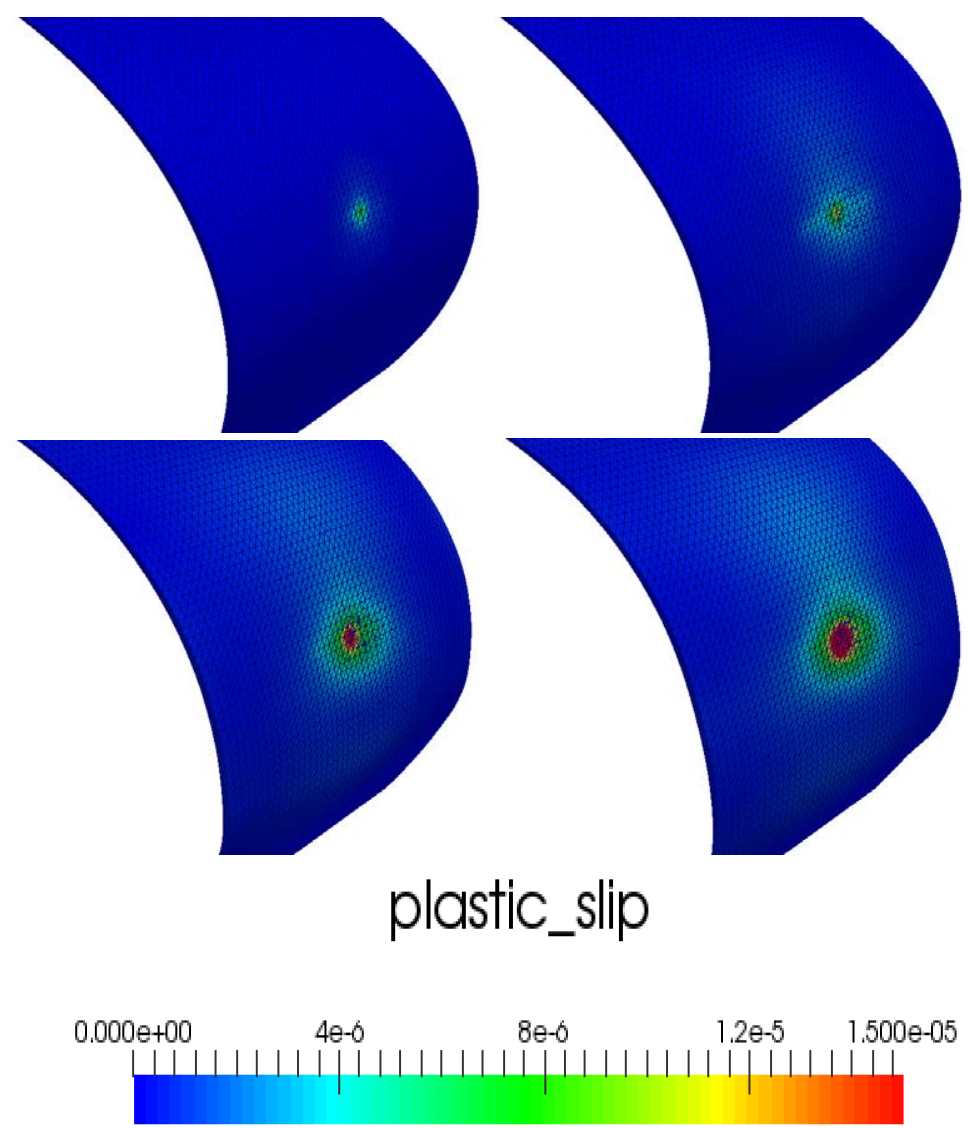

FIGURE 4.5: Plastic slip evolution in Reference 1 from left to right top to bottom at 40, 100, 160 and $200 \mu \mathrm{s}$.

The force exerted onto both constraints of the leading edge due to hailstone impact

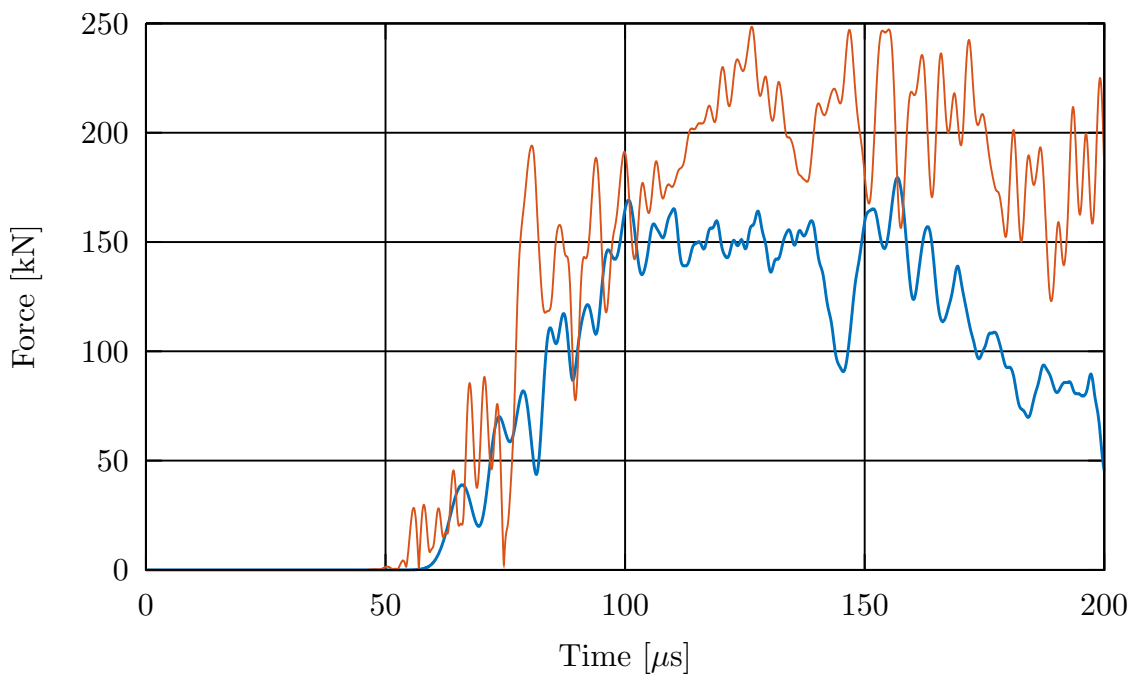

FIGURE 4.6: Force modulus exerted onto both constraints of the leading edge in Reference 1.

Fig. 4.7 shows the distortion of the mesh after failure of the ice sphere 


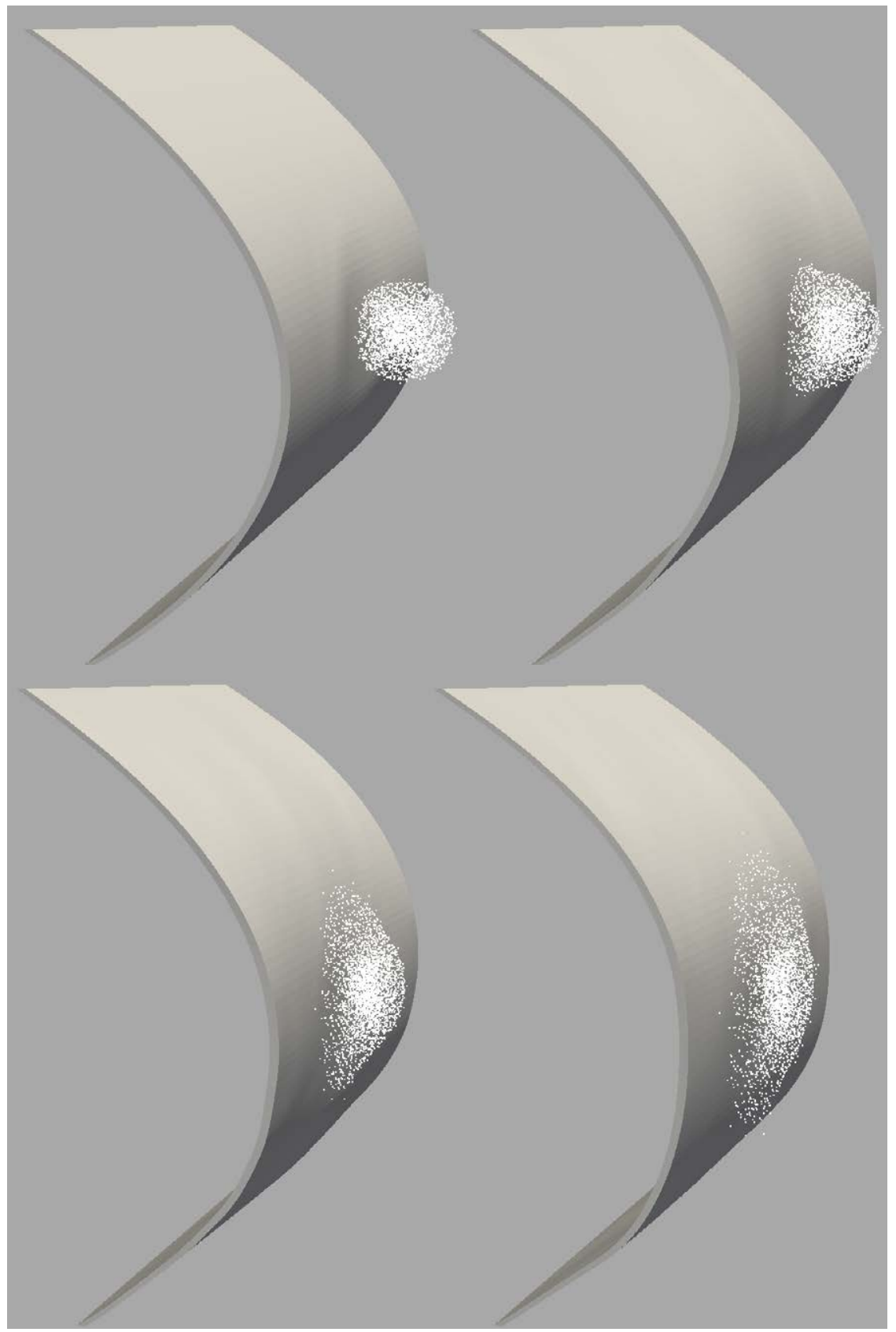

FIGURE 4.7: Ice mesh distortion evolution from left to right, top to bottom at 46, 90, 150 and $200 \mu$ s in Reference 1 .

Finally, in the paragraphs below we will analyse these 4 features from a qualitative and quantitative point of view.

\subsubsection{Projectile mass}

It is expected that an increase of mass will imply higher loads applied to the leading edge, since the inertia increases. These analysis includes Reference 1, Reference 2 and Reference 3. 


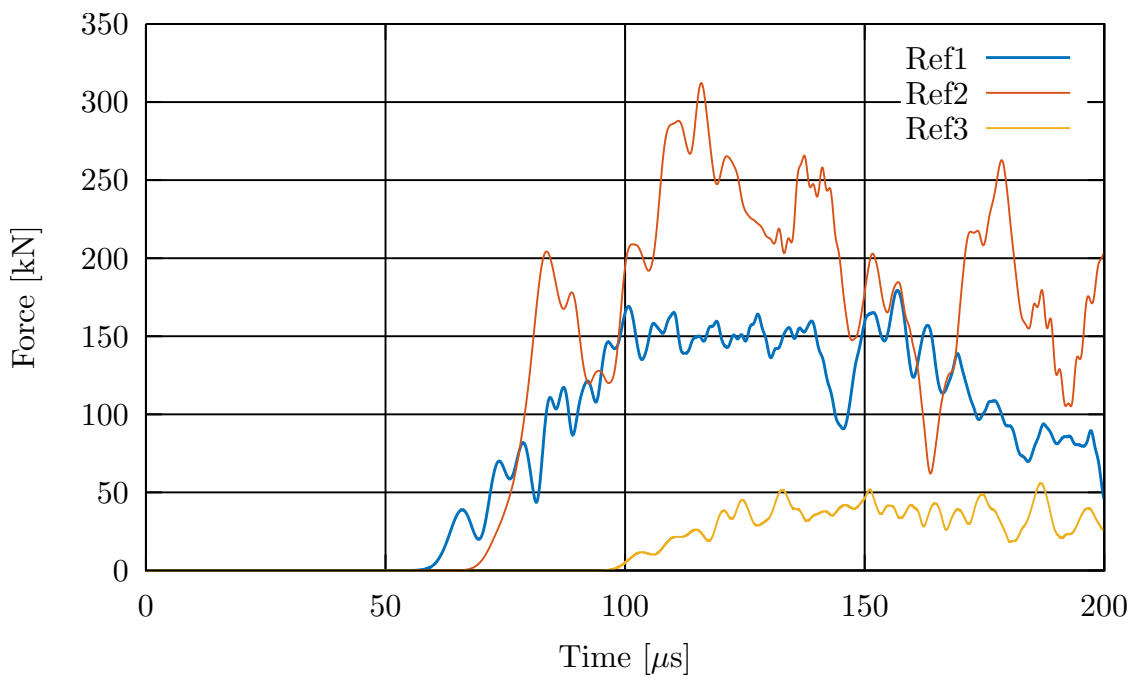

FIGURE 4.8: Force modulus on one of the constraints Reference 1, Reference 2 and Reference 3.
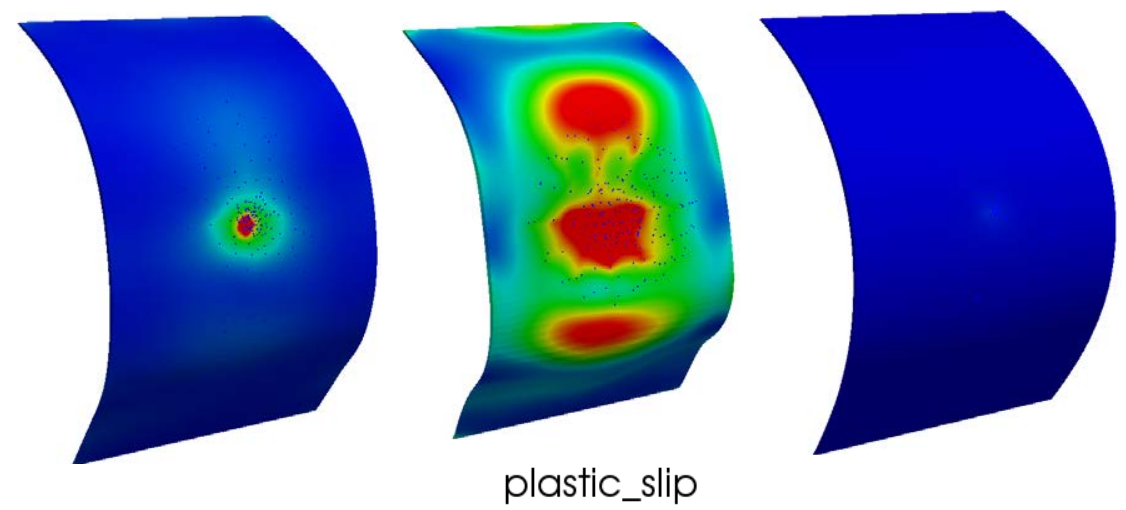

$0.000 e+00$

$1 e-5$

$2.000 \mathrm{e}-05$

FIGURE 4.9: Plastic strain comparison, from left to right Reference 1, Reference 2 and Reference 3. 


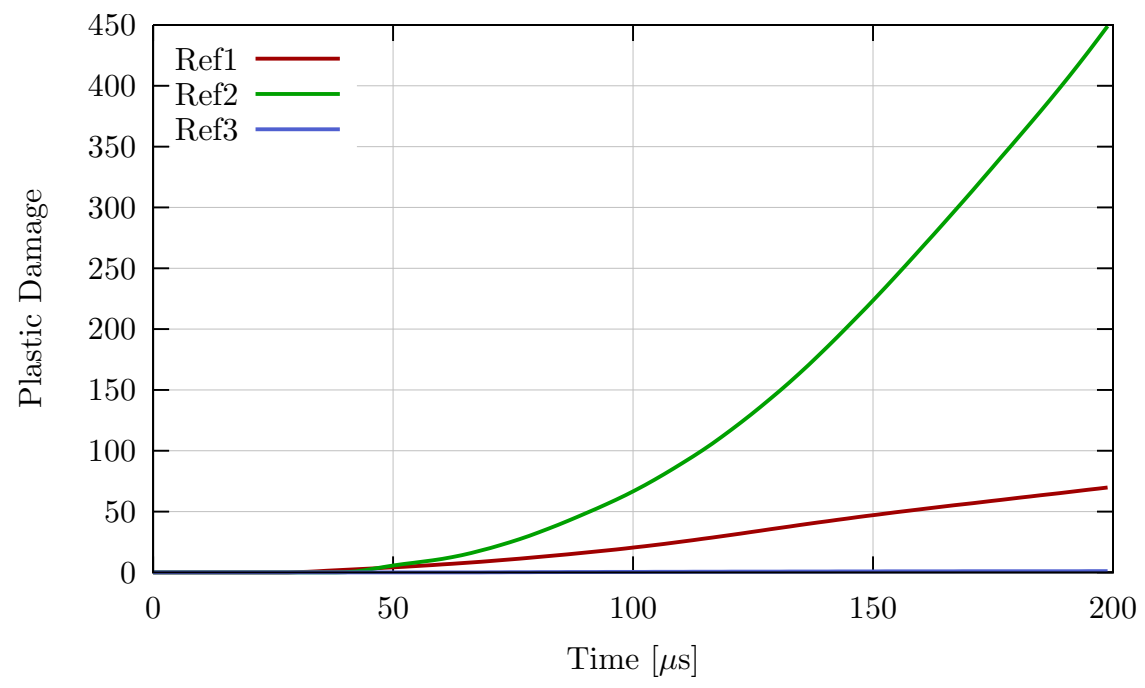

FIGURE 4.10: Plastic damage comparison.

\subsubsection{Projectile speed}

As in Section 4.1.1, velocity increases ice inertia, we expect again an increase in the loads to which the leading edge is subjected, with the increase of velocity. This analysis includes Reference 1, Reference 4 and Reference 5.

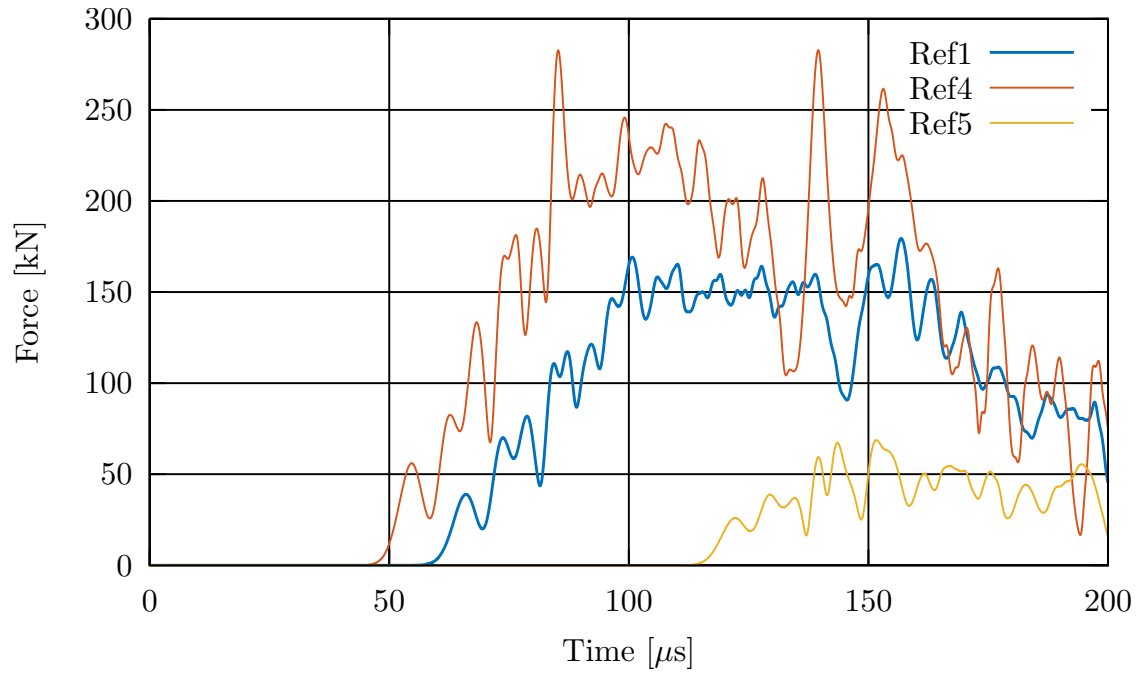

FIGURE 4.11: Force modulus on one of the constraints Reference 1, Reference 4 and Reference 5. 


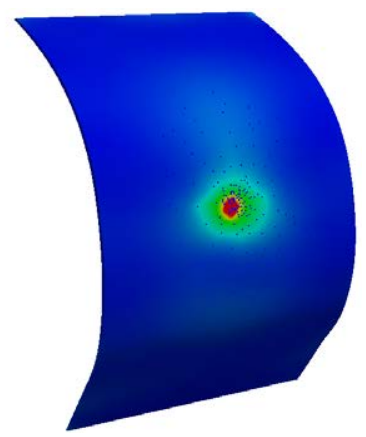

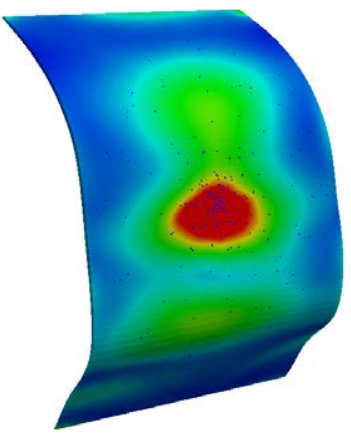

plastic_slip
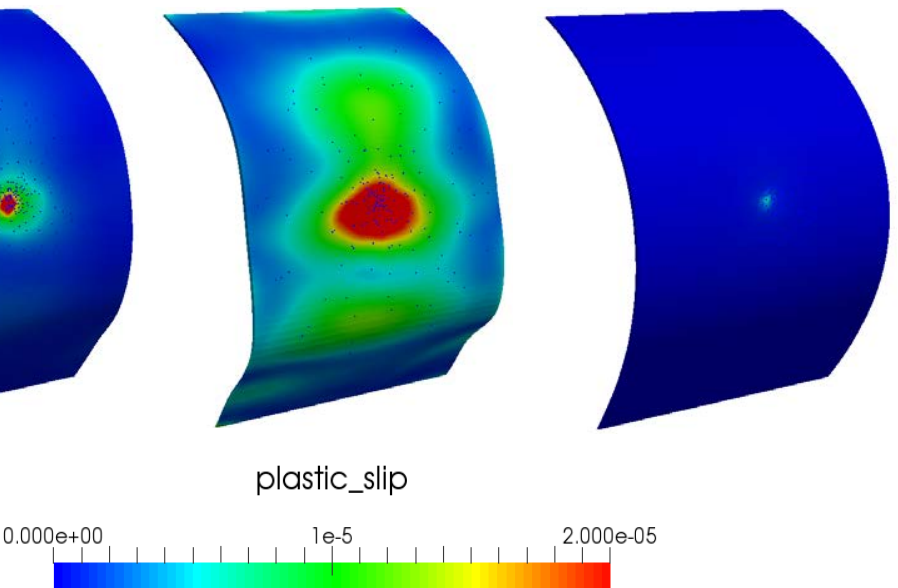

FIGURE 4.12: Plastic strain comparison, from left to right Reference 1, Reference 4 and Reference 5.

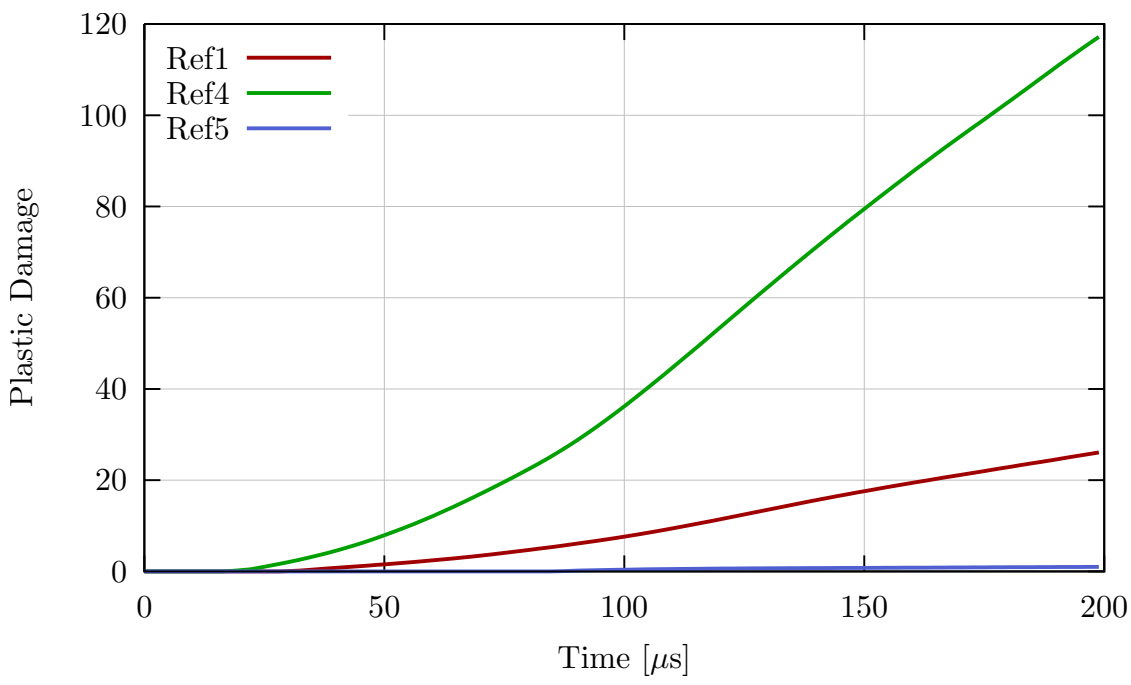

FIGURE 4.13: Plastic damage comparison.

\subsubsection{Projectile strength}

When the ice undergoes deformation due to impact, the cohesion within the material plays an important role, since during impact high pressures are attained at the impact spot, if cohesion fails then the particles can move radially, perpendicular to the impact direction, creating a release wave that reduces the pressure levels which results in the attenuation of loads applied to the leading edge. Thus we expect to have less damage when the projectile strength is reduced. This analysis needs the comparison of Reference 1, Reference 6 and Reference 7. 


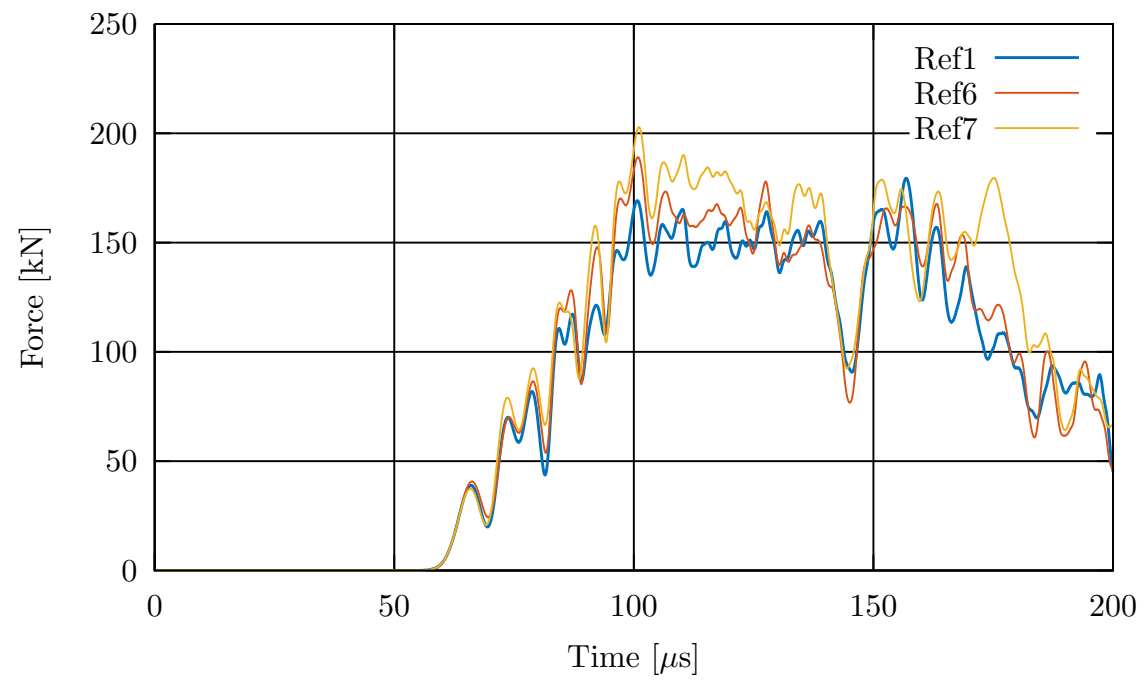

FIGURE 4.14: Force modulus on one of the constraints Reference 1, Reference 6 and Reference 7.
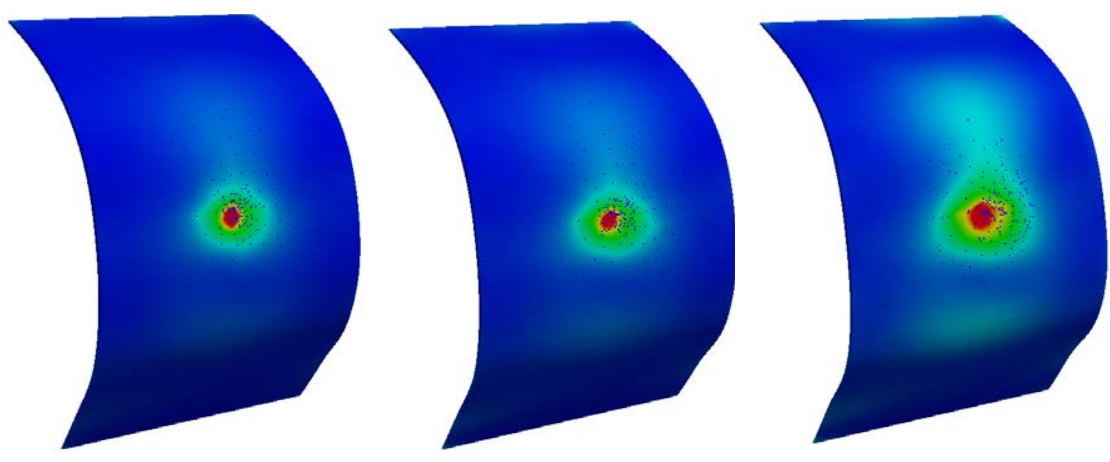

plastic_slip

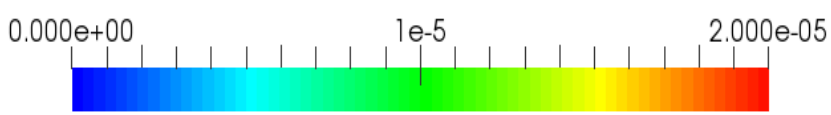

FIGURE 4.15: Plastic strain comparison, from left to right Reference 1, Reference 6 and Reference 7. 


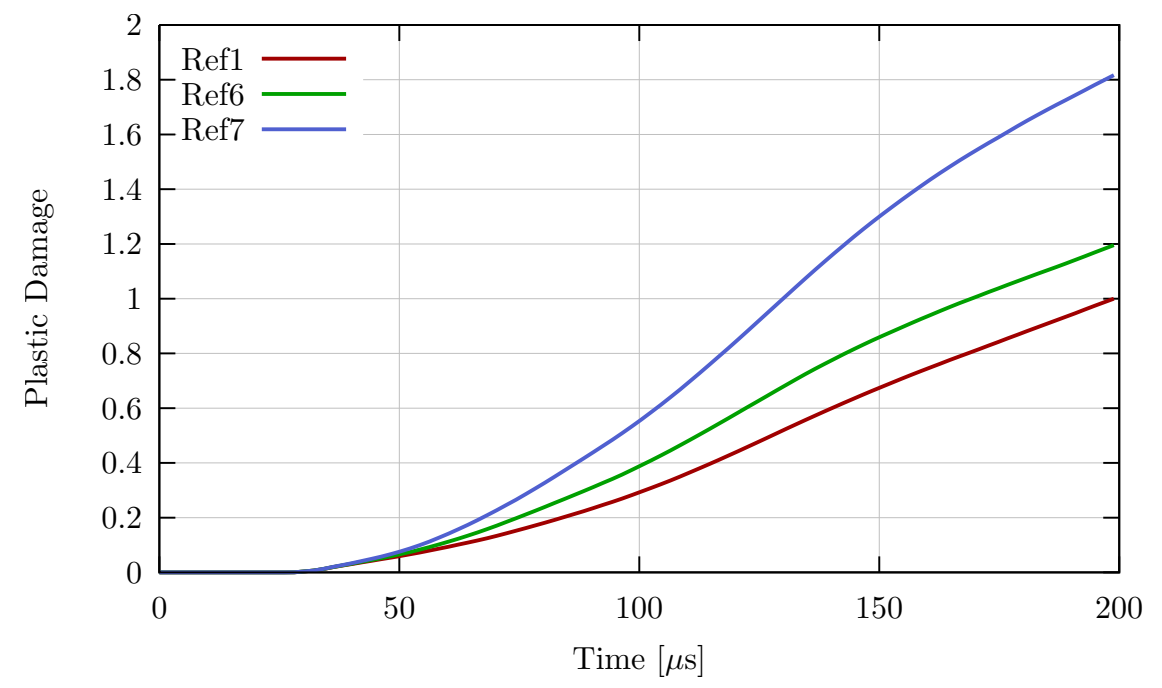

FIGURE 4.16: Plastic damage comparison.

\subsubsection{Projectile impact position}

This analysis will let us to know if there are weak spots in the leading edge, since asymmetric impact will make the loads generated to be worse distributed along the frame, being in theory the best impact position from the point of view of damage, the position in Reference 1. The tests included in this analysis are Reference 1, Reference 8 and Reference 9.

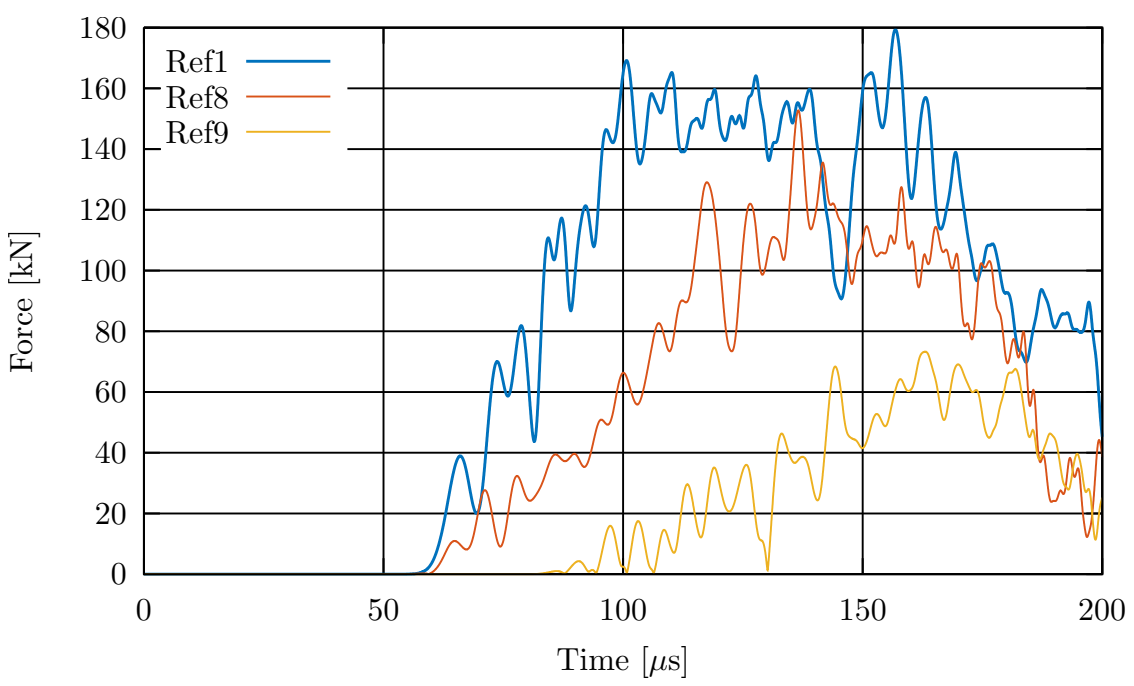

FIGURE 4.17: Force modulus on one of the constraints Reference 1, Reference 8 and Reference 9. 

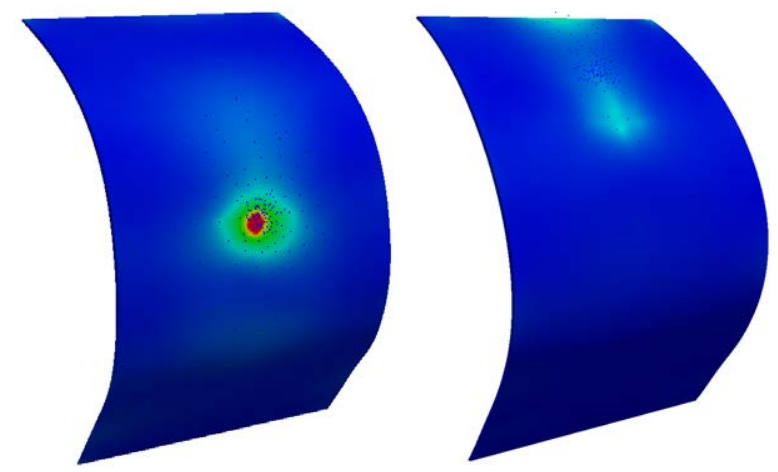

plastic_slip

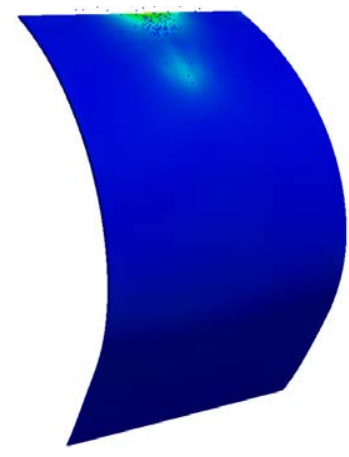

2.000 e-05

FIGURE 4.18: Plastic strain comparison, from left to right Reference

1, Reference 8 and Reference 9.

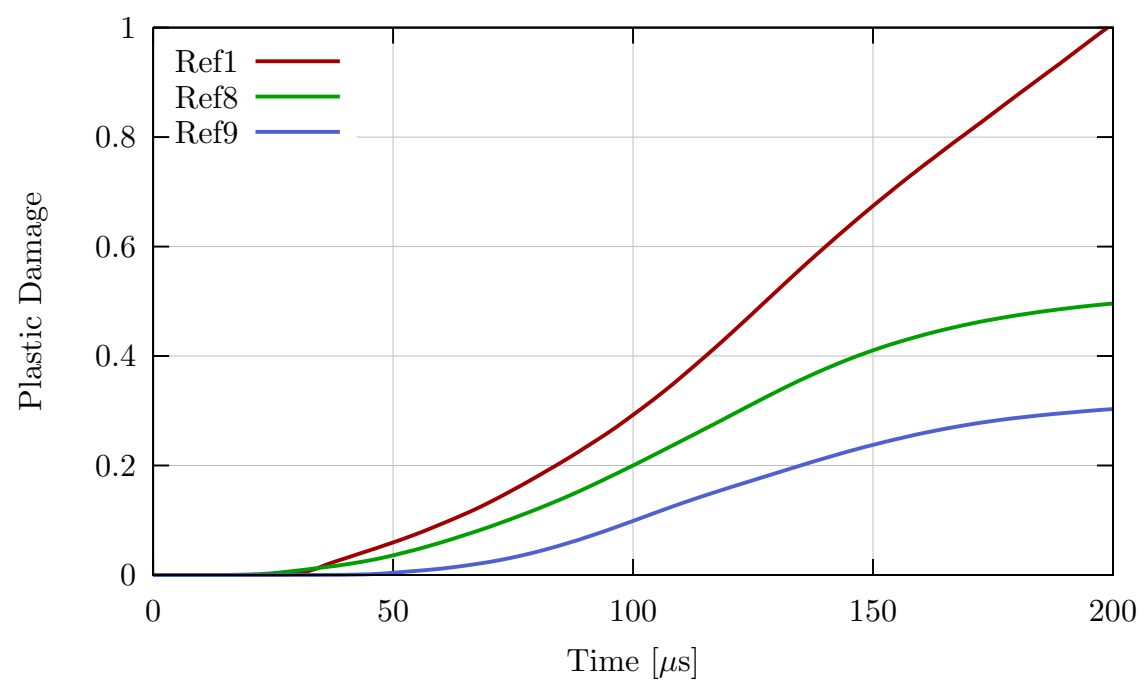

FIGURE 4.19: Plastic damage comparison.

\subsection{Concluding remarks}

What we can conclude from Sections 4.1.1, 4.1.2, 4.1.3, 4.1.4 and in view of Fig 4.10 is that there is an important influence of the mass with respect to the damage produced. We observe that by doubling the size of the sphere, which results in and increase of mass of 8 times the reference one, yields an increase of the plastic damage in one order of magnitude. Secondly, from Fig. 4.13 as a rough estimation one could claim that doubling the velocity will increase the resulted plastic damage by one order of magnitude. However, the projectile strength barely influences the resulted plastic damage as shown in Fig. 4.16 for the given material parameters of ice, see Table 4.2, doubling the pressure cut-offs, $p^{+}$and $p^{-}$, will not even double the resulted damage. Finally, the influence of the position clearly shows that the framework is weaker when facing impacts frontally than obliquely. This can be explained from the fact that oblique impacts can be deflected in an easier way reducing the amount of linear momenta exchange between the framework and the ice sphere. 


\section{Chapter 5}

\section{Summary and Conclusions}

The main issue that motivated this work, FOD is still nowadays a threat to aeronautics and passengers safety as we have witnessed recently, in flight 1897 American Airlines, the 3rd of June 2018. Improvement in strength or design of the structural parts of the aircraft needs to be achieved. This means to conduct real tests that recreate the conditions of FOD events. These experimental set-ups are expensive and limited to the number of conditions they can reproduce. The other option is then simulation. Having robust numerical frameworks will allow engineers to try as many impact conditions as needed, assessing their designs and improving them.

In order to achieve a robust framework for high speed impact simulation, we identified, first, available numerical tools used for this purpose and assessed their weaknesses. Whenever we pinpointed ways of improving the existing numerical tools, we tried to make an step forward. The things that we identified that were amenable to improvement were:

- The formulation of viscoplasticity.

- The contact algorithm.

- Failure criteria for brittle materials.

- Meshless discretisations.

We reviewed the existing knowledge for constitutive models that account for rate effects and irreversible deformation, the so-called elasto-viscoplastic models. The formulations of Perzyna and Duvaut-Lions were identified as the proper choice for modelling this behaviour due to the nature of their formulation that ensures nonnegative dissipation. From the cornerstone of the second law of thermodynamics and generalising Duvaut-Lions viscoplasticity we developed a general class of integration algorithms for rate-dependent elasto-viscoplasticity. Their most significant features are that they are applicable for material models based on non-smooth yield functions, including hardening, and for fairly general viscoplastic flow rules. We have been able to define very general expressions that can be used to model viscoplastic effects with great flexibility, yet strictly satisfying a discrete dissipation inequality that mimics the behavior of the continuum models. These methods can be used to integrate viscoplastic models based on smooth and non-smooth yield criteria, with or without isotropic and kinematic hardening. For these concepts to hold, the models are restricted to linear hardening, a limitation that can only be overcome at the expense of losing the simplicity of the solution. We presented the full details of the numerical schemes required to integrate viscoplastic models based on Tresca's and Druker-Prager's yield functions, with general (convex) kinetic potentials. These methods are developed in small strain theory, however they can be easily extensible to accomodate large strain theory with the logarithmic measure. In summary, as a 
generalisation of Duvaut-Lion model, we have obtained a general class of viscoplasticity for any type of plasticity criteria and a wide variety of different rate effect laws that ensures non-negative dissipation and enriches the material behaviours to wich this model can be adapted.

Contact is a key point in this work, since impact involves interaction between bodies. However, the existing contact algorithms in explicit schemes based on penalty methods were deemed to be not appropriate. They do not ensure impenetrability and impose restriction on time step that might affect stability or extend to an unaffordable computing time the duration of the simulation. The predictor-corrector contact algorithms do not suffer from that. Works with this implementation were analysed to finally led us to the formulation of a new algorithm for contact/impact in explicit simulations of deformable bodies. The proposed method has several salient features that make it very appealing for general purpose computations. First, it imposes very mild stability restrictions on the time step size employed in the integration. Second, it bypasses the need to choose a penalty parameter since the impenetrability constraint is not imposed with an artificial stiffness, but rather with a projection scheme. Third, it possesses favorable energy estimates for contact with or without friction and/or perfect restitution. Fourth, it is asymmetric by construction, and thus can be employed for modeling interactions between finite element models, or between a finite element model and a meshfree one. Overall, the method is simple to implement and its foundations are pretty intuitive, it is extremely robust for all types of contact simulations, including those involving bodies with sharp edges or corners. As shown, it correctly reproduces analytic solutions of reference. Thus we have been able to develop a new contact algorithm for explicit dynamics that has a better performance than those based on penalty restrictions and can be extended for interactions of any type of spatial discretisation.

Failure criteria is an important point for the impact of low strength materials such as bird and ice, since cohesion fails in the primary stages of impact. This work succinctly reviewed the available options. Yet since their implementation is pretty straightforward, they are briefly introduced with a simple case that is able to reproduce the late stages of the crushed ice as seen in Chapter 4.

During the development of this work, since part of the initial studies were done in LS-DYNA, we identified the limited accessibility and amenability to support user defined material subroutines in commercial codes. To the authors knowledge this issues have never been resolved, becoming a possible tool to develop that might be in the general interest of the computational mechanics community. The library MUESLI is designed to be used with research and commercial codes with the purpose of providing an accessible tool that could become a common standard for the development of material modelling.

All the simulations performed for this work, including contact, Max-Ent, FEM, etc. were done in IRIS, an in-house research code developed at the Computational Mechanics group in IMDEA Materials.

After a thorough review of the available spatial discretisation methods for severely distorted discretised bodies and soon discarding those that rely on a mesh like FEM, we concluded that meshless methods with the Kronecker-delta property are the most recommended. We mentioned those that rely on convex approximation schemes like NEM and Max-ent. Finally we opted for Max-ent, which has more convenient features. However, this iterative method might encounter issues with convergence for pathological nodal distributions.

This set of numerical tools constitute a solid framework on which the simulation of high speed impact and mainly the impact of ice and bird can be addressed, with 
properly tunned material parameters to fit their real response, with a contact that faithfully reproduces reality preserving linear momentum and having favorable energy exchanges, we can ensure that the results obtained in the simulation faithfully reproduce those that would be obtained in real tests. This is supported with the simulation performed in Chapter 4 , by using the notion of plastic damage we can compare, to a certain extent, different impact conditions with just one positive scalar, openning wide possibilities for the analysis of FOD and the reduction of its threat in aeronautics.

\section{Main contributions}

This work has given the opportunity of publishing three articles:

- MUESLI-a Material UnivErSal LIbrary, see [59].

- Formulation and numerical solution of non-smooth elasto-visco-plasticity models, see [58].

- A robust asymmetrical contact algorithm for explicit solid dynamics (submitted to Computational Mechanics).

This work has been presented in several congresses:

- del Pozo, D., \& Romero, I. (2017, July). Non-Linear Viscoplasticity for NonSmooth Yield Surfaces. Presented at the Congress on Numerical Methods in Engineering CMN2017 (Vol. 3, p. 5).

- del Pozo, D., \& Romero, I. (2016, May). Non-Linear Viscoplasticity for NonSmooth Yield Surfaces. Presented at the 10th Conference on Mechanics of Time-Dependent Materials MTDM2016, May 17-20 (2016).

- del Pozo, D., López, I., \& Romero, I. (2018, June). Predictor-Corrector Contact Algorithm between Meshless and Finite Elements Methods. Presented at the 6th European Conference on Computational Mechanics (ECCM 6). June 11-15 (2018).

\section{Future work}

Beyond the results written in these pages, we outline now which might be the future lines of research in this field:

- Max-ent method is computationally expensive and might encounter issues in the iterative methods for the minimisation. Ideal meshfree spatial discretisations would have to verify the Kronecker-delta property and being sufficiently smooth, but at the same time easy to compute.

- When cracks appear in a meshless body, the construction of the shape functions that are close to the crack will include in their support nodes that are on the other side of the crack, this means accounting for irrelevant and in fact misleading kinematic information for computing the stress and the update of internal variables of material points, certainly this will spoil the results in that region. The same might happen for intricate non-convex bodies with too close boundaries. A framework for detecting these situations will enlarge the possible conditions that can be simulated. 
- The contact approach presented seems to be a good option, however its implementation up to now has been addressed to accommodate only meshless-FEM interaction. Other options could be investigated, such as meshless-meshless interaction.

- The viscoplastic formulation developed could include non-linear hardening but losing the elegance of the integration scheme, nevertheless alternatives could be investigated to keep the same structure of the integration. 


\section{Appendix A}

\section{Appendices}

\section{A.1 Derivation of consistent tangent tensors}

We provide the details, in this appendix, for the derivation of the consistent tangent of elasticities for the elasto-visco-plastic models with Tresca's and Drucker-Prager's yield functions. These tensors are required, among other things, for the implicit solution of elasto-visco-plastic problems, such as the ones in Sec. 2.4.

First, let us address the general situation, for a model with an arbitrary (convex) yield function. To find the generalized stress $\boldsymbol{\Sigma}_{n+1}$ in Eq. 2.46), let us define the scalar

$$
\omega_{n+1}=\frac{\Delta t}{\tau} \frac{\delta J^{\prime}\left(d_{\mathbb{E}}\left(\boldsymbol{\Sigma}_{n+1}\right) / \delta\right)}{d_{\mathbb{E}}\left(\boldsymbol{\Sigma}_{n+1}\right)},
$$

and rewrite Eq. (2.46) in the compact form

$$
\boldsymbol{\Sigma}_{n+1}=\frac{1}{1+\omega_{n+1}} \boldsymbol{\Sigma}_{n+1}^{t r}+\frac{\omega_{n+1}}{1+\omega_{n+1}} \overline{\boldsymbol{\Sigma}}_{n+1}^{t r} .
$$

Noting that $\boldsymbol{\Sigma}_{n+1}=(\boldsymbol{\sigma}, q)$, we use the previous equation to calculate the consistent tangent tensor $\mathbb{C}_{v p}=\frac{\partial \boldsymbol{\sigma}_{n+1}}{\partial \boldsymbol{\varepsilon}_{n+1}}$. For that, we recall that $\boldsymbol{\Sigma}_{n+1}^{t r}$ is the result of a purely elastic step, and $\overline{\boldsymbol{\Sigma}}_{n+1}^{t r}$, the rate independent solution. Thus,

$$
\mathbb{C}_{e}=\frac{\partial \boldsymbol{\sigma}_{n+1}^{t r}}{\partial \varepsilon_{n+1}}, \quad \mathbb{C}_{e p}=\frac{\partial \overline{\boldsymbol{\sigma}}_{n+1}^{t r}}{\partial \varepsilon_{n+1}}
$$

are the elastic and elasto-plastic tangent tensors, respectively. Using these results, we can express the consistent tangent as

$$
\begin{aligned}
\mathbb{C}_{v p}= & \frac{1}{1+\omega_{n+1}} \mathbb{C}_{e}+\boldsymbol{\sigma}_{n+1}^{t r} \otimes \frac{\partial}{\partial \varepsilon_{n+1}}\left(\frac{1}{1+\omega_{n+1}}\right) \\
& +\frac{\omega_{n+1}}{1+\omega_{n+1}} \mathbb{C}_{e p}+\overline{\boldsymbol{\sigma}}_{n+1} \otimes \frac{\partial}{\partial \varepsilon_{n+1}}\left(\frac{\omega_{n+1}}{1+\omega_{n+1}}\right) .
\end{aligned}
$$

The computation of the derivatives of $\omega_{n+1}$ in the previous equation requires the derivative of the distance to the elastic set $d_{\mathbb{E}}\left(\boldsymbol{\Sigma}_{n+1}\right)$ with respect to the strain. Since the distance itself includes in its definition the stress state $\boldsymbol{\sigma}_{n+1}$, we are led to an implicit expression for the viscoplastic tangent. Using Eq. (2.45) and a straightforward manipulation, the derivative of $d_{\mathbb{E}}\left(\boldsymbol{\Sigma}_{n+1}\right)$ can be found to be, when the plasticity 


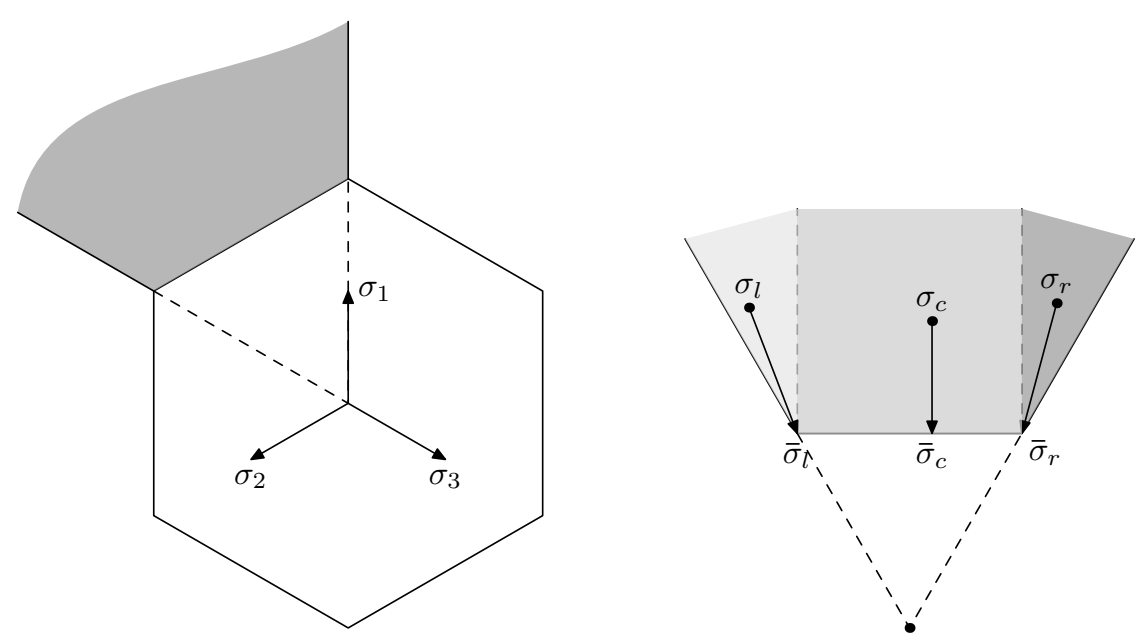

FIGURE A.1: On the left, cross section of the yield surface of Tresca's model in principal stress space, as viewed from the hydrostatic axis, and identification of one of the six sextants that partition the space. On the right, on a generic sextant, the three possible projections of the stress onto the yield surface (left vertex, center plane, and right vertex). For simplicity, no isotropic hardening is considered.

model only has linear isotropic hardening, of the form

$$
\begin{aligned}
\frac{\partial d_{\mathbb{E}}\left(\boldsymbol{\Sigma}_{n+1}^{t r}\right)}{\partial \varepsilon_{n+1}}= & \frac{\delta}{d_{\mathbb{E}}\left(\boldsymbol{\Sigma}_{n+1}^{t r}\right)}\left(\left(\mathbb{I}^{s}-\mathbb{C}_{e p}: \mathbb{C}_{e}^{-1}\right):\left(\boldsymbol{\sigma}_{n+1}^{t r}-\overline{\boldsymbol{\sigma}}_{n+1}\right)\right) \\
& -\frac{\delta}{d_{\mathbb{E}}\left(\boldsymbol{\Sigma}_{n+1}^{t r}\right)}\left(H_{i s o}^{-1}\left(q_{n}-\bar{q}_{n+1}\right) \frac{\partial \bar{q}_{n+1}}{\partial \boldsymbol{\varepsilon}_{n+1}}\right),
\end{aligned}
$$

where $\mathbb{I}^{s}$ is the fourth-order identity tensor on symmetric tensors, and $q_{n}, \bar{q}_{n+1}$ are the conjugate forces of the strain-like isotropic hardening at the trial state (plastic update frozen) and the rate independent solution (projection), respectively.

Expression (A.5) is valid for very general elasto-visco-plastic constitutive models, depending only on objects that are known either from the projection or the rate independent steps. In particular, the derivative $\frac{\partial \bar{q}_{n+1}}{\partial \varepsilon_{n+1}}$ can always be obtained from the inviscid solution.

\section{A.1.1 Tresca}

To compute the derivative $\frac{\partial \bar{q}_{n+1}}{\partial \varepsilon_{n+1}}$ for Tresca's model, we take advantage of the symmetries of the elastic domain and partition the space of principal stresses in six sextants, as depicted in Fig. A.1. By considering only the sextant to which the trial stress belongs to, the inviscid solution can be obtained as the projection onto the smooth part of the surface, or to either the left or right edge. See again Fig. A.1. For each of the cases we wave

1. Stress projected onto smooth region:

$$
\frac{\partial \bar{q}_{n+1}}{\partial \varepsilon_{n+1}}=-\frac{2 \mu H_{i s o}}{4 \mu+H_{i s o}}\left(\frac{\partial \varepsilon_{d 1}^{t r}}{\partial\left(\operatorname{dev}\left(\varepsilon_{n+1}^{t r}\right)\right)}-\frac{\partial \varepsilon_{d 3}^{t r}}{\partial\left(\operatorname{dev}\left(\varepsilon_{n+1}^{t r}\right)\right)}\right) \cdot \mathbb{I}^{d e v},
$$


2. Stress projected onto the right edge:

$$
\begin{aligned}
\frac{\partial \bar{q}_{n+1}}{\partial \varepsilon_{n+1}} & =\frac{2 \mu H_{i s o}}{\operatorname{det}(\boldsymbol{d})}\left(-4 \mu \frac{\partial \varepsilon_{d 1}^{t r}}{\partial\left(\operatorname{dev}\left(\varepsilon_{n+1}^{t r}\right)\right)}\right) \cdot \mathbb{I}^{\text {dev }} \\
& +\frac{2 \mu H_{i s o}}{\operatorname{det}(\boldsymbol{d})}\left(2 \mu \frac{\partial \varepsilon_{d 2}^{t r}}{\partial\left(\operatorname{dev}\left(\varepsilon_{n+1}^{t r}\right)\right)}+2 \mu \frac{\partial \varepsilon_{d 3}^{t r}}{\partial\left(\operatorname{dev}\left(\varepsilon_{n+1}^{t r}\right)\right)}\right) \cdot \mathbb{I}^{\text {dev }},
\end{aligned}
$$

where we have introduced the matrix

$$
\boldsymbol{d}=\left[\begin{array}{ll}
-4 \mu-H_{\text {iso }} & -2 \mu-H_{\text {iso }} \\
-2 \mu-H_{\text {iso }} & -4 \mu-H_{\text {iso }}
\end{array}\right]
$$

3. Stress projected onto the left edge:

$$
\begin{aligned}
\frac{\partial \bar{q}_{n+1}}{\partial \varepsilon_{n+1}} & =\frac{2 \mu H_{i s o}}{\operatorname{det}(\boldsymbol{d})}\left(-2 \mu \frac{\partial \varepsilon_{d 1}^{t r}}{\partial\left(\operatorname{dev}\left(\varepsilon_{n+1}^{t r}\right)\right)}\right) \cdot \mathbb{I}^{d e v} \\
& +\frac{2 \mu H_{i s o}}{\operatorname{det}(\boldsymbol{d})}\left(-2 \mu \frac{\partial \varepsilon_{d 2}^{t r}}{\partial\left(\operatorname{dev}\left(\varepsilon_{n+1}^{t r}\right)\right)}+4 \mu \frac{\partial \varepsilon_{d 3}^{t r}}{\partial\left(\operatorname{dev}\left(\varepsilon_{n+1}^{t r}\right)\right)}\right) \cdot \mathbb{I}^{d e v} .
\end{aligned}
$$

In the last expressions, $\frac{\partial \varepsilon_{d i}^{t r}}{\partial \operatorname{dev}\left(\varepsilon_{n+1}^{t r}\right)}$ refers to the derivative of the $i$-th eigenvalue of the deviatoric trial strain tensor with respect to this tensor. The calculation of this expression is done with the expressions of the eigenvalues in closed form, e.g. Eq. A.39 in [42] and the derivatives of the principal invariants of a second order tensor.

\section{A.1.2 Drucker-Prager}

The second model considered in Sec. 2.4 is based on Drucker-Prager's yield function. When considering only linear isotropic hardening, it is straightforward to verify that the derivative $\frac{\partial \bar{q}_{n+1}}{\partial \varepsilon_{n+1}}$ is of the following form:

1. When the trial stress projects onto the smooth part of the yield surface:

$$
\frac{\partial \bar{q}_{n+1}}{\partial \boldsymbol{\varepsilon}_{n+1}}=-\frac{H_{i s o}\left(\alpha+\frac{\sqrt{3}}{3}\right)}{\mu+9 \kappa \alpha^{2}+H_{i s o}\left(\alpha+\frac{\sqrt{3}}{3}\right)^{2}}\left(\sqrt{2} \mu \boldsymbol{e}_{n+1}+3 \alpha \kappa \boldsymbol{I}\right),
$$

where $e_{n+1}$ is the deviatoric part of strain tensor $\varepsilon_{n+1}-\varepsilon_{n}^{p}$, divided by its norm.

2. When the trial stress projects to the apex of the yield surface:

$$
\frac{\partial \bar{q}_{n+1}}{\partial \varepsilon_{n+1}}=-\frac{3 \alpha H_{i s o} \kappa\left(\alpha+\frac{\sqrt{3}}{3}\right)}{9 \kappa \alpha^{2}+H_{i s o}\left(\alpha+\frac{\sqrt{3}}{3}\right)^{2}} \boldsymbol{I}
$$





\section{Bibliography}

[1] Wikipedia, Foreign object damage - wikipedia, the free encyclopedia, [Online; accessed 20-April-2015], 2015.

[2] B. Boyce, A Mehta, J. Peters, and R. Ritchie, "Synchrotron mesodiffraction: a tool for understanding turbine engine foreign object damage", 2003.

[3] R. K. Souter and J. B. Emerson, "Summary of available hail literature and the effect of hail on aircraft flight", NACA, technical note 2734, 1952.

[4] S. M and L. JL, "Damage from hail grounds nearly $100 \mathrm{~d} / \mathrm{fw}$ planes passengers scramble as scores of flights cancelled", Dallas Morning News, 1 May 1995. p. 1A.

[5] E. M. Schulson, "Brittle failure of ice", Engineering Fracture Mechanics, vol. 68, pp. 1839-1887, 2001.

[6] _ " "The structure and mechanical behavior of ice", The Journal of The Minerals, Metals and Materials Society, vol. 51, pp. 21-27, 21999.

[7] E. M. Schulson and P. Duval, Creep and Fracture of Ice. Cambridge University Press, 2009.

[8] J. Petrovic, "Review mechanical properties of ice and snow", Journal of Materials Science, vol. 38, no. 1, pp. 1-6, 2003.

[9] M. Mellor and D. M. Cole, "Deformation and failure of ice under constant stress or constant strain-rate", Cold Regions Science and Technology, vol. 5, no. 3, pp. 201-219, 1982.

[10] M. Rist and S. Murrell, "Ice triaxial deformation and fracture", Journal of Glaciology, vol. 40, pp. 305-318, 1994.

[11] M. Shazly, V. Prakash, and B. A. Lerch, "High strain-rate behavior of ice under uniaxial compression", International Journal of Solids and Structures, vol. 46, no. 6, pp. 1499-1515, 2009.

[12] S. J. Jones, "High strain-rate compression tests on ice", The Journal of Physical Chemistry B, vol. 101, no. 32, pp. 6099-6101, 1997.

[13] P. Guégan, R. Othman, D. Lebreton, F. Pasco, P. Villedieu, J. Meyssonnier, and S. Wintenberger, "Experimental investigation of the kinematics of postimpact ice fragments", International Journal of Impact Engineering, vol. 38, no. 10, pp. 786-795, 2011.

[14] A. Combescure, Y. Chuzel-Marmot, and J. Fabis, "Experimental study of highvelocity impact and fracture of ice", International Journal of Solids and Structures, vol. 48, no. 20, pp. 2779-2790, 2011.

[15] A. Derradji-Aouat, "Multi-surface failure criterion for saline ice in the brittle regime", Cold Regions Science and Technology, vol. 36, no. 1, pp. 47-70, 2003.

[16] Reduce your risk of bird, wildlife collisions, https://www.nbaa.org/ops/safety/inflight-safety/wildlife-strike/, 2013. 
[17] S. Heimbs, "Computational methods for bird strike simulations: a review", Computers \& Structures, vol. 89, no. 23-24, pp. 2093-2112, 2011.

[18] S. Meguid, R. Mao, and T. Ng, "Fe analysis of geometry effects of an artificial bird striking an aeroengine fan blade", International Journal of Impact Engineering, vol. 35, no. 6, pp. 487-498, 2008.

[19] J. S. Wilbeck, "Impact behavior of low strength projectiles", Air Force Materials Lab Wright-Patterson AFB OH, Tech. Rep., 1978.

[20] M. Lavoie, A Gakwaya, M. N. Ensan, D. Zimcik, and D Nandlall, "Bird's substitute tests results and evaluation of available numerical methods", International Journal of Impact Engineering, vol. 36, no. 10-11, pp. 1276-1287, 2009.

[21] R. Hedayati and M. Sadighi, Bird Strike: An Experimental, Theoretical and Numerical Investigation. Woodhead Publishing, 2015.

[22] B. Luccioni and G. Aráoz, "Mecánica computacional, volume xxx. number 21. modeling of materials failure (a)",

[23] A. Pandolfi and M. Ortiz, "An eigenerosion approach to brittle fracture", International Journal for Numerical Methods in Engineering, vol. 92, no. 8, pp. 694714, 2012.

[24] J. O. Hallquist, LS-DYNA Theoretical Manual. Livermore Software Technology Corporation, 1998.

[25] M. Anghileri, Luigi-M.L.Castelletti, F. Invernizzi, and M. Mascheroni, "A survey of numerical models for hail impact analysis using explicit finite element codes", International Journal of Impact Engineering, vol. 31, pp. 929-944, 2005.

[26] H. Kim and K. T. Kedward, "Modeling hail ice impacts and predicting impact damage initiation in composite structures", AIAA Journal, vol. 38, no. 7, 2000.

[27] H. Park and H. Kim, "Damage resistance of single lap adhesive composite joints by transverse ice impact", International Journal of Impact Engineering, vol. 37, no. 2, pp. 177-184, 2010.

[28] K. S. Carney, D. J.Benson, P. du Bois, and R. Lee, "A phenomenological high strain rate model with failure for ice", International Journal of Solids and Structures, vol. 43, pp. 7820-7839, 2006.

[29] — " "A high strain rate model with failure for ice in LS-DYNA", in 9th International LS-DYNA Users Conference.

[30] T. Sain and R.Narasimhan, "Constitutive modeling of ice in the high strain rate regime", International Journal of Solids and Structures, vol. 48, pp. 817-827, 2011.

[31] A Airoldi and B Cacchione, "Modelling of impact forces and pressures in lagrangian bird strike analyses", International Journal of Impact Engineering, vol. 32, no. 10, pp. 1651-1677, 2006.

[32] J. Pernas-Sánchez, D. A. Pedroche, D. Varas, J. López-Puente, and R. Zaera, "Numerical modeling of ice behavior under high velocity impacts", International Journal of Solids and Structures, vol. 49, no. 14, pp. 1919-1927, 2012.

[33] A. Hanssen, Y Girard, L Olovsson, T Berstad, and M Langseth, "A numerical model for bird strike of aluminium foam-based sandwich panels", International Journal of Impact Engineering, vol. 32, no. 7, pp. 1127-1144, 2006.

[34] L. B. Lucy, "A numerical approach to the testing of the fission hypothesis", The Astronomical Journal, vol. 82, pp. 1013-1024, 1977. 
[35] R. A. Gingold and J. J. Monaghan, "Smoothed particle hydrodynamics: theory and application to non-spherical stars", Monthly Notices of the Royal Astronomical Society, vol. 181, no. 3, pp. 375-389, 1977.

[36] Y. Chuzel, “Caractérisation expérimentale et simulation numérique d'impacts de glace à haute vitesse", PhD thesis, Villeurbanne, INSA, 2009.

[37] T Belytschko and M. O. Neal, "Contact-impact by the pinball algorithm with penalty and Lagrangian methods", International Journal for Numerical Methods in Engineering, vol. 31, pp. 547-572, 1991.

[38] M. A. Lavoie, M. N. Ensan, and A. Gakwaya, "Development of an efficient numerical model for hail impact simulation based on experimental data obtained from pressure sensitive film", Mechanics Research Comunications, vol. 38, pp. 72-76, 2011.

[39] M Guida, F Marulo, M. Meo, A Grimaldi, and G. Olivares, "Sph-lagrangian study of bird impact on leading edge wing", Composite Structures, vol. 93, no. 3, pp. 1060-1071, 2011.

[40] B Li, L Perotti, M Adams, J Mihaly, A. Rosakis, M Stalzer, and M. Ortiz, "Large scale optimal transportation meshfree (otm) simulations of hypervelocity impact", Procedia Engineering, vol. 58, pp. 320-327, 2013.

[41] B. Li, The optimal transportation method in solid mechanics. California Institute of Technology, 2009.

[42] E. A. de Souza Neto, D. Peric, and D. R. Owen, Computational methods for plasticity: theory and applications. John Wiley \& Sons, 2011.

[43] T. J. Hughes and R. L. Taylor, "Unconditionally stable algorithms for quasistatic elasto/visco-plastic finite element analysis", Computers $\mathcal{E}$ Structures, vol. 8, no. 2, pp. 169-173, 1978.

[44] J. C. Simo and T. J. Hughes, Computational inelasticity. Springer Science \& Business Media, 2006, vol. 7.

[45] O. Zienkiewicz and I. Cormeau, "Visco-plasticity-plasticity and creep in elastic solids-a unified numerical solution approach", International Journal for Numerical Methods in Engineering, vol. 8, no. 4, pp. 821-845, 1974.

[46] P. Perzyna, "Fundamental problems in viscoplasticity", Advances in Applied Mechanics, vol. 9, pp. 243-377, 1966.

[47] J. C. Simo, J.-W. Ju, K. S. Pister, and R. L. Taylor, “Assessment of cap model: consistent return algorithms and rate-dependent extension", Journal of Engineering Mechanics, vol. 114, no. 2, pp. 191-218, 1988.

[48] J. Simo, J. Kennedy, and S Govindjee, "Non-smooth multisurface plasticity and viscoplasticity. loading/unloading conditions and numerical algorithms", International Journal for Numerical Methods in Engineering, vol. 26, no. 10, pp. 2161-2185, 1988.

[49] G. Duvant and J. L. Lions, Inequalities in mechanics and physics. Springer Science \& Business Media, 2012, vol. 219.

[50] J. Ju, "Consistent tangent moduli for a class of viscoplasticity", Journal of Engineering Mechanics, vol. 116, no. 8, pp. 1764-1779, 1990.

[51] J. C. Simo and R. L. Taylor, "Consistent tangent operators for rate-independent elastoplasticity", Computer Methods in Applied Mechanics and Engineering, vol. 48, no. 1, pp. 101-118, 1985. 
[52] W. Han and B. D. Reddy, Plasticity: mathematical theory and numerical analysis. Springer Science \& Business Media, 2012, vol. 9.

[53] F. H. Clarke, Optimization and nonsmooth analysis. SIAM, 1990.

[54] B. Loret and J. H. Prevost, "Accurate numerical solutions for drucker-prager elastic-plastic models", Computer Methods in Applied Mechanics and Engineering, vol. 54, no. 3, pp. 259-277, 1986.

[55] T. J. Hughes, The finite element method: linear static and dynamic finite element analysis. Prentice Hall, 2012.

[56] R. Hill, The mathematical theory of plasticity. Oxford university press, 1998, vol. 11.

[57] A Cuitino and M Ortiz, "A material-independent method for extending stress update algorithms from small-strain plasticity to finite plasticity with multiplicative kinematics", Engineering Computations, vol. 9, no. 4, pp. 437-451, 1992.

[58] D del Pozo and I Romero, "Formulation and numerical solution of non-smooth elasto-visco-plasticity models", Computer Methods in Applied Mechanics and Engineering, vol. 324, pp. 457-475, 2017.

[59] D. Portillo, D. del Pozo, D. Rodríguez-Galán, J. Segurado, and I. Romero, "Muesli-a material universal library", Advances in Engineering Software, vol. 105, pp. 1-8, 2017.

[60] B Nayroles, G Touzot, and P Villon, "Generalizing the finite element method: diffuse approximation and diffuse elements", Computational Mechanics, vol. 10, no. 5, pp. 307-318, 1992.

[61] T. Belytschko, Y. Y. Lu, and L. Gu, "Element-free galerkin methods", International Journal for Numerical Methods in Engineering, vol. 37, no. 2, pp. 229-256, 1994.

[62] W. K. Liu and Y. Chen, "Wavelet and multiple scale reproducing kernel methods", International Journal for Numerical Methods in Fluids, vol. 21, no. 10, pp. 901931, 1995.

[63] W. K. Liu, S. Jun, and Y. F. Zhang, "Reproducing kernel particle methods", International Journal for Numerical Methods in Fluids, vol. 20, no. 8-9, pp. 10811106, 1995.

[64] S. Li and W. K. Liu, Meshfree particle methods. Springer Science \& Business Media, 2007.

[65] C. A. Duarte and J. T. Oden, "An hp adaptive method using clouds", Computer Methods in Applied Mechanics and Engineering, vol. 139, no. 1-4, pp. 237262, 1996.

[66] J. M. Melenk and I. Babuška, "The partition of unity finite element method: basic theory and applications", Computer Methods in Applied Mechanics and Engineering, vol. 139, no. 1-4, pp. 289-314, 1996.

[67] I. Babuška and J. M. Melenk, "The partition of unity method", International Journal for Numerical Methods in Engineering, vol. 40, no. 4, pp. 727-758, 1997.

[68] N. Sukumar, B. Moran, and T Belytschko, "The natural element method in solid mechanics", International Journal for Numerical Methods in Engineering, vol. 43, no. 5, pp. 839-887, 1998. 
[69] N Sukumar, "Construction of polygonal interpolants: a maximum entropy approach", International Journal for Numerical Methods in Engineering, vol. 61, no. 12, pp. 2159-2181, 2004.

[70] M. Arroyo and M. Ortiz, “Local maximum-entropy approximation schemes: a seamless bridge between finite elements and meshfree methods", International Journal for Numerical Methods in Engineering, vol. 65, no. 13, pp. 21672202, 2006.

[71] C. J. Cyron, M Arroyo, and M. Ortiz, "Smooth, second order, non-negative meshfree approximants selected by maximum entropy", International Journal for Numerical Methods in Engineering, vol. 79, no. 13, pp. 1605-1632, 2009.

[72] E. T. Jaynes, "Information theory and statistical mechanics", Physical Review, vol. 106, no. 4, p. 620, 1957.

[73] G Castellazzi and P Krysl, “Displacement-based finite elements with nodal integration for reissner-mindlin plates", International Journal for Numerical Methods in Engineering, vol. 80, no. 2, pp. 135-162, 2009.

[74] P Krysl and B Zhu, "Locking-free continuum displacement finite elements with nodal integration", International Journal for Numerical Methods in Engineering, vol. 76, no. 7, pp. 1020-1043, 2008.

[75] M. Puso, J. Chen, E Zywicz, and W Elmer, "Meshfree and finite element nodal integration methods", International Journal for Numerical Methods in Engineering, vol. 74, no. 3, pp. 416-446, 2008.

[76] Y. Chen, J. Lee, and A. Eskandarian, Meshless methods in solid mechanics. Springer Science \& Business Media, 2006.

[77] G. Liu, B. B. Kee, and L. Chun, "A stabilized least-squares radial point collocation method (ls-rpcm) for adaptive analysis", Computer Methods in Applied Mechanics and Engineering, vol. 195, no. 37-40, pp. 4843-4861, 2006.

[78] G.-R. Liu, Mesh free methods: moving beyond the finite element method. CRC press, 2002.

[79] E. Cueto and F. Chinesta, "Meshless methods for the simulation of material forming", International Journal of Material Forming, vol. 8, no. 1, pp. 25-43, 2015.

[80] C. A. Duarte and J. Oden, A review of some meshless methods to solve partial differential equations. Texas Institute for Computational and Applied Mathematics Austin, TX, 1995.

[81] T. Belytschko, Y. Krongauz, D. Organ, M. Fleming, and P. Krysl, "Meshless methods: an overview and recent developments", Computer Methods in Applied Mechanics and Engineering, vol. 139, no. 1-4, pp. 3-47, 1996.

[82] J. Swegle, D. Hicks, and S. Attaway, "Smoothed particle hydrodynamics stability analysis", Journal of Computational Physics, vol. 116, no. 1, pp. 123-134, 1995.

[83] J. L. Bentley and J. H. Friedman, "Data structures for range searching", ACM Computing Surveys (CSUR), vol. 11, no. 4, pp. 397-409, 1979.

[84] S. Bardenhagen, J. Brackbill, and D. Sulsky, "The material-point method for granular materials", Computer Methods in Applied Mechanics and Engineering, vol. 187, no. 3-4, pp. 529-541, 2000.

[85] P Wriggers, Computational contact mechanics. Wiley, 2002. 
[86] J. G. Malone and N. L. Johnson, "A parallel finite element contact/impact algorithm for non-linear explicit transient analysis: Part I - The search algorithm and contact mechanics", International Journal for Numerical Methods in Engineering, vol. 37, no. 4, pp. 559-590, 1994.

[87] M. W. Heinstein, F. J. Mello, S. W. Attaway, and T. A. Laursen, "Contactimpact modeling in explicit transient dynamics", Computer Methods in Applied Mechanics and Engineering, vol. 187, pp. 621-640, 2000.

[88] T. Belytschko and M. O. Neal, "Contact-impact by the pinball algorithm with penalty and lagrangian methods", International Journal for Numerical Methods in Engineering, vol. 31, no. 3, pp. 547-572, 1991.

[89] N. J. Carpenter, R. L. Taylor, and M. G. Katona, "Lagrange constraints for transient finite element surface contact", International Journal for Numerical Methods in Engineering, vol. 32, no. 1, pp. 103-128, 1991.

[90] L. Taylor and D. Flanagan, "Pronto 3d: a three-dimensional transient solid dynamics program", Sandia National Labs., Albuquerque, NM (USA), Tech. Rep., 1989.

[91] E. Zywicz and M. A. Puso, "A general conjugate-gradient-based predictorcorrector solver for explicit finite-element contact", International Journal for Numerical Methods in Engineering, vol. 44, no. 4, pp. 439-459, 1999.

[92] M. W. F. Cirak, "Decomposition contact response (DCR) for explicit finite element dynamics", International Journal for Numerical Methods in Engineering, vol. 64 , no. 8, 2005.

[93] Z.-H. Zhong and J. Mackerle, "Static contact problems-a review", Engineering Computations, vol. 9, no. 1, pp. 3-37, 1992.

[94] _ _Contact-Impact Problems: A Review With Bibliography", Applied Mechanics Reviews, vol. 47, no. 2, p. 55, 1994.

[95] N. G. Bourago and V. N. Kukudzhanov, "A review of contact algorithms", Mechanics of Solids, vol. 40, no. 1, pp. 35-71, 2005.

[96] T. A. Laursen, Computational contact and impact mechanics. Springer, 2002.

[97] G. L. Goudreau and J. O. Hallquist, "Recent developments in large-scale finite element lagrangian hydrocode technology", Computer Methods in Applied Mechanics and Engineering, vol. 33, 1982.

[98] J. O. Hallquist, G. L. Goudreau, and D. J. Benson, "Sliding interfaces with contact-impact in large-scale Lagrangian computations", Computer Methods in Applied Mechanics and Engineering, vol. 51, no. 1-3, pp. 107-137, 1985.

[99] P Wriggers, T. V. Van, and E Stein, "Finite element formulation of large deformation impact-contact problems with friction", Computers \& Structures, vol. 37, 1990.

[100] M. W. Heinstein, S. W. Attaway, J. W. Swegle, and F. J. Mello, “A general purpose contact detection algorithm for nonlinear structural analysis codes", Sandia National Lab, Tech. Rep. SAND92-2141, 1992.

[101] J. G. Malone and N. L. Johnson, "A parallel finite element contact/impact algorithm for non-linear explicit transient analysis: Part II - Parallel implementation", International Journal for Numerical Methods in Engineering, vol. 37, no. 4, pp. 591-603, 1994. 
[102] S. W. Attaway, B. A. Hendrickson, S. J. Plimpton, D. R. Gardner, C. T. Vaughan, K. H. Brown, and M. W. Heinstein, "A parallel contact detection algorithm for transient solid dynamics simulations using PRONTO3D", Computational Mechanics, vol. 22, pp. 143-159, 1998.

[103] K Brown, S Attaway, S Plimpton, and B Hendrickson, "Parallel strategies for crash and impact simulations", Computer Methods in Applied Mechanics and Engineering, vol. 184, pp. 375-390, 2000.

[104] G. I. Taylor, "The use of flat-ended projectiles for determining dynamic yield stress i. theoretical considerations", Proc. R. Soc. Lond. A, vol. 194, no. 1038, pp. 289-299, 1948.

[105] A. Whiffin, "The use of flat-ended projectiles for determining dynamic yield stress-ii. tests on various metallic materials", Proc. R. Soc. Lond. A, vol. 194, no. 1038 , pp. 300-322, 1948.

[106] M. Brünig and L. Driemeier, "Numerical simulation of taylor impact tests", International Journal of Plasticity, vol. 23, no. 12, pp. 1979-2003, 2007.

[107] G. R. Johnson, "A constitutive model and data for materials subjected to large strains, high strain rates, and high temperatures", Proc. 7th Inf. Sympo. Ballistics, pp. 541-547, 1983.

[108] G. R. Johnson and W. H. Cook, "Fracture characteristics of three metals subjected to various strains, strain rates, temperatures and pressures", Engineering fracture mechanics, vol. 21, no. 1, pp. 31-48, 1985.

[109] G. R. Johnson and R. A. Stryk, "Symmetric contact and sliding interface algorithms for intense impulsive loading computations", Computer methods in applied mechanics and engineering, vol. 190, no. 35-36, pp. 4531-4549, 2001. 